\title{
Bubbling on boundary submanifolds for the Lin-Ni-Takagi problem at higher critical exponents
}

\author{
Manuel del Pino Fethi Mahmoudi Monica Musso
}

\begin{abstract}
Let $\Omega$ be a bounded domain in $\mathbb{R}^{n}$ with smooth boundary $\partial \Omega$. We consider the equation $d^{2} \Delta u-u+u^{\frac{n-k+2}{n-k-2}}=0$ in $\Omega$, under zero Neumann boundary conditions, where $\Omega$ is open, smooth and bounded and $d$ is a small positive parameter. We assume that there is a $k$-dimensional closed, embedded minimal submanifold $K$ of $\partial \Omega$, which is non-degenerate, and certain weighted average of sectional curvatures of $\partial \Omega$ is positive along $K$. Then we prove the existence of a sequence $d=d_{j} \rightarrow 0$ and a positive solution $u_{d}$ such that

$$
d^{2}\left|\nabla u_{d}\right|^{2} \rightarrow S \delta_{K} \quad \text { as } d \rightarrow 0
$$

in the sense of measures, where $\delta_{K}$ stands for the Dirac measure supported on $K$ and $S$ is a positive constant.
\end{abstract}

Keywords. Critical Sobolev Exponent, Blowing-up Solutions, Nondegenerate minimal submanifolds.

\section{Introduction and statement of main results}

Let $\Omega$ be a bounded, smooth domain in $\mathbb{R}^{n}, \nu$ the outer unit normal to $\partial \Omega$ and $q>1$. The semilinear Neumann elliptic problem

$$
d^{2} \Delta u-u+u^{q}=0 \quad \text { in } \Omega, \quad \frac{\partial u}{\partial \nu}=0 \quad \text { on } \partial \Omega
$$

M. del Pino: Departamento de Ingeniería Matemática and CMM, Universidad de Chile, Casilla 170 Correo 3, Santiago, Chile; e-mail: delpino@dim.uchile.cl

F. Mahmoudi: Departamento de Ingeniería Matemática and CMM, Universidad de Chile, Casilla 170 Correo 3, Santiago, Chile; e-mail: fmahmoudi@dim.uchile.cl

M. Musso: Departamento de Matemática, Pontificia Universidad Catolica de Chile, Avda. Vicuña Mackenna 4860, Macul, Chile; e-mail: mmusso@mat.puc.cl

Mathematics Subject Classification (2010): 35J20; 35J60. 
has been widely considered in the literature for more than 20 years. In $1988 \mathrm{Lin}, \mathrm{Ni}$ and Takagi [27] initiated the study of this problem for small values of $d$, motivated by the shadow system of the Gierer-Meinhardt model of biological pattern formation [20]. In that context $u$ roughly represents the (steady) concentration of an activating chemical of the process, which is thought to diffuse slowly in the region $\Omega$, leaving patterns of high concentration such as small spots or narrow stripes.

When $n=2$ or $q<\frac{n+2}{n-2}$ the problem is subcritical, and a positive least energy solution $u_{d}$ exists by a standard compactness argument. This solution corresponds to a minimizer for the the Raleigh quotient

$$
Q_{d}(u)=\frac{d^{2} \int_{\Omega}|\nabla u|^{2}+\int_{\Omega}|u|^{2}}{\left(\int_{\Omega}|u|^{q+1}\right)^{\frac{2}{q+1}}} .
$$

In the papers $[26,27,39,40]$ the authors described accurately the asymptotic behavior of $u_{d}$ as $d \rightarrow 0$. This function maximizes at exactly one point $p_{d}$ which lies on $\partial \Omega$. The asymptotic location of the point $p_{d}$ gets further characterized as

$$
H_{\partial \Omega}\left(p_{d}\right) \rightarrow \max _{p \in \partial \Omega} H_{\partial \Omega}(p)
$$

where $H_{\partial \Omega}$ denotes the mean curvature of $\partial \Omega$. Moreover, the asymptotic shape of $u_{d}$ is indeed highly concentrated around $p_{d}$ in the form

$$
u_{d}(x) \approx w\left(\frac{\left|x-p_{d}\right|}{d}\right)
$$

where $w(|x|)$ is the unique positive, radially symmetric solution to the problem

$$
\Delta w-w+w^{p}=0 \quad \text { in } \mathbb{R}^{n}, \quad \lim _{|x| \rightarrow \infty} w(x)=0,
$$

which decays exponentially. See also [13] for a short proof.

Construction of single and multiple spike-layer patterns for this problem in the subcritical case has been the object of many studies, see for instance $[6,7,8,10,12,13$, $14,21,22,24,25,29,51]$ and the surveys [36, 37]. In particular, in [51] it was found that whenever one has a non-degenerate critical point $p_{0}$ of the mean curvature $H_{\partial \Omega}(p)$, a solution with a profile of the form (1.3) can be found with $p_{d} \rightarrow p_{0}$.

It is natural to look for solutions to Problem (1.1) that exhibit concentration phenomena as $d \rightarrow 0$ not just at points but on higher dimensional sets.

Given a $k$-dimensional submanifold $\Gamma$ of $\partial \Omega$ and assuming that either $k \geq n-2$ or $q<\frac{n-k+2}{n-k-2}$, the question is whether there exists a solution $u_{d}$ which near $\Gamma$ looks like 


$$
u_{d}(x) \approx w\left(\frac{\operatorname{dist}(x, \Gamma)}{d}\right)
$$

where now $w(|y|)$ denotes the unique positive, radially symmetric solution to the problem

$$
\Delta w-w+w^{p}=0 \quad \text { in } \mathbb{R}^{n-k}, \quad \lim _{|y| \rightarrow \infty} w(|y|)=0 .
$$

In $[31,33,34,35]$, the authors have established the existence of a solution with the profile (1.5) when either $\Gamma=\partial \Omega$ or $\Gamma$ is an embedded closed minimal submanifold of $\partial \Omega$, which is in addition non-degenerate in the sense that its Jacobi operator is non-singular (we recall the exact definitions in the next section). This phenomenon is actually quite subtle compared with concentration at points: existence can only be achieved along a sequence of values $d \rightarrow 0$. The parameter $d$ must actually remain suitably away from certain values where resonance occurs, and the topological type of the solution changes: unlike the point concentration case, the Morse index of these solutions is very large and grows as $d \rightarrow 0$.

It is natural to analyze the critical case $q=\frac{n+2}{n-2}$, namely the problem

$$
d^{2} \Delta u-u+u^{\frac{n+2}{n-2}}=0 \quad \text { in } \Omega, \quad \frac{\partial u}{\partial \nu}=0 \quad \text { on } \partial \Omega
$$

The lack of compactness of Sobolev's embedding makes it harder to apply variational arguments. On the other hand, in [1, 48] it was proven that a non-constant least energy solution $u_{d}$ of (1.6), minimizer of (1.2) exists, provided that $d$ be sufficiently small. The behavior of $u_{d}$ as $d \rightarrow 0$ has been clarified in the subsequent works [4, 38, 43]: as in the subcritical case, $u_{d}$ concentrates, having a unique maximum point $p_{d}$ which lies on $\partial \Omega$ with

$$
H_{\partial \Omega}\left(p_{d}\right) \rightarrow \max _{p \in \partial \Omega} H_{\partial \Omega}(p) .
$$

Pohozaev's identity [41] yields nonexistence of positive solutions to Problem (1.4) when $q=\frac{n+2}{n-2}$, and thus the concentration phenomenon must necessarily be different. Unlike the subcritical case, $u_{d}\left(p_{d}\right) \rightarrow+\infty$ the profile of $u_{d}$ near $p_{d}$ is given, for suitable $\mu_{d} \rightarrow 0$, by

$$
u_{d}(x) \approx d^{\frac{n-2}{2}} w_{\mu_{d}}\left(\left|x-p_{d}\right|\right)
$$

where $w_{\mu}(|x|)$ corresponds to the family of radial positive solutions of

$$
\Delta w+w^{\frac{n+2}{n-2}}=0 \quad \text { in } \mathbb{R}^{n}
$$

namely

$$
w_{\mu}(|x|)=\alpha_{n}\left(\frac{\mu}{\mu^{2}+|x|^{2}}\right)^{\frac{n-2}{2}}, \quad \alpha_{n}=(n(n-2))^{\frac{n-2}{4}}
$$


which up to translations, correspond to all positive solutions of (1.8), see [9]. The precise concentration rates $\mu_{d}$ are dimension dependent, and found in the works [5, 23, 43]. In particular $\mu_{d} \sim d^{2}$ for $n \geq 5$, so that $u_{d}\left(p_{d}\right) \sim d^{-\frac{n-2}{2}}$.

As in the subcritical case, construction and estimates for bubbling solutions to Problem (1.6) have been subjects broadly treated. In addition to the above references we refer the reader to $[2,3,16,18,19,28,30,42,44,45,46,49,50,52]$.

In particular, in [3] it was found that for $n \geq 6$ and a non-degenerate critical point $p_{0}$ of the mean curvature with $H_{\partial \Omega}\left(p_{0}\right)>0$, there exists a solution whose profile near $p_{0}$ is given by

$$
u_{d}(x) \approx d^{\frac{n-2}{2}} w_{\mu_{d}}\left(\left|x-p_{0}\right|\right), \quad \quad \mu_{d}=a_{n} H_{\partial \Omega}\left(p_{0}\right)^{\frac{1}{n-2}} d^{2}
$$

for certain explicit constant $a_{n}>0$. See also $[42,43]$ for the lower dimensional case. The condition of critical point for $H_{\partial \Omega}$ with $H_{\partial \Omega}\left(p_{0}\right)>0$ turns out to be necessary for the boundary bubbling phenomenon to take place, see $[5,23]$.

The concentration phenomenon in the critical scenario is more degenerate than that in the subcritical case, and its features harder to be detected because of the rather subtle role of the scaling parameter $\mu$. The purpose of this paper is to unveil the corresponding analog of a solution like (1.10) for the $k$-dimensional concentration question, in the so far open critical case of the $k$-th critical exponent $q=\frac{n-k+2}{n-k-2}$, namely for the problem

$$
d^{2} \Delta u-u+u^{\frac{n-k+2}{n-k-2}}=0 \quad \text { in } \Omega, \quad \frac{\partial u}{\partial \nu}=0 \quad \text { on } \partial \Omega .
$$

Notice that for the Dirichlet problem solution concentrating along boundary geodesics near the second critical exponent have been considered by del Pino, Musso and Pacard in [17].

Let $K$ be $k$-dimensional embedded submanifold of $\partial \Omega$. Under suitable assumptions we shall find a solution $u_{d}(x)$ which for points $x \in \mathbb{R}^{n}$ near $K$, can be described as

$$
x=p+z, \quad p \in K, \quad|z|=\operatorname{dist}(x, K),
$$

we have

$$
u_{d}(x) \approx d^{\frac{n-k-2}{2}} w_{\mu_{d}}(|z|), \quad \mu_{d}(p)=a_{n-k} \bar{H}(p)^{\frac{1}{n-k-2}} d^{2}
$$

where $w_{\mu}$ now denotes

$$
w_{\mu}(|z|)=\alpha_{n-k}\left(\frac{\mu}{\mu^{2}+|z|^{2}}\right)^{\frac{n-k-2}{2}} .
$$

The form of the quantity $\bar{H}(p)$ is of course not obvious. It turns out to correspond to a weighted average of sectional curvatures of $\partial \Omega$ along $K$, which we shall need to assume positive. To explain what it is we need some notation. 
We denote as customary by $T_{p} \partial \Omega$ the tangent space to $\partial \Omega$ at the point $p$. We consider the shape operator $\mathrm{L}: T_{p} \partial \Omega \rightarrow T_{p} \partial \Omega$ defined as

$$
\mathrm{L}[e]:=-\nabla_{e} \nu(p)
$$

where $\nabla_{e} \nu(p)$ is the directional derivative of the vector field $\nu$ in the direction $e$. Let us consider the orthogonal decomposition

$$
T_{p} \partial \Omega=T_{p} K \oplus N_{p} K
$$

where $N_{p} K$ stands for the normal bundle of $K$. We choose orthonormal bases $\left(e_{a}\right)_{a=1, \ldots, k}$ of $T_{p} K$ and $\left(e_{i}\right)_{i=k+1, \ldots, n-1}$ of $N_{p} K$.

Let us consider the $(n-1) \times(n-1)$ matrix $H(p)$ representative of $\mathrm{L}$ in these bases, namely

$$
H_{\alpha \beta}(p)=e_{\alpha} \cdot \mathrm{L}\left[e_{\beta}\right] .
$$

This matrix also represents the second fundamental form of $\partial \Omega$ at $p$ in this basis. $H_{\alpha \alpha}(p)$ corresponds to the curvature of $\partial \Omega$ in the direction $e_{\alpha}$. By definition, the mean curvature of $\partial \Omega$ at $p$ is given by the trace of this matrix, namely

$$
H_{\partial \Omega}(p)=\sum_{j=1}^{n-1} H_{j j}(p) .
$$

In order to state our result we need to consider the mean of the curvatures in the directions of $T_{p} K$ and $N_{p} K$, namely the numbers $\sum_{i=1}^{k} H_{i i}(p)$ and $\sum_{j=k+1}^{n-1} H_{j j}(p)$.

Theorem 1. Assume that $\partial \Omega$ contains a closed embedded, non-degenerate minimal submanifold $K$ of dimension $k \geq 1$ with $n-k \geq 7$, such that

$$
\bar{H}(p):=2 \sum_{a=1}^{k} H_{a a}(p)+\sum_{j=k+1}^{n-1} H_{j j}(p)>0 \quad \text { for all } \quad p \in K .
$$

Then, for a sequence $d=d_{j} \longrightarrow 0$, Problem (1.11) has a positive solution $u_{d}$ concentrating along $K$ in the sense that expansion (1.12) holds as $d \rightarrow 0$ and besides

$$
d^{2}\left|\nabla u_{d}\right|^{2} \rightarrow S_{n-k} \delta_{K} \quad \text { as } d \rightarrow 0
$$

where $\delta_{K}$ stands for the Dirac measure supported on $K$ and $S_{n-k}$ is an explicit positive constant.

Condition (1.13) is new and unexpected. On the other hand, it is worth noticing that (1.13) can be rewritten as

$$
2 H_{\partial \Omega}(p)-\sum_{j=k+1}^{n-1} H_{j j}(p)>0 \text { for all } p \in K
$$


Formally in the case of point concentration, namely $k=0$, this reduces precisely to $H_{\partial \Omega}(p)>0$, that is exactly the condition known to be necessary for point concentration. We suspect that this condition is essential for the phenomenon to take place. On the other hand, while the high codimension assumption $n-k \geq 7$ is important in our proof, we expect that a similar phenomenon holds just provided that $n-k \geq 5$, and with a suitable change in the bubbling scales for $n-k \geq 3$ (the difference of rates is formally due to the fact that $\int_{\mathbb{R}^{n}} w_{\mu}^{2}$ is finite if and only if $n \geq 5$ ).

It will be convenient to rewrite Problem (1.11) in an equivalent form: Let us set $N=$ $n-k$ and $d^{2}=\varepsilon$. We define

$$
u(x)=\varepsilon^{-\frac{N-2}{4}} v\left(\varepsilon^{-1} x\right) .
$$

Then, setting $\Omega_{\varepsilon}:=\varepsilon^{-1} \Omega$, Problem (1.11) becomes

$$
\begin{cases}\Delta v-\varepsilon v+v^{\frac{N+2}{N-2}}=0 & \text { in } \Omega_{\varepsilon}, \\ \frac{\partial v}{\partial \nu}=0 & \text { on } \partial \Omega_{\varepsilon} .\end{cases}
$$

The proof of the theorem has as a main ingredient the construction of an approximate solution with arbitrary degree of accuracy in powers of $\varepsilon$, in a neighborhood of the manifold $K_{\varepsilon}=\varepsilon^{-1} K$. Later we built the desired solution by linearizing the equation (1.14) around this approximation. The associated linear operator turns out to be invertible with inverse controlled in a suitable norm by certain large negative power of $\varepsilon$, provided that $\varepsilon$ remains away from certain critical values where resonance occurs. The interplay of the size of the error and that of the inverse of the linearization then makes it possible a fixed point scheme.

The accurate approximate solution to (1.14) is built by using an iterative scheme of Picard's type which we describe in general next.

Observe that the desired asymptotic behavior (1.12) translates in terms of $v$ as

$$
v(x) \approx \mu_{0}(\varepsilon z)^{-\frac{N-2}{2}} w_{0}\left(\mu_{0}^{-1}(\varepsilon z)|\zeta|\right), \quad x=z+\zeta, \quad z \in K_{\varepsilon}, \quad|\zeta|=\operatorname{dist}\left(x, K_{\varepsilon}\right),
$$

where

$$
\mu_{0}(y)=a_{N} \bar{H}(y)^{\frac{1}{N-2}} \quad y=\varepsilon z \in K .
$$

Here and in what follows $w_{0}$ designates the standard bubble,

$$
w_{0}(\xi)=w_{0}(|\xi|)=\alpha_{N}\left(\frac{1}{1+|\xi|^{2}}\right)^{\frac{N-2}{2}}, \quad \alpha_{N}=(N(N-2))^{\frac{N-2}{4}} .
$$

We introduce the so-called Fermi coordinates on a neighborhood of $K_{\varepsilon}:=\varepsilon^{-1} K$, as a suitable tool to describe the approximation (1.15). They are defined as follows (we refer 
to Subsection 2.2 for further details): we parameterize a neighborhood of $K_{\varepsilon}$ using the exponential map in $\partial \Omega_{\varepsilon}$

$$
\begin{aligned}
&\left(z, \bar{X}, X_{N}\right) \in K_{\varepsilon} \times \mathbb{R}^{N-1} \times \mathbb{R}_{+} \mapsto \Upsilon\left(z, \bar{X}, X_{N}\right):= \\
& \exp _{z}^{\partial \Omega_{\varepsilon}}\left(\sum_{i=1}^{N-1} X_{i} E_{i}\right)-X_{N} \nu\left(\exp _{z}^{\partial \Omega_{\varepsilon}}\left(\sum_{i=1}^{N-1} X_{i} E_{i}\right)\right)
\end{aligned}
$$

Here the vector fields $E_{i}(z)$ represent an orthonormal basis of $N_{z} K_{\varepsilon}$. Thus, (1.15) corresponds to the statement that after expressing $v$ in these coordinates we get

$$
v\left(z, \bar{X}, X_{N}\right) \approx \mu_{0}(\varepsilon z)^{-\frac{N-2}{2}} w_{0}\left(\mu_{0}^{-1}(\varepsilon z)\left(\bar{X}, X_{N}\right)\right)
$$

where suitable corrections need to be introduced if we want further accuracy on the induced error: we consider a positive smooth function $\mu_{\varepsilon}=\mu_{\varepsilon}(p)$ defined on $K$, a smooth function $\Phi_{\varepsilon}: K \longrightarrow \mathbb{R}^{N-1}$, and the change of variables (with some abuse of notation)

$$
v\left(z, \bar{X}, X_{N}\right)=\mu_{\varepsilon}^{-\frac{N-2}{2}}(\varepsilon z) W\left(\varepsilon^{-1} y, \mu_{\varepsilon}^{-1}(\varepsilon z)\left(\bar{X}-\Phi_{\varepsilon}(\varepsilon z)\right), \mu_{\varepsilon}^{-1}(\varepsilon z) X_{N}\right)
$$

with the new $W$ being a function

$$
W(z, \xi), \quad z=\frac{y}{\varepsilon}, \quad \bar{\xi}=\frac{\bar{X}-\Phi_{\varepsilon}}{\mu_{\varepsilon}}, \quad \xi_{N}=\frac{X_{N}}{\mu_{\varepsilon}} .
$$

We will formally expand $W(z, \xi)$ in powers of $\varepsilon$ starting with $w_{0}(\xi)$, with the functions $\Phi_{\varepsilon}(y)$ and $\mu_{\varepsilon}(y)$ correspondingly expanded.

Substituting into the equation, we will arrive formally to linear equations satisfied by the successive remainders of $w_{0}(\xi)$ (as functions of $\xi$ ). These linear equations involve the basic linearized operator $\mathcal{L}:=-\Delta-p w_{0}^{p-1}$.

The bounded solvability of the linear equations at each step of the iteration, is guaranteed by imposing orthogonality conditions of their right-hand sides, with respect to $\operatorname{ker}(\mathcal{L})$ in $L^{\infty}\left(\mathbb{R}^{N}\right)$. These orthogonality conditions, amount to choices of the coefficients of the expansions of $\mu_{\varepsilon}$ and $\Phi_{\varepsilon}$ : for the latter, the equations involve the Jacobi operator of $K$ and it is where the nondegeneracy assumption is used. The coefficients for the expansion of $\mu_{\varepsilon}(\varepsilon z)$ come from algebraic relations, in particular an orthogonality condition in the first iteration yields

$$
\mu_{0}(y):=a_{N}\left[2 \sum_{j=1}^{k} H_{j j}(y)+\sum_{i=k+1}^{N+k-1} H_{i i}(y)\right] \quad y \in K
$$

This is exactly where the sign condition (1.13) in the theorem appears. 
The rest of the paper is organized as follows. We first introduce some notations and conventions. Next, we collect some notions in differential geometry, like the Fermi coordinates (geodesic normal coordinates) near a minimal submanifold and we expand the coefficients of the metric near these Fermi coordinates. In Section 3 we expand the LaplaceBeltrami operator. Section 4 will be mainly devoted to the construction of the approximate solution to our problem using the local coordinates around the submanifold $K$ introduced before. In Section 5 we define globally the approximation and we write the solution to our problem as the sum of the global approximation plus a remaining term. Thus we express our original problem as a non linear problem in the remaining term. To solve such problem, we need to understand the invertibility properties of a linear operator. To do so we start expanding a quadratic functional associated to the linear problem. In Section 6 we develop a linear theory to study our problem. Then, we turn to the proof of our main theorem in Section 7. Sections 8 and 9 are Appendices, where we postponed the proof of some technical facts to facilitate the reading of the paper.

\section{Geometric setting}

In this section we first introduce Fermi coordinates near a $k$-dimensional submanifold of $\partial \Omega \subset \mathbb{R}^{n}$ (with $n=N+k$ ) and we expand the coefficients of the metric in these coordinates. Then, we recall some basic notions about minimal and non-degenerate submanifold.

\subsection{Notation and conventions}

Dealing with coordinates, Greek letters like $\alpha, \beta, \ldots$, will denote indices varying between 1 and $n-1$, while capital letters like $A, B, \ldots$ will vary between 1 and $n$; Roman letters like $a$ or $b$ will run from 1 to $k$, while indices like $i, j, \ldots$ will run between 1 and $N-1:=$ $n-k-1$.

$\xi_{1}, \ldots, \xi_{N-1}, \xi_{N}$ will denote coordinates in $\mathbb{R}^{N}=\mathbb{R}^{n-k}$, and they will also be written as $\bar{\xi}=\left(\xi_{1}, \ldots, \xi_{N-1}\right), \xi=\left(\bar{\xi}, \xi_{N}\right)$.

The manifold $K$ will be parameterized with coordinates $y=\left(y_{1}, \ldots, y_{k}\right)$. Its dilation $K_{\varepsilon}:=\frac{1}{\varepsilon} K$ will be parameterized by coordinates $z=\left(z_{1}, \ldots, z_{k}\right)$ related to the $y$ 's simply by $y=\varepsilon z$.

Derivatives with respect to the variables $y, z$ or $\xi$ will be denoted by $\partial_{y}, \partial_{z}, \partial_{\xi}$, and for brevity sometimes we might use the symbols $\partial_{a}, \partial_{\bar{a}}$ and $\partial_{i}$ for $\partial_{y_{a}}, \partial_{z_{a}}$ and $\partial_{\xi_{i}}$ respectively. 
In a local system of coordinates, $\left(\bar{g}_{\alpha \beta}\right)_{\alpha \beta}$ are the components of the metric on $\partial \Omega$ naturally induced by $\mathbb{R}^{n}$. Similarly, $\left(\bar{g}_{A B}\right)_{A B}$ are the entries of the metric on $\Omega$ in a neighborhood of the boundary. $\left(H_{\alpha \beta}\right)_{\alpha \beta}$ will denote the components of the mean curvature operator of $\partial \Omega$ into $\mathbb{R}^{n}$.

\subsection{Fermi coordinates on $\partial \Omega$ near $K$ and expansion of the metric}

Let $K$ be a $k$-dimensional submanifold of $(\partial \Omega, \bar{g})(1 \leq k \leq N-1)$. We choose along $K$ a local orthonormal frame field $\left(\left(E_{a}\right)_{a=1, \cdots k},\left(E_{i}\right)_{i=1, \cdots, N-1}\right)$ which is oriented. At points of $K$, we have the natural splitting

$$
T \partial \Omega=T K \oplus N K
$$

where $T K$ is the tangent space to $K$ and $N K$ represents the normal bundle, which are spanned respectively by $\left(E_{a}\right)_{a}$ and $\left(E_{j}\right)_{j}$.

We denote by $\nabla$ the connection induced by the metric $\bar{g}$ and by $\nabla^{N}$ the corresponding normal connection on the normal bundle. Given $p \in K$, we use some geodesic coordinates $y$ centered at $p$. We also assume that at $p$ the normal vectors $\left(E_{i}\right)_{i}, i=1, \ldots, n$, are transported parallely (with respect to $\nabla^{N}$ ) through geodesics from $p$, so in particular

$$
\bar{g}\left(\nabla_{E_{a}} E_{j}, E_{i}\right)=0 \quad \text { at } p, \quad i, j=1, \ldots, n, a=1, \ldots, k .
$$

In a neighborhood of $p$ in $K$, we consider normal geodesic coordinates

$$
f(y):=\exp _{p}^{K}\left(y_{a} E_{a}\right), \quad y:=\left(y_{1}, \ldots, y_{k}\right),
$$

where $\exp ^{K}$ is the exponential map on $K$ and summation over repeated indices is understood. This yields the coordinate vector fields $X_{a}:=f_{*}\left(\partial_{y_{a}}\right)$. We extend the $E_{i}$ along each $\gamma_{E}(s)$ so that they are parallel with respect to the induced connection on the normal bundle $N K$. This yields an orthonormal frame field $X_{i}$ for $N K$ in a neighborhood of $p$ in $K$ which satisfies

$$
\left.\nabla_{X_{a}} X_{i}\right|_{p} \in T_{p} K
$$

A coordinate system in a neighborhood of $p$ in $\partial \Omega$ is now defined by

$$
F(y, \bar{x}):=\exp _{f(y)}^{\partial \Omega}\left(x_{i} X_{i}\right), \quad(y, \bar{x}):=\left(y_{1}, \ldots, y_{k}, x_{1}, \ldots, x_{N-1}\right),
$$

with corresponding coordinate vector fields

$$
X_{i}:=F_{*}\left(\partial_{x_{i}}\right) \quad \text { and } \quad X_{a}:=F_{*}\left(\partial_{y_{a}}\right)
$$

By our choice of coordinates, on $K$ the metric $\bar{g}$ splits in the following way

$$
\bar{g}(q)=\bar{g}_{a b}(q) d y_{a} \otimes d y_{b}+\bar{g}_{i j}(q) d x_{i} \otimes d x_{j}, \quad q \in K
$$


We denote by $\Gamma_{a}^{b}(\cdot)$ the 1 -forms defined on the normal bundle, $N K$, of $K$ by the formula

$$
\bar{g}_{b c} \Gamma_{a i}^{c}:=\bar{g}_{b c} \Gamma_{a}^{c}\left(X_{i}\right)=\bar{g}\left(\nabla_{X_{a}} X_{b}, X_{i}\right) \text { at } q=f(y)
$$

Notice that

$$
K \text { is minimal } \quad \Longleftrightarrow \quad \sum_{a=1}^{k} \Gamma_{a}^{a}\left(E_{i}\right)=0 \quad \text { for any } i=1, \ldots N-1 .
$$

Define $q=f(y)=F(y, 0) \in K$ and let $\left(\tilde{g}_{a b}(y)\right)$ be the induced metric on $K$.

When we consider the metric coefficients in a neighborhood of $K$, we obtain a deviation from formula (2.3), which is expressed by the next lemma. The proof follows the same ideas as Proposition 2.1 in [32] but we give it here for completeness. See also the book [47]. We will denote by $R_{\alpha \beta \gamma \delta}$ the components of the curvature tensor with lowered indices, which are obtained by means of the usual ones $R_{\beta \gamma \delta}^{\sigma}$ by

$$
R_{\alpha \beta \gamma \delta}=\bar{g}_{\alpha \sigma} R_{\beta \gamma \delta}^{\sigma}
$$

Lemma 2.1. At the point $F(y, \bar{x})$, the following expansions hold, for any $a=1, \ldots, k$ and any $i, j=1, \ldots, N-1$, we have

$$
\begin{aligned}
\bar{g}_{i j} & =\delta_{i j}+\frac{1}{3} R_{i s t j} x_{s} x_{t}+\mathcal{O}\left(|x|^{3}\right) ; \\
\bar{g}_{a j} & =\mathcal{O}\left(|x|^{2}\right) ; \\
\bar{g}_{a b} & =\tilde{g}_{a b}-\left\{\tilde{g}_{a c} \Gamma_{b i}^{c}+\tilde{g}_{b c} \Gamma_{a i}^{c}\right\} x_{i}+\left[R_{s a b l}+\tilde{g}_{c d} \Gamma_{a s}^{c} \Gamma_{b l}^{d}\right] x_{s} x_{l}+\mathcal{O}\left(|x|^{3}\right) .
\end{aligned}
$$

Here $R_{\text {istj }}($ see (2.6)) are computed at the point of $K$ parameterized by $(y, 0)$.

Proof. The Fermi coordinates above are defined such that the metric coefficients

$$
g_{\alpha \beta}=g\left(X_{\alpha}, X_{\beta}\right)
$$

is equal to $\delta_{\alpha \beta}$ at $p=F(0,0)$ and $g_{a b}=\tilde{g}_{a b}(y)$ at the point $q=F(y, 0)$ furthermore, $g\left(X_{a}, X_{i}\right)=0$ in some neighborhood of $q$ in $K$. A Taylor expansion of the metric $\bar{g}_{\alpha \beta}(\bar{x}, y)$ at $q$ is given by

$$
\begin{aligned}
\bar{g}_{\alpha \beta} & =\left.\bar{g}\left(X_{\alpha}, X_{\beta}\right)\right|_{q}+\left.X_{j} \bar{g}\left(X_{\alpha}, X_{\beta}\right)\right|_{q} x_{j}+O\left(|x|^{2}\right) \\
& =\left.\bar{g}\left(X_{\alpha}, X_{\beta}\right)\right|_{q}+\left.\bar{g}\left(\nabla_{X_{j}} X_{\alpha}, X_{\beta}\right)\right|_{q} x_{j}+\left.\bar{g}\left(\nabla_{X_{j}} X_{\beta}, X_{\alpha}\right)\right|_{q} x_{j}+O\left(|x|^{2}\right) .
\end{aligned}
$$

Since $\bar{g}\left(X_{b}, X_{i}\right)=0$ in a neighborhood of $q$ we have

$$
\begin{aligned}
0=X_{b} \bar{g}\left(X_{i}, X_{a}\right) & =\bar{g}\left(\nabla_{X_{b}} X_{i}, X_{a}\right)+\bar{g}\left(X_{i}, \nabla_{X_{b}} X_{a}\right) \\
& =\bar{g}\left(\nabla_{X_{i}} X_{b}, X_{a}\right)+\bar{g}\left(X_{i}, \nabla_{X_{b}} X_{a}\right)
\end{aligned}
$$


This implies in particular that

$$
\bar{g}\left(\nabla_{X_{i}} X_{b}, X_{a}\right)=-\bar{g}\left(X_{i}, \nabla_{X_{b}} X_{a}\right)=-\Gamma_{a i}^{c} \tilde{g}_{c b}
$$

Then at first order expansion we have

$$
\begin{aligned}
\bar{g}_{a b} & =\left.\bar{g}\left(X_{a}, X_{b}\right)\right|_{q}+\left.\bar{g}\left(\nabla_{X_{j}} X_{a}, X_{b}\right)\right|_{q} x_{j}+\left.\bar{g}\left(\nabla_{X_{j}} X_{b}, X_{a}\right)\right|_{q} x_{j}+O\left(|x|^{2}\right) \\
& =\tilde{g}_{a b}-\left(\Gamma_{a i}^{c} \tilde{g}_{c b}+\Gamma_{b i}^{c} \tilde{g}_{c a}\right) x_{i}+O\left(|x|^{2}\right) .
\end{aligned}
$$

Similarly using Formula (2.1) we get

$$
\begin{aligned}
\bar{g}_{a i} & =\left.\bar{g}\left(X_{a}, X_{i}\right)\right|_{q}+\left.\bar{g}\left(\nabla_{X_{j}} X_{a}, X_{i}\right)\right|_{q} x_{j}+\left.\bar{g}\left(\nabla_{X_{j}} X_{i}, X_{a}\right)\right|_{q} x_{j}+O\left(|x|^{2}\right) \\
& =O\left(|x|^{2}\right)
\end{aligned}
$$

On the other hand, since every vector field $X \in N_{q} K$ is tangent to the geodesic $s \longrightarrow$ $\exp _{q}^{\partial \Omega}(s X)$, we have

$$
\left.\nabla_{X_{\ell}+X_{j}}\left(X_{\ell}+X_{j}\right)\right|_{q}=0
$$

Which clearly implies that

$$
\left.\left(\nabla_{X_{\ell}} X_{j}+\nabla_{X_{j}} X_{\ell}\right)\right|_{q}=0
$$

Then the following expansion holds

$$
\begin{aligned}
\bar{g}_{i j} & =\left.\bar{g}\left(X_{i}, X_{j}\right)\right|_{q}+\left.\bar{g}\left(\nabla_{X_{l}} X_{i}, X_{j}\right)\right|_{q} x_{l}+\left.\bar{g}\left(\nabla_{X_{l}} X_{j}, X_{i}\right)\right|_{q} x_{l}+O\left(|x|^{2}\right) \\
& =\delta_{i j}+O\left(|x|^{2}\right) .
\end{aligned}
$$

To compute the terms of order two in the Taylor expansion it suffices to compute $X_{k} X_{k} \bar{g}_{\alpha \beta}$ at $q$ and polarize (i.e. replace $X_{k}$ by $X_{i}+X_{j}$ ). We have

$$
X_{k} X_{k} \bar{g}_{\alpha \beta}=\bar{g}\left(\nabla_{X_{k}}^{2} X_{\alpha}, X_{\beta}\right)+\bar{g}\left(X_{\alpha}, \nabla_{X_{k}}^{2} X_{\beta}\right)+2 \bar{g}\left(\nabla_{X_{k}} X_{\alpha}, \nabla_{X_{k}} X_{\beta}\right)
$$

Now, using the fact every normal vector $X \in N_{q} K$ is tangent to the geodesic $s \longrightarrow$ $\exp _{q}^{\partial \Omega}(s X)$, then

$$
\left.\nabla_{X} X\right|_{q}=\left.\nabla_{X}^{2} X\right|_{q}=0
$$

for every $X \in N_{q} K$. In particular, choosing $X=X_{k}+\varepsilon X_{j}$, we obtain

$$
0=\nabla_{X_{k}+\varepsilon X_{j}} \nabla_{X_{k}+\varepsilon X_{j}}\left(X_{k}+\varepsilon X_{j}\right)_{\mid q}
$$

for every $\varepsilon$, which implies $\nabla_{X_{j}} \nabla_{X_{k}} X_{k \mid p}=-2 \nabla_{X_{k}} \nabla_{X_{k}} X_{j \mid p}$, and hence

$$
\left.3 \nabla_{X_{k}}^{2} X_{j}\right|_{q}=\left.R\left(X_{k}, X_{j}\right) X_{k}\right|_{q}
$$


We then deduce from (2.7) that

$$
\left.X_{k} X_{k} \bar{g}_{i j}\right|_{q}=\left.\frac{2}{3} \bar{g}\left(R\left(X_{k}, X_{i}\right) X_{k}, X_{j}\right)\right|_{q}
$$

On the other hand we have

$$
\nabla_{X_{k}}^{2} X_{\gamma}=\nabla_{X_{k}} \nabla_{X_{\gamma}} X_{k}=\nabla_{X_{\gamma}} \nabla_{X_{k}} X_{k}+R\left(X_{k}, X_{\gamma}\right) X_{k}
$$

Hence

$$
\begin{aligned}
X_{k} X_{k} g_{a b} & =2 \bar{g}\left(R\left(X_{k}, X_{a}\right) X_{k}, X_{b}\right)+2 \bar{g}\left(\nabla_{X_{k}} X_{a}, \nabla_{X_{k}} X_{b}\right) \\
& +\bar{g}\left(\nabla_{X_{a}} \nabla_{X_{k}} X_{k}, X_{b}\right)+\bar{g}\left(X_{a}, \nabla_{X_{b}} \nabla_{X_{k}} X_{k}\right) .
\end{aligned}
$$

Now using the fact that $\nabla_{X} X=0_{\mid q}$ at $q \in K$ for every $X \in N_{q} K$, the definition of $\Gamma_{a k}^{c}$ in (2.4) and the formula

$$
R\left(X_{k}, X_{a}\right) X_{l}=R_{k a l}^{\gamma} X_{\gamma}
$$

we deduce that at the point $q$

$$
\begin{aligned}
\left.X_{k} X_{k} \bar{g}_{a b}\right|_{q} & =2 \bar{g}\left(R\left(X_{k}, X_{a}\right) X_{k}, X_{b}\right)+2 \tilde{g}_{c d} \Gamma_{a k}^{c} \Gamma_{b k}^{d} \\
& =2 R_{k a k}^{c} \bar{g}\left(X_{c}, X_{b}\right)+2 \tilde{g}_{c d} \Gamma_{a k}^{c} \Gamma_{b k}^{d} \\
& =2 R_{k a k}^{c} \tilde{g}_{c b}+2 \tilde{g}_{c d} \Gamma_{a k}^{c} \Gamma_{b k}^{d} \\
& =2 R_{k a b k}+2 \tilde{g}_{c d} \Gamma_{a k}^{c} \Gamma_{b k}^{d} .
\end{aligned}
$$

This proves the Lemma.

Next we introduce a parametrization of a neighborhood in $\Omega$ of $q \in \partial \Omega$ through the map $\Upsilon$ given by

$$
\Upsilon(y, x)=F(y, \bar{x})+x_{N} \nu(y, \bar{x}), \quad x=\left(\bar{x}, x_{N}\right) \in \mathbb{R}^{N-1} \times \mathbb{R},
$$

where $F$ is the parametrization introduced in (2.2) and $\nu(y, \bar{x})$ is the inner unit normal to $\partial \Omega$ at $F(y, \bar{x})$. We have

$$
\frac{\partial \Upsilon}{\partial y_{a}}=\frac{\partial F}{\partial y_{a}}(y, \bar{x})+x_{N} \frac{\partial \nu}{\partial y_{a}}(y, \bar{x}) ; \quad \frac{\partial \Upsilon}{\partial x_{i}}=\frac{\partial F}{\partial x_{i}}(y, \bar{x})+x_{N} \frac{\partial \nu}{\partial x_{i}}(y, \bar{x}) .
$$

Let us define the tensor matrix $H$ to be given by

$$
d \nu_{x}[v]=-H(x)[v]
$$

We thus find

$$
\begin{aligned}
& \frac{\partial \Upsilon}{\partial y_{a}}=\left[I d-x_{N} H(y, \bar{x})\right] \frac{\partial F}{\partial y_{a}}(y, \bar{x}) \\
& \frac{\partial \Upsilon}{\partial x_{i}}=\left[I d-x_{N} H(y, \bar{x})\right] \frac{\partial F}{\partial x_{i}}(y, \bar{x}) .
\end{aligned}
$$


Differentiating $\Upsilon$ with respect to $x_{N}$ we also get

$$
\frac{\partial \Upsilon}{\partial x_{N}}=\nu(y, \bar{x})
$$

Hence, letting $g_{\alpha \beta}$ be the coefficients of the flat metric $g$ of $\mathbb{R}^{N+k}$ in the coordinates $\left(y, \bar{x}, x_{N}\right)$, with easy computations we deduce for $\tilde{y}=(y, \bar{x})$ that

$$
\begin{gathered}
g_{\alpha \beta}\left(\tilde{y}, x_{N}\right)=\bar{g}_{\alpha \beta}(\tilde{y})-x_{N}\left(H_{\alpha \delta} \bar{g}_{\delta \beta}+H_{\beta \delta} \bar{g}_{\delta \alpha}\right)(\tilde{y})+x_{N}^{2} H_{\alpha \delta} H_{\sigma \beta} \bar{g}_{\delta \sigma}(\tilde{y}) ; \\
g_{\alpha N} \equiv 0 ; \quad g_{N N} \equiv 1 .
\end{gathered}
$$

In the above expressions, with $\alpha$ and $\beta$ we denote any index of the form $a=1, \ldots, k$ or $i=1, \ldots, N-1$.

We first provide a Taylor expansion of the coefficients of the metric $g$. From Lemma 2.1 and formula (2.13) we have immediately the following result.

Lemma 2.2. For the (Euclidean) metric $g$ in the above coordinates we have the expansions

$$
\begin{aligned}
& g_{i j}=\delta_{i j}-2 x_{N} H_{i j}+\frac{1}{3} R_{i s t j} x_{s} x_{t}+x_{N}^{2}\left(H^{2}\right)_{i j}+\mathcal{O}\left(|x|^{3}\right), \quad 1 \leq i, j \leq N-1 ; \\
& g_{a j}=-x_{N}\left(H_{a j}+\tilde{g}_{a c} H_{c j}\right)+\mathcal{O}\left(|x|^{2}\right), \quad 1 \leq a \leq k, 1 \leq j \leq N-1 ; \\
& g_{a b}=\tilde{g}_{a b}-\left\{\tilde{g}_{a c} \Gamma_{b i}^{c}+\tilde{g}_{b c} \Gamma_{a i}^{c}\right\} x_{i}-x_{N}\left\{H_{a c} \tilde{g}_{b c}+H_{b c} \tilde{g}_{a c}\right\}+\left[R_{s a b l}+\tilde{g}_{c d} \Gamma_{a s}^{c} \Gamma_{d l}^{b}\right] x_{s} x_{l} \\
& +x_{N}^{2}\left(H^{2}\right)_{a b}+x_{N} x_{k}\left[H_{a c}\left\{\tilde{g}_{b f} \Gamma_{c k}^{f}+\tilde{g}_{c f} \Gamma_{b k}^{f}\right\}+H_{b c}\left\{\tilde{g}_{a f} \Gamma_{c k}^{f}+\tilde{g}_{c f} \Gamma_{a k}^{f}\right\}\right]+\mathcal{O}\left(|x|^{3}\right), \\
& 1 \leq a, b \leq k ; \\
& g_{a N} \equiv 0, \quad a=1, \ldots, k ; \quad g_{i N} \equiv 0, \quad i=1, \ldots, N-1 ;
\end{aligned}
$$

In the above expressions $H_{\alpha \beta}$ denotes the components of the matrix tensor $H$ defined in (2.9), $R_{i s t j}$ are the components of the curvature tensor as defined in (2.6), $\Gamma_{a}^{b}\left(E_{i}\right)$ are defined in (2.4). Here we have set

$$
\left(A^{2}\right)_{\alpha \beta}=A_{\alpha i} A_{i \beta}+\tilde{g}_{c d} A_{\alpha c} A_{\beta d}
$$

Furthermore, we have the validity of the following expansion for the log of the determinant of $g$

$$
\begin{aligned}
\log (\operatorname{det} g) & =\log (\operatorname{det} \tilde{g})-2 x_{N} \operatorname{tr}(H)-2 \Gamma_{b k}^{b} x_{k}+\frac{1}{3} R_{m i i l} x_{m} x_{l} \\
& +\left(\tilde{g}^{a b} R_{m a b l}-\Gamma_{a m}^{c} \Gamma_{c l}^{a}\right) x_{m} x_{l}-x_{N}^{2} \operatorname{tr}\left(H^{2}\right)+\mathcal{O}\left(|x|^{3}\right)
\end{aligned}
$$


Recall first that $K$ minimal implies that $\Gamma_{b k}^{b}=0$. The expansions of the metric in the above lemma follow from Lemma 2.1 and formulas (2.13)-(2.14) while the expansion of the log of the determinant of $g$ follows from the fact that $g=G+M$ with

$$
G=\left(\begin{array}{cc}
\tilde{g} & 0 \\
0 & I d_{\mathbb{R}^{n}}
\end{array}\right) \quad \text { and } \quad M=\mathcal{O}(|x|)
$$

then we have the following expansion

$$
\log (\operatorname{det} g)=\log (\operatorname{det} G)+\operatorname{tr}\left(G^{-1} M\right)-\frac{1}{2} \operatorname{tr}\left(\left(G^{-1} M\right)^{2}\right)+O\left(\|M\|^{3}\right) .
$$

We are now in position to give the expansion of the Laplace-beltrami operator. Recall that

$$
\Delta_{g} u=\frac{1}{\sqrt{\operatorname{det} g}} \partial_{\alpha}\left(\sqrt{\operatorname{det} g} g^{\alpha \beta} \partial_{\beta} u\right)
$$

where summation over repeated indices is understood and where $g^{\alpha \beta}$ denotes the entries of the inverse of the metric $\left(g_{\alpha \beta}\right)$. The above formula can be rewritten as

$$
\Delta_{g} u=g^{\alpha \beta} \partial_{\alpha \beta}^{2} u+\partial_{\alpha}\left(g^{\alpha \beta}\right) \partial_{\beta} u+\frac{1}{2} \partial_{\alpha}(\log (\operatorname{det} g)) g^{\alpha \beta} \partial_{\beta} u .
$$

Using the expansions in Lemma 2.2 we have the validity of the following expansion for the Laplace-Beltrami operator

Lemma 2.3. In the above coordinates the Laplace-Beltrami operator can be expanded as

$$
\begin{aligned}
\Delta_{g} u(x, y) & =\Delta_{K} u+\partial_{i i}^{2} u+\partial_{N N}^{2} u-\operatorname{tr}(H) \partial_{N} u+2 x_{N} H_{i j} \partial_{i j}^{2} u \\
& +x_{N}^{2} Q(H)_{i j} \partial_{i j}^{2} u-x_{N} \operatorname{tr}\left(H^{2}\right) \partial_{N} u+2 x_{N} H_{a b} \Gamma_{a i}^{b} \partial_{i} u \\
& +\left(\frac{2}{3} R_{m l l i}+\tilde{g}^{a b} R_{i a b m}-\Gamma_{a m}^{c} \Gamma_{c i}^{a}\right) x_{m} \partial_{i} u-\frac{1}{3} R_{i s l j} x_{s} x_{l} \partial_{i j}^{2} u \\
& +2 x_{N}\left(H_{a j}+\tilde{g}^{a c} H_{c j}\right) \partial_{a j}^{2} u+\left(O\left(|x|^{2}\right)+O(|x|) \partial_{a} \mathfrak{F}^{a \beta}(y, x)\right) \partial_{\beta} u \\
& +\left\{\tilde{g}^{a c} \Gamma_{b i}^{c}+\tilde{g}^{b c} \Gamma_{a i}^{c}\right\} x_{i} \partial_{a b}^{2} u+x_{N}\left\{H_{a c} \tilde{g}^{b c}+H_{b c} \tilde{g}^{a c}\right\} \partial_{a b}^{2} u \\
& +\mathcal{O}\left(|x|^{3}\right) \partial_{i j}^{2} u+\mathcal{O}\left(|x|^{2}\right) \partial_{a j}^{2} u+\mathcal{O}\left(|x|^{2}\right) \partial_{a b}^{2} u .
\end{aligned}
$$

Here the term $Q(H)$ is a quadratic term of $H$ given by

$$
Q(H)_{i j}=3 x_{N}^{2} H_{i k} H_{k j}+x_{N}^{2}\left(2 H_{i a} H_{a j}+\tilde{g}^{a b} H_{i a} H_{b j}\right),
$$

while the term $\mathfrak{F}^{a \beta}(\beta=b$ or $\beta=j)$ is given by the formulas

$$
\mathcal{O}(|x|) \mathfrak{F}^{a b}(y, x)=\left(g^{a b}-\tilde{g}^{a b}\right)+\frac{1}{2}\left(\log (\operatorname{det} g) g^{a b}-\log (\operatorname{det} \tilde{g}) \tilde{g}^{a b}\right)
$$


and

$$
\mathcal{O}(|x|) \mathfrak{F}^{a j}(y, x)=g^{a j}+\frac{1}{2} \log (\operatorname{det} \tilde{g}) g^{a j} .
$$

Proof. Using the expansion of Lemma 2.2 and the fact that if $g=G+M$ with

$$
G=\left(\begin{array}{cc}
\tilde{g} & 0 \\
0 & I d_{\mathbb{R}^{N}}
\end{array}\right) \quad \text { and } \quad M=\mathcal{O}(|x|)
$$

then

$$
g^{-1}=G^{-1}-G^{-1} M G^{-1}+G^{-1} M G^{-1} M G^{-1}+O\left(\|M\|^{3}\right),
$$

it is easy to check that the following expansions hold true

$$
\begin{aligned}
& g^{i j}=\delta_{i j}+2 x_{N} H_{i j}-\frac{1}{3} R_{i s t j} x_{s} x_{t}+x_{N}^{2} Q(H)_{i j}+\mathcal{O}\left(|x|^{3}\right), \quad 1 \leq i, j \leq N-1 ; \\
& g^{a j}=x_{N}\left(H_{a j}+\tilde{g}^{a c} H_{c j}\right)+\mathcal{O}\left(|x|^{2}\right), 1 \leq a \leq k, 1 \leq j \leq N-1 ; \\
& g^{a b}=\tilde{g}^{a b}+\left\{\tilde{g}^{a c} \Gamma_{b i}^{c}+\tilde{g}^{b c} \Gamma_{a i}^{c}\right\} x_{i}+x_{N}\left\{H_{a c} \tilde{g}^{b c}+H_{b c} \tilde{g}^{a c}\right\}+\mathcal{O}\left(|x|^{2}\right), \\
& \quad 1 \leq a, b \leq k ; \quad g^{i N} \equiv 0, \quad i=1, \ldots, N-1 ; \quad a=1, \ldots, k ; \quad g^{N N} \equiv 1 .
\end{aligned}
$$

The proof of the Lemma follows at once.

\subsection{Non degenerate Minimal submanifold}

Denoting by $C^{\infty}(N K)$ the space of smooth normal vector fields on $K$. Then, for $\Phi \in$ $C^{\infty}(N K)$, we define the one-parameter family of submanifolds $t \mapsto K_{t, \Phi}$ by

$$
K_{t, \Phi}:=\left\{\exp _{y}^{\partial \Omega}(t \Phi(y)): y \in K\right\}
$$

The first variation formula of the volume is the equation

$$
\left.\frac{d}{d t}\right|_{t=0} \operatorname{Vol}\left(K_{t, \Phi}\right)=\int_{K}\langle\Phi, \mathbf{h}\rangle_{N} d V_{K}
$$

where $\mathbf{h}$ stands for the mean curvature (vector) of $K$ in $\partial \Omega,\langle\cdot, \cdot\rangle_{N}$ denotes the restriction the metric $\bar{g}$ to $N K$, and $d V_{K}$ the volume element of $K$.

A submanifold $K$ is said to be minimal if it is a critical point for the volume functional, namely if

$$
\left.\frac{d}{d t}\right|_{t=0} \operatorname{Vol}\left(K_{t, \Phi}\right)=0 \quad \text { for any } \Phi \in C^{\infty}(N K)
$$


or, equivalently by (2.17), if the mean curvature $\mathbf{h}$ is identically zero on $K$. It is possible to prove that condition (2.18) is equivalent to (2.5).

The Jacobi operator $\mathfrak{J}$ appears in the expression of the second variation of the volume functional for a minimal submanifold $K$

$$
\left.\frac{d^{2}}{d t^{2}}\right|_{t=0} \operatorname{Vol}\left(K_{t, \Phi}\right)=-\int_{K}\langle\mathfrak{J} \Phi, \Phi\rangle_{N} d V_{K} ; \quad \Phi \in C^{\infty}(N K),
$$

and it is given by

$$
\mathfrak{J} \Phi:=-\Delta_{K}^{N} \Phi+\mathfrak{R}^{N} \Phi-\mathfrak{B}^{N} \Phi,
$$

where $\mathfrak{R}^{N}, \mathfrak{B}^{N}: N K \rightarrow N K$ are defined as

$$
\mathfrak{R}^{N} \Phi=\left(R\left(E_{a}, \Phi\right) E_{a}\right)^{N} ; \quad \bar{g}\left(\mathfrak{B}^{N} \Phi, n_{K}\right):=\Gamma_{b}^{a}(\Phi) \Gamma_{a}^{b}\left(n_{K}\right),
$$

for any unit normal vector $n_{K}$ to $K$. The Jacobi operator defined in (2.20) expressed in Fermi coordinates take the expression

$$
(\mathfrak{J} \Phi)^{l}=-\Delta_{K} \Phi^{l}+\left(\tilde{g}^{a b} R_{\text {mabl }}-\Gamma_{a}^{c}\left(E_{m}\right) \Gamma_{c}^{a}\left(E_{l}\right)\right) \Phi^{m}, \quad l=1, \ldots, N-1
$$

where $R_{\text {maal }}$ and $\Gamma_{a}^{c}\left(E_{m}\right)$ are smooth functions on $K$ and they are defined respectively in (2.6) and (2.4). A submanifold $K$ is said to be non-degenerate if the Jacobi operator $\mathfrak{J}$ is invertible, or equivalently if the equation $\mathfrak{J} \Phi=0$ has only the trivial solution among the sections in $N K$.

\section{Expressing the equation in coordinates}

We recall from (1.14) that we want to find a solution to the problem

$$
\begin{cases}\Delta v-\varepsilon v+v_{+}^{\frac{N+2}{N-2}}=0 & \text { in } \Omega_{\varepsilon}, \\ \frac{\partial v}{\partial \nu}=0 & \text { on } \partial \Omega_{\varepsilon} .\end{cases}
$$

The first element to construct an approximate solution to our problem is the standard bubble

$$
w_{0}(\xi)=\frac{\alpha_{N}}{\left(1+|\xi|^{2}\right)^{\frac{N-2}{2}}}, \quad \alpha_{N}=(N(N-2))^{\frac{N-2}{4}} \quad \text { for all } \quad \xi \in \mathbb{R}^{N}
$$

solution to

$$
\Delta w+w^{\frac{N+2}{N-2}}=0 \quad \text { in } \quad \mathbb{R}_{+}^{N}, \quad \frac{\partial w}{\partial \xi_{N}}=0 \quad \text { in } \quad \partial \mathbb{R}_{+}^{N} .
$$

It is well known that all positive and bounded solutions to (3.3) are given by the family of functions

$$
\mu^{-\frac{N-2}{2}} w_{0}\left(\frac{x-P}{\mu}\right)
$$


for any $\mu>0$ and any point $P=\left(P_{1}, \ldots, P_{N-1}, 0\right) \in \partial \mathbb{R}_{+}^{N}$. The solution we are building will have at main order the shape of a copy of $w_{0}$, centered and translated along the $k$ dimensional manifold $K$ inside $\partial \Omega$. In the original variables in $\Omega$, this approximation will be scaled by a small factor, so that it will turn out to be very much concentrated around the manifold $K$.

To describe this approximation, it will be useful to introduce the following change of variables. Let $(y, x) \in \mathbb{R}^{k+N}$ be the local coordinates along $K$ introduced in (2.8). Let $z=\frac{y}{\varepsilon} \in K_{\varepsilon}$ and $X=\frac{x}{\varepsilon} \in \mathbb{R}^{N}$. A parametrization of a neighborhood (in $\Omega_{\varepsilon}$ ) of $\frac{q}{\varepsilon} \in K_{\varepsilon} \subset \partial \Omega_{\varepsilon}$ close to $K_{\varepsilon}$ is given by the map $\Upsilon_{\varepsilon}$ defined by

$$
\Upsilon_{\varepsilon}\left(z, \bar{X}, X_{N}\right)=\frac{1}{\varepsilon} \Upsilon(\varepsilon z, \varepsilon X), \quad X=\left(\bar{X}, X_{N}\right) \in \mathbb{R}^{N-1} \times \mathbb{R}^{+}
$$

where $\Upsilon$ is the parametrization given in (2.8).

Given a positive smooth function $\mu_{\varepsilon}=\mu_{\varepsilon}(y)$ defined on $K$ and a smooth function $\Phi_{\varepsilon}: K \longrightarrow \mathbb{R}^{N-1}$ defined by $\Phi_{\varepsilon}(y)=\left(\Phi_{\varepsilon}^{1}(y), \ldots, \Phi_{\varepsilon}^{N-1}(y)\right), y \in K$, we consider the change of variables

$$
v\left(z, \bar{X}, X_{N}\right)=\mu_{\varepsilon}^{-\frac{N-2}{2}}(\varepsilon z) W\left(z, \mu_{\varepsilon}^{-1}(\varepsilon z)\left(\bar{X}-\Phi_{\varepsilon}(\varepsilon z)\right), \mu_{\varepsilon}^{-1}(\varepsilon z) X_{N}\right),
$$

with the new $W$ being a function

$$
W=W(z, \xi), \quad z=\frac{y}{\varepsilon}, \quad \bar{\xi}=\frac{\bar{X}-\Phi_{\varepsilon}}{\mu_{\varepsilon}}, \quad \xi_{N}=\frac{X_{N}}{\mu_{\varepsilon}} .
$$

To emphasize the dependence of the above change of variables on $\mu_{\varepsilon}$ and $\Phi_{\varepsilon}$, we will use the notation

$$
v=\mathcal{T}_{\mu_{\varepsilon}, \Phi_{\varepsilon}}(W) \Longleftrightarrow v \quad \text { and } \quad W \quad \text { satisfy (3.5). }
$$

We assume now that the functions $\mu_{\varepsilon}$ and $\Phi_{\varepsilon}$ are uniformly bounded, as $\varepsilon \rightarrow 0$, on $K$. Since the original variables $(y, x) \in \mathbb{R}^{k+N}$ are local coordinates along $K$, we let the variables $(z, \xi)$ vary in the set $\mathcal{D}$ defined by

$$
\mathcal{D}=\left\{\left(z, \bar{\xi}, \xi_{N}\right): \varepsilon z \in K, \quad|\bar{\xi}|<\frac{\delta}{\varepsilon}, \quad 0<\xi_{N}<\frac{\delta}{\varepsilon}\right\}
$$

for some fixed positive number $\delta$. We will also use the notation $\mathcal{D}=K_{\varepsilon} \times \hat{\mathcal{D}}$, where $K_{\varepsilon}=\frac{K}{\varepsilon}$ and

$$
\hat{\mathcal{D}}=\left\{\left(\bar{\xi}, \xi_{N}\right):|\bar{\xi}|<\frac{\delta}{\varepsilon}, \quad 0<\xi_{N}<\frac{\delta}{\varepsilon}\right\} .
$$

Having the expansion of the metric coefficients obtained in Section 2, we easily get the expansion of the metric in the expanded variables: letting $g_{\alpha, \beta}^{\varepsilon}$ be the coefficients of the metric $g^{\varepsilon}$, we have

$$
g_{\alpha, \beta}^{\varepsilon}(z, x)=g_{\alpha, \beta}(\varepsilon z, \varepsilon x)
$$

where $g_{\alpha, \beta}$ are given in Lemma 2.2. With an easy computation we deduce the following 
Lemma 3.1. For the (Euclidean) metric $g^{\varepsilon}$ in the above coordinates $(z, X)$ we have the expansions

$$
\begin{aligned}
& g_{i j}^{\varepsilon}= \delta_{i j}-2 \varepsilon X_{N} H_{i j}+\frac{\varepsilon^{2}}{3} R_{i s t j} X_{s} X_{t}+\varepsilon^{2} X_{N}^{2}\left(H^{2}\right)_{i j}+\mathcal{O}\left(\varepsilon^{3}\left(|X|^{3}\right),\right. \\
& 1 \leq i, j \leq N-1 ; \\
& g_{a j}^{\varepsilon}=-\varepsilon X_{N}\left(H_{a j}+\tilde{g}_{a c}^{\varepsilon} H_{c j}\right)+\mathcal{O}\left(\varepsilon^{2}|X|^{2}\right) \\
& 1 \leq a \leq k, 1 \leq j \leq N-1 ; \\
& g_{a b}^{\varepsilon}=\tilde{g}_{a b}^{\varepsilon}-\varepsilon\left\{\tilde{g}_{a c}^{\varepsilon} \Gamma_{b i}^{c}+\tilde{g}_{b c}^{\varepsilon} \Gamma_{a i}^{c}\right\} X_{i}-\varepsilon X_{N}\left\{H_{a c} \tilde{g}_{b c}^{\varepsilon}+H_{b c} \tilde{g}_{a c}^{\varepsilon}\right\} \\
& \quad+\varepsilon^{2}\left[R_{s a b l}+\tilde{g}_{c d}^{\varepsilon} \Gamma_{a s}^{c} \Gamma_{d l}^{b}\right] X_{s} X_{l}+\varepsilon^{2} X_{N}^{2}\left(H^{2}\right)_{a b} \\
& \quad+\varepsilon^{2} X_{N} X_{k}\left[H_{a c}\left\{\tilde{g}_{b f}^{\varepsilon} \Gamma_{c k}^{f}+\tilde{g}_{c f}^{\varepsilon} \Gamma_{b k}^{f}\right\}+H_{b c}\left\{\tilde{g}_{a f}^{\varepsilon} \Gamma_{c k}^{f}+\tilde{g}_{c f}^{\varepsilon} \Gamma_{a k}^{f}\right\}\right]+\mathcal{O}\left(\varepsilon^{3}|X|^{3}\right), \\
& 1 \leq a, b \leq k ; \quad i \quad g_{i N}^{\varepsilon} \equiv 0, \quad i=1, \ldots, N-1 ; \quad g_{N N}^{\varepsilon} \equiv 1 . \\
& g_{a N}^{\varepsilon} \equiv 0, \quad a=1, \ldots, k ; \quad
\end{aligned}
$$

In the above expressions $H_{\alpha \beta}$ denotes the components of the matrix tensor $H$ defined in (2.9), $R_{\text {istj }}$ are the components of the curvature tensor as defined in (2.6), $\Gamma_{a i}^{b}$ are defined in (2.4) and $\tilde{g}_{a b}^{\varepsilon}(z)=\tilde{g}_{a b}(\varepsilon z)$.

Lemma 3.2. We have the validity of the following expansions for the square root of the determinant of $g^{\varepsilon}$ and the log of determinant of $g^{\varepsilon}$

$$
\begin{aligned}
\sqrt{\operatorname{det} g^{\varepsilon}} & =\sqrt{\operatorname{det} \tilde{g}^{\varepsilon}}\left\{1-\varepsilon X_{N} \operatorname{tr}(H)+\frac{\varepsilon^{2}}{6} R_{m i i l} X_{m} X_{l}+\frac{\varepsilon^{2}}{2}\left(\left(\tilde{g}^{\varepsilon}\right)^{a b} R_{m a b l}-\Gamma_{a m}^{c} \Gamma_{c l}^{a}\right) X_{m} X_{l}\right. \\
& \left.+\frac{\varepsilon^{2}}{2} X_{N}^{2} \operatorname{tr}(H)^{2}-\varepsilon^{2} X_{N}^{2} \operatorname{tr}\left(H^{2}\right)\right\}+\varepsilon^{3} \mathcal{O}\left(|X|^{3}\right)
\end{aligned}
$$

and

$$
\begin{aligned}
\log \left(\operatorname{det} g^{\varepsilon}\right) & =\log \left(\operatorname{det} \tilde{g}^{\varepsilon}\right)-2 \varepsilon X_{N} \operatorname{tr}(H)+\frac{\varepsilon^{2}}{3} R_{m i i l} X_{m} X_{l} \\
& +\varepsilon^{2}\left(\left(\tilde{g}^{\varepsilon}\right)^{a b} R_{m a b l}-\Gamma_{a m}^{c} \Gamma_{c l}^{a}\right) X_{m} X_{l}-\varepsilon^{2} X_{N}^{2} \operatorname{tr}\left(H^{2}\right)+\mathcal{O}\left(\varepsilon^{3}|X|^{3}\right)
\end{aligned}
$$

We are now in a position to expand the Laplace Beltrami operator in the new variables $(z, \xi)$ in terms of the parameter $\varepsilon$, of the functions $\mu_{\varepsilon}(y)$ and $\Phi_{\varepsilon}(y)$. This is the content of next Lemma, whose proof we postpone to Section 8. 
Lemma 3.3. Given the change of variables defined in (3.5), the following expansion for the Laplace Beltrami operator holds true

$$
\mu_{\varepsilon}^{\frac{N+2}{2}} \Delta v=\mathcal{A}_{\mu_{\varepsilon}, \Phi_{\varepsilon}}(W):=\mu_{\varepsilon}^{2} \Delta_{K_{\varepsilon}} W+\Delta_{\xi} W+\sum_{\ell=0}^{5} \mathcal{A}_{\ell} W+B(W) .
$$

Above, the expression $\mathcal{A}_{k}$ denotes the following differential operators

$$
\begin{aligned}
\mathcal{A}_{0} W & =\varepsilon^{2} \mu_{\varepsilon} D_{\bar{\xi}} W\left[\Delta_{K} \Phi_{\varepsilon}\right]-\varepsilon^{2} \mu_{\varepsilon} \Delta_{K} \mu_{\varepsilon}\left(\gamma W+D_{\xi} W[\xi]\right) \\
& +\varepsilon^{2}\left|\nabla_{K} \mu_{\varepsilon}\right|^{2}\left[D_{\xi \xi} W[\xi]^{2}+2(1+\gamma) D_{\xi} W[\xi]+\gamma(1+\gamma) W\right] \\
& +\varepsilon^{2} \nabla_{K} \mu_{\varepsilon} \cdot\left\{2 D_{\bar{\xi} \bar{\xi}} W[\bar{\xi}]+N D_{\bar{\xi}} W\right\}\left[\nabla_{K} \Phi_{\varepsilon}\right]+\varepsilon^{2} D_{\bar{\xi} \bar{\xi}} W\left[\nabla_{K} \Phi_{\varepsilon}\right]^{2} \\
& -2 \varepsilon \mu_{\varepsilon} \tilde{g}^{a b}\left[D_{\xi}\left(\partial_{\bar{a}} W\right)\left[\partial_{b} \mu_{\varepsilon} \xi\right]+D_{\bar{\xi}}\left(\partial_{\bar{a}} W\right)\left[\partial_{b} \Phi_{\varepsilon}\right]+\gamma \partial_{a} \mu_{\varepsilon} \partial_{\bar{b}} W\right]
\end{aligned}
$$

where we have set $\gamma=\frac{N-2}{2}$,

$$
\begin{aligned}
\mathcal{A}_{1} W & =\sum_{i, j}\left[2 \mu_{\varepsilon} \varepsilon H_{i j} \xi_{N}-\frac{\varepsilon^{2}}{3} \sum_{m, l} R_{m i j l}\left(\mu_{\varepsilon} \xi_{m}+\Phi_{\varepsilon}^{m}\right)\left(\mu_{\varepsilon} \xi_{l}+\Phi_{\varepsilon}^{l}\right)\right. \\
& \left.+\mu_{\varepsilon}^{2} \varepsilon^{2} \xi_{N}^{2} Q(H)_{i j}+\mu_{\varepsilon} \varepsilon^{2} \xi_{N} \sum_{l} \mathfrak{D}_{N l}^{i j}\left(\mu_{\varepsilon} \xi_{l}+\Phi_{\varepsilon}^{l}\right)\right] \partial_{i j}^{2} W
\end{aligned}
$$

where the functions $\mathfrak{D}_{N k}^{i j}$ are smooth functions of the variable $z=\frac{y}{\varepsilon}$ and uniformly bounded. Furthermore,

$$
\mathcal{A}_{2} W=\varepsilon^{2} \mu_{\varepsilon} \sum_{j}\left[\sum_{s} \frac{2}{3} R_{m s s j}+\sum_{m, a, b}\left(\tilde{g}_{\varepsilon}^{a b} R_{m a b j}-\Gamma_{a m}^{b} \Gamma_{b j}^{a}\right)\right]\left(\mu_{\varepsilon} \xi_{m}+\Phi_{\varepsilon}^{m}\right) \partial_{j} W
$$

and

$$
\left.\mathcal{A}_{3} W=\left[-\varepsilon \operatorname{tr}(H)-2 \mu_{\varepsilon} \varepsilon^{2} \operatorname{tr}\left(H^{2}\right) \xi_{N}-2 \varepsilon^{2} \sum_{i, a, b}\left(\mu_{\varepsilon} \xi_{i}+\Phi_{\varepsilon}^{i}\right) H_{a b} \Gamma_{b i}^{a}\right)\right] \mu_{\varepsilon} \partial_{N} W .
$$

Moreover

$\mathcal{A}_{4} W=4 \varepsilon \mu_{\varepsilon} \xi_{N} \sum_{a, j} H_{a j}\left(-\varepsilon D_{y}\left(\partial_{j} W\right)\left[\partial_{a} \Phi_{\varepsilon}\right]+\mu_{\varepsilon} \partial_{\bar{a} j}^{2} W-\varepsilon \partial_{a} \mu_{\varepsilon}\left(\gamma \partial_{j} W+D_{\xi}\left(\partial_{j} W\right)[\xi]\right)\right)$

and

$$
\begin{aligned}
\mathcal{A}_{5} W= & \left(\sum_{a, j} \mathfrak{D}_{j}^{a} \varepsilon^{2}\left[\mu_{\varepsilon} \xi_{j}+\Phi_{\varepsilon}^{j}\right]+\varepsilon^{2} \mu_{\varepsilon} \mathfrak{D}_{N}^{a} \xi_{N}\right) \times \\
& \left\{\mu_{\varepsilon}\left[-\varepsilon D_{\bar{\xi}} W\left[\partial_{a} \Phi_{\varepsilon}\right]+\mu_{\varepsilon} \partial_{\bar{a}} W-\varepsilon \partial_{a} \mu_{\varepsilon}\left(\gamma W+D_{\xi} W[\xi]\right)\right]\right\}
\end{aligned}
$$

where $\mathfrak{D}_{j}^{a}$ and $\mathfrak{D}_{N}^{a}$ are smooth functions of $z=\frac{y}{\varepsilon}$. Finally, the operator $B(v)$ is defined below, see (8.1).

We recall that the symbols $\partial_{a}, \partial_{\bar{a}}$ and $\partial_{i}$ denote the derivatives with respect to $\partial_{y_{a}}, \partial_{z_{a}}$ and $\partial_{\xi_{i}}$ respectively. 
After performing the change of variables in (3.5), the original equation in $v$ reduces locally close to $K_{\varepsilon}=\frac{K}{\varepsilon}$ to the following equation in $W$

$$
-\mathcal{A}_{\mu_{\varepsilon}, \Phi_{\varepsilon}} W+\varepsilon \mu_{\varepsilon}^{2} W-W^{p}=0
$$

where $\mathcal{A}_{\mu_{\varepsilon}, \Phi_{\varepsilon}}$ is defined in (3.10) and $p=\frac{N+2}{N-2}$. We denote by $\mathcal{S}_{\varepsilon}$ the operator given by (3.17), namely

$$
\mathcal{S}_{\varepsilon}(v):=-\mathcal{A}_{\mu_{\varepsilon}, \Phi_{\varepsilon}} v+\varepsilon \mu_{\varepsilon}^{2} v-v^{p} .
$$

In order to study equation (3.17) in the set $(z, \xi)$, with $z \in K_{\varepsilon},|\bar{\xi}| \leq \frac{\delta}{\varepsilon}$ and $0<\xi_{N} \leq$ $\frac{\delta}{\varepsilon}$, we will first construct an approximate solution to (3.17) in the whole space $K_{\varepsilon} \times$ $\mathbb{R}^{N-1} \times[0, \infty)$ (see Section 4 ). Then, by using proper cut off functions, we will build a first approximation to (3.17) in the original region $z \in K_{\varepsilon},|\bar{\xi}| \leq \frac{\delta}{\varepsilon}$ and $0<\xi_{N} \leq \frac{\delta}{\varepsilon}$.

The basic tool for this construction is a linear theory we describe below.

Let us recall the well known fact that, due to the invariance under translations and dilations of equation (3.3), and since $w_{0}$ is a non-degenerate solution for (3.3), we have that the functions

$$
Z_{j}(\xi)=\frac{\partial w_{0}}{\partial \xi_{j}}, \quad j=1, \ldots, N-1 \quad \text { and } \quad Z_{0}(\xi)=\xi \cdot \nabla w_{0}(\xi)+\frac{N-2}{2} w_{0}(\xi)
$$

are the only bounded solutions to the linearized equation around $w_{0}$ of problem (3.3)

$$
-\Delta \phi-p w_{0}^{p-1} \phi=0 \quad \text { in } \quad \mathbb{R}^{N-1} \times \mathbb{R}^{+}, \quad \frac{\partial \phi}{\partial \xi_{N}}=0 \quad \text { on } \xi_{N}=0 .
$$

Let us now consider a smooth function $a: K \rightarrow \mathbb{R}$ with $a(y) \geq \lambda>0$ for all $y \in K$ and a function $g: K \times \mathbb{R}^{N-1} \times \mathbb{R}^{+} \rightarrow \mathbb{R}$ that depends smoothly on the variable $y \in K$. Recall that a variable $z \in K_{\varepsilon}$ has the form $\varepsilon z=y \in K$.

We want to find a linear theory for the following linear problem

$$
\begin{cases}-\Delta_{\mathbb{R}^{N}} \phi-p w_{0}^{p-1} \phi+\varepsilon a(\varepsilon z) \phi=g & \text { in } \mathbb{R}_{+}^{N} \\ \frac{\partial \phi}{\partial \xi_{N}}=0, & \text { on }\left\{\xi_{N}=0\right\} \\ \int_{\mathbb{R}^{N-1} \times[0, \infty)} \phi(\varepsilon z, \xi) Z_{j}(\xi) d \xi=0 & \text { for all } z \in K_{\varepsilon}, j=0, \ldots N-1 .\end{cases}
$$

To do so we first define the following norms: Let $\delta>0$ be a positive small fixed number and $r$ be a positive number. For a function $w$ defined in $K_{\varepsilon} \times \mathbb{R}^{N-1} \times[0, \infty)$, we define

$$
\|w\|_{\varepsilon, r}:=\sup _{(z, \xi) \in K_{\varepsilon} \times\left\{|\xi| \leq \frac{\delta}{\sqrt{\varepsilon}}\right\}}\left(\left(1+|\xi|^{2}\right)^{\frac{r}{2}}|w(z, \xi)|\right)+\sup _{(z, \xi) \in K_{\varepsilon} \times\left\{|\xi| \geq \frac{\delta}{\sqrt{\varepsilon}}\right\}}\left(\varepsilon^{-\frac{r}{2}}|w(z, \xi)|\right) .
$$


Let $\sigma \in(0,1)$. We further define

$$
\begin{aligned}
\|w\|_{\varepsilon, r, \sigma} & :=\|w\|_{\varepsilon, r}+\sup _{(z, \xi) \in K_{\varepsilon} \times\left\{|\xi| \leq \frac{\delta}{\sqrt{\varepsilon}}\right\}}\left(\left(1+|\xi|^{2}\right)^{\frac{r+\sigma}{2}}[w]_{\sigma, B(\xi, 1)}\right) \\
& +\sup _{(z, \xi) \in K_{\varepsilon} \times\left\{|\xi| \geq \frac{\delta}{\sqrt{\varepsilon}}\right\}}\left(\varepsilon^{-\frac{r+\sigma}{2}}[w]_{\sigma, B(\xi, 1)}\right)
\end{aligned}
$$

where we have set

$$
[w]_{\sigma, B(\xi, 1)}:=\sup _{\xi_{1}, \xi_{2} \in B(\xi, 1)} \frac{\left|w\left(z, \xi_{2}\right)-w\left(z, \xi_{1}\right)\right|}{\left|\xi_{1}-\xi_{2}\right|^{\sigma}} .
$$

We have the validity of the following lemma.

Lemma 3.4. Let $r$ be a number with $2<r<N$ and $\sigma \in(0,1)$. Let $a: K \rightarrow \mathbb{R}$ be $a$ smooth function, such that $a(y) \geq \lambda>0$ for all $y \in K$. Let $g: K \times \mathbb{R}^{N-1} \times[0, \infty) \rightarrow \mathbb{R}$ be a function that depends smoothly on the variable $y \in K$, such that $\|g\|_{\varepsilon, r}<+\infty$ and

$$
\int_{\mathbb{R}^{N-1} \times[0, \infty)} g(\varepsilon z, \xi) Z_{j}(\xi) d \xi=0 \quad \text { for all } \quad z \in K_{\varepsilon}, \quad j=0, \ldots N-1 .
$$

Then there exist a positive constant $C$ such that for all sufficiently small $\varepsilon$ there is a solution $\phi$ to Problem (3.20) such that

$$
\left\|D_{\xi}^{2} \phi\right\|_{\varepsilon, r, \sigma}+\left\|D_{\xi} \phi\right\|_{\varepsilon, r-1, \sigma}+\|\phi\|_{\varepsilon, r-2, \sigma} \leq C\|g\|_{\varepsilon, r, \sigma}
$$

Furthermore, the function $\phi$ depends smoothly on the variable $\varepsilon z$, and the following estimates hold true: for any integer l there exists a positive constant $C_{l}$ such that

$$
\left\|D_{z}^{l} \phi\right\|_{\varepsilon, r-2, \sigma} \leq C_{l}\left(\sum_{k \leq l}\left\|D_{z}^{k} g\right\|_{\varepsilon, r, \sigma}\right) .
$$

Proof. The proof of this lemma will be divided into several steps.

Step 1. We start showing the validity of an a-priori external estimate for a solution to problem (3.20). Assume $\phi$ is a solution to problem (3.20). Given $R>0$, we claim that

$$
|\phi(z, \xi)| \leq C\left(\|\phi\|_{L^{\infty}\left(|\xi|=\delta R \varepsilon^{\left.-\frac{1}{2}\right)}\right.}+\varepsilon^{\frac{r-2}{2}}\|g\|_{\varepsilon, r}\right)
$$

for all $z \in K_{\varepsilon}$ and $|\xi|>R \delta \varepsilon^{-\frac{1}{2}}$.

Let $R>0$ be a fixed number, independent of $\varepsilon$. In the region $|\xi|>R \delta \varepsilon^{-\frac{1}{2}}$ the function $\phi$ solves

$$
-\Delta \phi+\varepsilon b(\varepsilon z, \xi) \phi=g
$$


where

$$
b(\varepsilon z, \xi)=a(\varepsilon z)-\frac{p w_{0}^{p-1}}{\varepsilon}=a(\varepsilon z)+\varepsilon \Theta_{\varepsilon}(\xi),
$$

with $\Theta_{\varepsilon}$ a function uniformly bounded in the considered region, as $\varepsilon \rightarrow 0$. Thus we have that $b$ uniformly positive and bounded as $\varepsilon \rightarrow 0$. Using the maximum principle, we get

$$
\begin{gathered}
\|\phi\|_{L^{\infty}\left(|\xi|>\delta R \varepsilon^{-\frac{1}{2}}\right)} \leq C\left(\varepsilon^{-1}\|g\|_{L^{\infty}\left(|\xi|>\delta R \varepsilon^{-\frac{1}{2}}\right)}+\|\phi\|_{L^{\infty}\left(|\xi|=\delta R \varepsilon^{-\frac{1}{2}}\right)}\right) \\
\leq C\left(\varepsilon^{\frac{r-2}{2}}\|g\|_{\varepsilon, r}+\|\phi\|_{L^{\infty}\left(|\xi|=\delta R \varepsilon^{-\frac{1}{2}}\right)}\right)
\end{gathered}
$$

which gives (3.26).

Step 2. Assume now that $\phi$ is a solution to (3.20). We will now prove that there exists $C>0$ such that

$$
\|\phi\|_{\varepsilon, r-2} \leq C\|g\|_{\varepsilon, r} .
$$

We argue by contradiction: let us assume that there exist sequences $\varepsilon_{n} \rightarrow 0, g_{n}$ with $\left\|g_{n}\right\|_{\varepsilon_{n}, r} \rightarrow 0$ and solutions $\phi_{n}$ to (3.20) with $\left\|\phi_{n}\right\|_{\varepsilon_{n}, r-2}=1$.

Let $z_{n} \in K_{\varepsilon_{n}}$ and $\xi_{n}$ be such that

$$
\left|\phi_{n}\left(\varepsilon_{n} z_{n}, \xi_{n}\right)\right|=\sup \left|\phi_{n}(y, \xi)\right|
$$

We may assume that, up to subsequences, $\left(\varepsilon_{n} z_{n}\right) \rightarrow \bar{y}$ in $K$. On the other hand, from Step 1 , we get that

$$
\sup _{z \in K_{\varepsilon_{n}},|\xi|>\delta R \varepsilon_{n}^{-\frac{1}{2}}} \varepsilon_{n}^{\frac{r-2}{2}}\left|\phi_{n}\left(\varepsilon_{n} z, \xi\right)\right| \leq C R^{-\frac{r-2}{2}},
$$

thus choosing $R$ sufficiently large, but independent of $\varepsilon_{n}$, we have that

$$
\sup _{z \in K_{\varepsilon_{n}},|\xi|>\delta R \varepsilon_{n}^{-\frac{1}{2}}} \varepsilon_{n}^{\frac{r-2}{2}}\left|\phi_{n}(z, \xi)\right|
$$

is arbitrarily small. In particular one gets that $\left|\xi_{n}\right| \leq C \varepsilon_{n}^{-\frac{1}{2}}$ for some positive constant $C$ independent of $\varepsilon_{n}$.

Let us now assume that there exists a positive constant $M$ such that $\left|\xi_{n}\right| \leq M$. In this case, up to subsequences, one gets that $\xi_{n} \rightarrow \xi_{0}$. We then consider the functions $\tilde{\phi}_{n}(z, \xi)=\phi_{n}\left(z, \xi+\xi_{n}\right)$. This is a sequence of uniformly bounded functions, and the sequence $\left(\tilde{\phi}_{n}\right)$ converges uniformly over compact sets of $K \times \mathbb{R}^{N-1} \times \mathbb{R}^{+}$to a function $\tilde{\phi}$ solution to

$$
\begin{cases}-\Delta \tilde{\phi}-p w_{0}^{p-1} \tilde{\phi}=0 & \text { in } \mathbb{R}_{+}^{N} \\ \frac{\partial \tilde{\phi}}{\partial \xi_{N}}=0, & \text { on }\left\{\xi_{N}=0\right\}\end{cases}
$$

Since the orthogonality conditions pass to the limit, we get that furthermore

$$
\int_{\mathbb{R}^{N-1} \times[0, \infty)} \tilde{\phi}(y, \xi) Z_{j}(\xi) d \xi=0 \quad \text { for all } \quad y \in K, \quad \text { for all } \quad j=0, \ldots N-1
$$


These facts imply that $\tilde{\phi} \equiv 0$, that is a contradiction.

Assume now that $\lim _{n \rightarrow \infty}\left|\xi_{n}\right|=\infty$ and define the function $\tilde{\phi}_{n}(z, \xi)=\phi_{n}\left(z,\left|\xi_{n}\right| \xi+\xi_{n}\right)$. Clearly $\tilde{\phi}_{n}$ satisfies the equation

$$
\Delta \tilde{\phi}_{n}+p C_{N} \frac{\left|\xi_{n}\right|^{2}}{\left(1+|| \xi_{n}\left|\xi+\xi_{n}\right|^{2}\right)^{2}} \tilde{\phi}_{n}-\left|\xi_{n}\right|^{2} \varepsilon_{n} a \tilde{\phi}_{n}=\left|\xi_{n}\right|^{2} g\left(z,\left|\xi_{n}\right| \xi+\xi_{n}\right)
$$

Consider first the case in which $\lim _{n \rightarrow \infty} \varepsilon_{n}\left|\xi_{n}\right|^{2}=0$. Under our assumptions, we have that $\tilde{\phi}_{n}$ is uniformly bounded and it converges locally over compact sets to $\tilde{\phi}$ solution to

$$
\Delta \tilde{\phi}=0 \quad \text { in } \quad \mathbb{R}^{N}, \quad|\tilde{\phi}| \leq C|\xi|^{2-r}
$$

Since $2<r<N$, we conclude that $\tilde{\phi} \equiv 0$, which is a contradiction.

Consider now the other possible case, namely that $\lim _{n \rightarrow \infty} \varepsilon_{n}\left|\xi_{n}\right|^{2}=\beta>0$. Then, up to subsequences we get that $\tilde{\phi}_{n}$ converges uniformly over compact sets to $\tilde{\phi}$ solution to

$$
\Delta \tilde{\phi}-\beta a \tilde{\phi}=0 \quad \text { in } \quad \mathbb{R}^{N}, \quad|\tilde{\phi}| \leq C|\xi|^{2-r} .
$$

This implies that $\tilde{\phi} \equiv 0$, which is a contradiction. Taking into account the result of Step 1 , the proof of (3.27) is completed.

Step 3. We shall now show that there exists $C>0$ such that, if $\phi$ is a solution to (3.20), then

$$
\left\|D_{\xi} \phi\right\|_{\varepsilon, r-1}+\|\phi\|_{\varepsilon, r-2} \leq C\|g\|_{\varepsilon, r} .
$$

Let us first assume we are in the region $|\xi|<\delta \varepsilon^{-\frac{1}{2}}$, and $z \in K_{\varepsilon}$. Thus, using estimate (3.27), we have that $\phi$ solves $-\Delta \phi=\hat{g}$ in $|\xi|<\delta \varepsilon^{-\frac{1}{2}}$ where $|\hat{g}| \leq \frac{\|g\|_{\varepsilon, r}}{\left(1+|\xi|^{r}\right)}$.

Let us now fix a point $e \in \mathbb{R}^{N},|e|=1$ and a positive number $R>0$. Perform the change of variables $\tilde{\phi}(z, t)=R^{r-2} \phi(z, R t+3 R e)$, so that

$$
\Delta \tilde{\phi}=\tilde{g} \quad \text { in } \quad|t| \leq 1
$$

where $\tilde{g}(t, z)=R^{r} \hat{g}(z, R t+3 R e)$, so that

$$
\|\tilde{\phi}\|_{L^{\infty}(B(0,2))}+\|\tilde{g}\|_{L^{\infty}(B(0,2))} \leq\|g\|_{\varepsilon, r}
$$

Elliptic estimates give then that $\|D \tilde{\phi}\|_{L^{\infty}(B(0,1))} \leq C\|\tilde{g}\|_{L^{\infty}(B(0,2))}$. This inequality implies then that

$$
\left\|(1+|\xi|)^{r-1} D \phi\right\|_{L^{\infty}\left(|\xi| \leq \delta \varepsilon^{-\frac{1}{2}}\right)} \leq C\left\|(1+|\xi|)^{r} g\right\|_{L^{\infty}\left(|\xi| \leq 2 \delta \varepsilon^{-\frac{1}{2}}\right)}
$$

Assume now that $|\xi|>\delta \varepsilon^{-\frac{1}{2}}$. In this region the function $\phi$ solves

$$
-\Delta \phi=\hat{g}
$$


where $|\hat{g}| \leq C\|g\|_{\varepsilon, r} \varepsilon^{\frac{r}{2}}$, and $|\phi| \leq C\|g\|_{\varepsilon, r} \varepsilon^{\frac{r-2}{2}}$. After scaling out $\varepsilon^{\frac{1}{2}}$, elliptic estimates yield $|D \phi| \leq C \varepsilon^{\frac{r-1}{2}}$. This concludes the proof of (3.28).

Step 4. We shall now show that

$$
\left\|D_{\xi}^{2} \phi\right\|_{\varepsilon, r, \sigma}+\left\|D_{\xi} \phi\right\|_{\varepsilon, r-1, \sigma}+\|\phi\|_{\varepsilon, r-2, \sigma} \leq C\|g\|_{\varepsilon, r, \sigma} .
$$

Let us first assume we are in the region $|\xi|<\delta \varepsilon^{-\frac{1}{2}}$. Thus, we write that $\phi$ solves $-\Delta \phi=\hat{g}$ in $|\xi|<\delta \varepsilon^{-\frac{1}{2}}$ where, thanks to the $C^{1}$-estimate of Step 3, $\|\tilde{g}\|_{\varepsilon, r, \sigma} \leq C\|g\|_{\varepsilon, r, \sigma}$.

Arguing as in the previous step, we fix a point $e \in \mathbb{R}^{N},|e|=1$ and a positive number $R>0$ and let $\tilde{\phi}$ and $\tilde{g}$ be defined as in Step 3. Elliptic estimates give then that $\left\|D^{2} \tilde{\phi}\right\|_{C^{0, \sigma}(B(0,1))} \leq C\|\tilde{g}\|_{C^{0, \sigma}(B(0,2))}$. This inequality gets translated in terms of $\phi$ as the desired Schauder estimate within $|\xi| \leq \delta \varepsilon^{-\frac{1}{2}}$. The Hölder estimate for $D \phi$ follows by interpolation. In the region $|\xi|>\delta \varepsilon^{-\frac{1}{2}}$, we argue exactly as in the proof of Step 3. This concludes the proof of (3.29).

Step 5. Now we shall prove the existence of the solution $\phi$ to problem (3.20). We consider first the following auxiliary problem: find $\bar{\phi}$ and $\alpha: K_{\varepsilon} \rightarrow \mathbb{R}$ solution to

$$
\left\{\begin{array}{lc}
-\Delta \bar{\phi}-p w_{0}^{p-1} \bar{\phi}+\varepsilon a(y) \bar{\phi}=g+\alpha(z) Z(\xi) & \text { in } \mathbb{R}_{+}^{N} \\
\frac{\partial \bar{\phi}}{\partial \xi_{N}}=0, & \text { on }\left\{\xi_{N}=0\right\} \\
\int_{\mathbb{R}^{N-1} \times \mathbb{R}^{+}} \bar{\phi}(z, \xi) Z_{j}(\xi) d \xi=\int_{\mathbb{R}^{N-1} \times \mathbb{R}^{+}} \bar{\phi}(z, \xi) Z(\xi) d \xi=0 & z \in K_{\varepsilon}, \\
(j=0, \ldots N-1), &
\end{array}\right.
$$

where $Z$ is the first eigenfunction, with corresponding first eigenvalue $\lambda_{0}>0$, in $L^{2}\left(\mathbb{R}^{N}\right)$ of the problem

$$
\Delta_{\xi} \bar{\phi}+p w_{0}(\xi)^{p-1} \bar{\phi}=\lambda \bar{\phi} \quad \text { in } \quad \mathbb{R}^{N}
$$

The above problem is variational: for any fixed $z \in K_{\varepsilon}$, solutions to (3.30) correspond to critical points of the energy functional

$$
E(\bar{\phi})=\frac{1}{2} \int_{\mathbb{R}^{N-1} \times \mathbb{R}^{+}}|\nabla \bar{\phi}|^{2}-p w_{0}^{p-1} \bar{\phi}^{2}+\varepsilon a \bar{\phi}^{2}-\int_{\mathbb{R}^{N-1} \times \mathbb{R}^{+}} g \bar{\phi}
$$

for functions $\bar{\phi} \in H^{1}\left(\mathbb{R}^{N-1} \times \mathbb{R}^{+}\right)$, that satisfy

$$
\int_{\mathbb{R}^{N-1} \times \mathbb{R}^{+}} \bar{\phi} Z_{j}=\int_{\mathbb{R}^{N-1} \times \mathbb{R}^{+}} \bar{\phi} Z=0
$$

for all $j=0, \ldots, N-1$. This functional is smooth, uniformly bounded from below, and satisfies the Palais-Smale condition. We thus conclude that $E$ has a minimum, which gives a solution to (3.30).

Observe now that, multiplying the equation in (3.30) against $Z$, integrating in $\mathbb{R}^{N-1} \times$ $\mathbb{R}^{+}$, and using the orthogonality conditions in (3.30), one easily gets that

$$
\alpha(z)=\int_{\mathbb{R}^{N-1} \times \mathbb{R}^{+}} g(z, \xi) Z(\xi) d \xi \quad \text { for all } \quad z \in K_{\varepsilon} .
$$


Given $(\bar{\phi}, \alpha)$ solution to (3.30), we define

$$
\phi=\bar{\phi}+\beta Z \quad \text { with } \quad \beta(z)=\frac{\int g(z, \xi) Z(\xi) d \xi}{\lambda_{0}+\varepsilon a(\varepsilon z)} .
$$

A straightforward computation shows that $\phi$ is a solution to (3.30).

Finally, estimate (3.25) follows by a direct differentiation of equation (3.20) and a use of estimate (3.24). This concludes the proof of Lemma 3.4.

\section{Construction of approximate solutions}

This section will be devoted to build an approximate solution to Problem (3.17) locally close to $K_{\varepsilon}$, using an iterative method that we describe below: Let $I$ be an integer. The expanded variables $(z, \xi)$ will be defined as in (3.6) with

$$
\mu_{\varepsilon}(\varepsilon z)=\mu_{0}+\mu_{1, \varepsilon}+\cdots+\mu_{I, \varepsilon}
$$

where $\mu_{0}, \mu_{1, \varepsilon}, \ldots, \mu_{I, \varepsilon}$ are smooth functions on $K$, with $\mu_{0}$ positive, and

$$
\Phi_{\varepsilon}(\varepsilon z)=\Phi_{1, \varepsilon}+\cdots+\Phi_{I, \varepsilon}
$$

where $\Phi_{1, \varepsilon}, \ldots, \Phi_{I, \varepsilon}$ are smooth functions defined along $K$ with values in $\mathbb{R}^{N-1}$. In the $(z, \xi)$ variables, the shape of the approximate solution will be given by

$$
W_{I+1, \varepsilon}\left(z, \bar{\xi}, \xi_{N}\right)=w_{0}(\xi)+w_{1, \varepsilon}(z, \xi)+\cdots+w_{I+1, \varepsilon}(z, \xi), \quad \xi=\left(\bar{\xi}, \xi_{N}\right)
$$

where $w_{0}$ is given by (3.2) and the functions $w_{j, \varepsilon}$ 's for $j \geq 1$ are to be determined so that the above function $W_{I+1, \varepsilon}$ satisfies formally

$$
-\mathcal{A}_{\mu_{\varepsilon}, \Phi_{\varepsilon}} W_{I+1, \varepsilon}+\varepsilon \mu_{\varepsilon}^{2} W_{I+1, \varepsilon}-W_{I+1, \varepsilon}^{\frac{N+2}{N-2}}=\mathcal{O}\left(\varepsilon^{1+\frac{I}{2}}\right) \quad \text { in } \quad K_{\varepsilon} \times \mathbb{R}^{N-1} \times \mathbb{R}^{+} .
$$

This can be done expanding the equation (3.17) formally in powers of $\varepsilon$ (and in terms of $\mu_{\varepsilon}$ and $\Phi_{\varepsilon}$ ) for $W=W_{I+1, \varepsilon}$ (using basically Lemma 3.3) and analyzing each term separately. For example, looking at the coefficient of $\varepsilon$ in the expansion we will determine $\mu_{0}$ and $w_{1 \varepsilon}$, while looking at the coefficient of $\varepsilon^{1+\frac{j}{2}}$ we will determine $w_{j, \varepsilon}, \mu_{j-1, \varepsilon}$ and $\Phi_{j-1, \varepsilon}$, for $j=2, \ldots, I+1$. In this procedure we use crucially the non degeneracy assumption on $K$ (which implies the invertibility of the Jacobi operator) when considering the projection on some elements of the kernel of the linearization of (1.14) at $w_{0}$, while when projecting on the remaining part of the kernel we have to choose the functions $\mu_{j, \varepsilon}$. This Section is devoted to do this construction. 
Lemma 4.1. For any integer $I \in \mathbb{N}$ there exist smooth functions $\mu_{\varepsilon}: K \rightarrow \mathbb{R}$ and $\Phi_{\varepsilon}: K \rightarrow \mathbb{R}^{N-1}$, such that

$$
\begin{aligned}
& \left\|\mu_{\varepsilon}\right\|_{L^{\infty}(K)}+\left\|\partial_{a} \mu_{\varepsilon}\right\|_{L^{\infty}(K)}+\left\|\partial_{a}^{2} \mu_{\varepsilon}\right\|_{L^{\infty}(K)} \leq C \\
& \left\|\Phi_{\varepsilon}\right\|_{L^{\infty}(K)}+\left\|\partial_{a} \Phi_{\varepsilon}\right\|_{L^{\infty}(K)}+\left\|\partial_{a}^{2} \Phi_{\varepsilon}\right\|_{L^{\infty}(K)} \leq C
\end{aligned}
$$

for some positive constant $C$, independent of $\varepsilon$. In particular, we have

$$
\left\|\mu_{\varepsilon}-\mu_{0}\right\|_{L^{\infty}(K)} \rightarrow 0, \quad\left\|\Phi_{\varepsilon}-\Phi_{0}\right\|_{L^{\infty}(K)} \rightarrow 0
$$

where $\mu_{0}$ is the function defined explicitly as

$$
\mu_{0}(y)=\frac{\int_{\mathbb{R}_{+}^{N}} \xi_{N}\left|\partial_{1} w_{0}\right|^{2}}{\int_{\mathbb{R}_{+}^{N}} w_{0}^{2}}\left[2 H_{a a}(y)+H_{i i}(y)\right]
$$

By assumption (1.13), it turns out that the function $\mu_{0}$ is strictly positive along $K$. Moreover $\Phi_{0}$ is a smooth function along $K$ with values in $\mathbb{R}^{N-1}$. Furthermore there exists a positive function $W_{I+1, \varepsilon}: K_{\varepsilon} \times \mathbb{R}_{+}^{N} \rightarrow \mathbb{R}$ such that

$$
\begin{gathered}
\mathcal{A}_{\mu_{\varepsilon}, \Phi_{\varepsilon}}\left(W_{I+1, \varepsilon}\right)-\varepsilon \mu_{\varepsilon}^{2} W_{I+1, \varepsilon}+W_{I+1, \varepsilon}^{p}=\mathcal{E}_{I+1, \varepsilon} \text { in } K_{\varepsilon} \times \mathbb{R}_{+}^{N} \\
\frac{\partial W_{I+1, \varepsilon}}{\partial \nu}=0 \quad \text { on } \partial \mathbb{R}_{+}^{N}
\end{gathered}
$$

with

$$
\left\|W_{I+1, \varepsilon}-W_{I, \varepsilon}\right\|_{\varepsilon, N-4, \sigma} \leq C \varepsilon^{1+\frac{I}{2}}
$$

and

$$
\left\|\mathcal{E}_{I+1, \varepsilon}\right\|_{\varepsilon, N-2, \sigma} \leq C \varepsilon^{1+\frac{I+1}{2}}
$$

We should emphasize that $\int w_{0}^{2}$ is indeed finite thanks to the fact that $N \geq 5$, and we are actually assuming that $N \geq 7$. The rest of this Section is devoted to do this construction.

- Construction of $w_{1, \varepsilon}$ : Using Lemma 3.3, we formally have

$$
\begin{aligned}
-\mathcal{A}_{\mu_{\varepsilon}, \Phi_{\varepsilon}} W_{1, \varepsilon}+\varepsilon \mu_{\varepsilon}^{2} W_{1, \varepsilon}-W_{1, \varepsilon}^{p} & =-\Delta_{\mathbb{R}^{N}} w_{0}-w_{0}^{p} \\
& +\left(-\Delta_{\mathbb{R}^{N}} w_{1, \varepsilon}-p w_{0}^{p-1} w_{1, \varepsilon}+\varepsilon \mu_{0}^{2} w_{1, \varepsilon}\right) \\
& +\varepsilon \mu_{0}\left[H_{\alpha \alpha} \partial_{\xi_{N}} w_{0}-2 \xi_{N} H_{i j} \partial_{i j}^{2} w_{0}+\mu_{0}(y) w_{0}\right] \\
& +\mathcal{E}_{1, \varepsilon}+Q_{\varepsilon}\left(w_{1, \varepsilon}\right),
\end{aligned}
$$

where $\mathcal{E}_{1, \varepsilon}$ is a sum of functions of the form

$$
\varepsilon \mu_{0}\left(\varepsilon \mu_{0}+\varepsilon \partial_{a} \mu_{0}+\varepsilon \partial_{a}^{2} \mu_{0}\right) a(z) b(\xi)
$$


and $a(\varepsilon z)$ is a smooth function uniformly bounded, together with its derivatives, as $\varepsilon \rightarrow 0$, while the function $b$ is such that

$$
\sup _{\xi}\left(1+|\xi|^{N-2}\right)|b(\xi)|<\infty
$$

The term $Q_{\varepsilon}\left(w_{1, \varepsilon}\right)$ is quadratic in $w_{1, \varepsilon}$, in fact it is explicitly given by

$$
-\left(w_{0}+w_{1, \varepsilon}\right)^{p}+w_{0}^{p}+p w_{0}^{p-1} w_{1, \varepsilon}
$$

Observe now that the term of order 0 (in the power expansion in $\varepsilon$ ) vanishes because of the equation satisfied by $w_{0}$. In order to make the coefficient of $\varepsilon$ vanish, $w_{1, \varepsilon}$ must satisfy the following equation

$$
\begin{cases}-\Delta w_{1, \varepsilon}-p w_{0}^{p-1} w_{1, \varepsilon}+\varepsilon \mu_{0}^{2} w_{1, \varepsilon}=\varepsilon g_{1, \varepsilon}(\varepsilon z, \xi) & \text { in } \mathbb{R}_{+}^{N}, \\ \frac{\partial w_{1, \varepsilon}}{\partial \xi_{N}}=0, & \text { on }\left\{\xi_{N}=0\right\}\end{cases}
$$

where

$$
g_{1, \varepsilon}(\varepsilon z, \xi)=-\mu_{0}(y)\left[H_{\alpha \alpha} \partial_{\xi_{N}} w_{0}-2 \xi_{N} H_{i j} \partial_{i j}^{2} w_{0}+\mu_{0}(y) w_{0}\right] .
$$

Using Lemma 3.4, we see that equation (4.9) is solvable if the right-hand side is $L^{2}$ orthogonal to the functions $Z_{j}$, for $j=0, \ldots, N-1$. These conditions, for $j=1, \ldots, N-$ 1 are clearly satisfied since both $\partial_{N} w_{0}$ and $\partial_{i j}^{2} w_{0}$ are even in $\bar{\xi}$, while the $Z_{i}$ 's are odd in $\bar{\xi}$ for every $i$. It remains to compute the $L^{2}$ product of the right-hand side against $Z_{0}$. We claim that

$$
\int_{\mathbb{R}_{+}^{N}}\left(H_{\alpha \alpha} \partial_{N} w_{0}-2 H_{i j} \xi_{N} \partial_{i j}^{2} w_{0}\right) Z_{0}=\mathfrak{A}_{0} H_{\alpha \alpha}-\mathfrak{A}_{1} H_{i i}
$$

where $\mathfrak{A}_{0}$ and $\mathfrak{A}_{1}$ are the constants defined by

$$
\begin{gathered}
\mathfrak{A}_{0}=\frac{1}{2} \int_{\mathbb{R}_{+}^{N}} \xi_{N}\left|\nabla w_{0}\right|^{2}-\frac{N-2}{2 N} \int_{\mathbb{R}_{+}^{N}} \xi_{N} w_{0}^{\frac{2 N}{N-2}}, \\
\mathfrak{A}_{1}=\int_{\mathbb{R}_{+}^{N}} \xi_{N}\left|\partial_{1} w_{0}\right|^{2}>0 .
\end{gathered}
$$

Furthermore, we have

$$
\int_{\mathbb{R}_{+}^{N}} w_{0} Z_{0}=-\int_{\mathbb{R}_{+}^{N}} w_{0}^{2}
$$

Indeed, since $\left(\partial_{\mu} w_{\mu}\right)_{\mid \mu=1}=-Z_{0}$, we have that

$$
\int_{\mathbb{R}_{+}^{N}} w_{0} Z_{0}=-\int_{\mathbb{R}_{+}^{N}} w_{0}\left(\partial_{\mu} w_{\mu}\right)_{\mid \mu=1}=-\frac{1}{2} \partial_{\mu}\left(\int_{\mathbb{R}_{+}^{N}} w_{\mu}^{2}\right)_{\mid \mu=1}=-\frac{1}{2} \partial_{\mu}\left(\mu^{2} \int_{\mathbb{R}_{+}^{N}} w_{0}^{2}\right)_{\mid \mu=1} .
$$


We postpone the proof of (4.11). We turn now to the solvability in $w_{1, \varepsilon}$. Assuming the quantity on the right hand side of (4.11) is negative, we define

$$
\mu_{0}(y):=\frac{\left[\mathfrak{A}_{0} H_{\alpha \alpha}-\mathfrak{A}_{1} H_{i i}\right]}{\int_{\mathbb{R}_{+}^{N}} w_{0}^{2}} .
$$

With this choice for $\mu_{0}$, the integral of the right hand side in (4.9) against $Z_{0}$ vanishes on $K$ and this implies the existence of $w_{1, \varepsilon}$, thanks to Lemma 3.4. Moreover, it is straightforward to check that

$$
\left\|g_{1, \varepsilon}\right\|_{\varepsilon, N-2, \sigma} \leq C
$$

for some $\sigma \in(0,1)$. Lemma 3.4 thus gives that

$$
\left\|D_{\xi}^{2} w_{1, \varepsilon}\right\|_{\varepsilon, N-2, \sigma}+\left\|D_{\xi} w_{1, \varepsilon}\right\|_{\varepsilon, N-3, \sigma}+\left\|w_{1, \varepsilon}\right\|_{\varepsilon, N-4, \sigma} \leq C \varepsilon
$$

and that there exists a positive constant $\beta$ (depending only on $\Omega, K$ and $N$ ) such that for any integer $\ell$ there holds

$$
\left\|\nabla_{z}^{(\ell)} w_{1, \varepsilon}(z, \cdot)\right\|_{\varepsilon, N-2, \sigma} \leq \beta C_{l} \varepsilon \quad z \in K_{\varepsilon}
$$

where $C_{l}$ depends only on $l, p, K$ and $\Omega$.

Proof of (4.11)-(4.12)-(4.13).

We first compute $\int_{\mathbb{R}_{+}^{N}} w_{0} \partial_{N} w_{0}$. To do so, for any $\mu>0$ we denote by $w_{\mu}$ the scaled function

$$
w_{\mu}(x)=\mu^{-\frac{N-2}{2}} w_{0}\left(\mu^{-1} x\right) .
$$

Since $\left(\partial_{\mu} w_{\mu}\right)_{\left.\right|_{\mu=1}}=-Z_{0}$, a direct differentiation and integration by parts gives that

$$
\int_{\mathbb{R}_{+}^{N}} Z_{0} \partial_{N} w_{0}=\partial_{\mu}\left[\frac{1}{2} \int_{\mathbb{R}_{+}^{N}} \xi_{N}\left|\nabla w_{\mu}\right|^{2}-\frac{N-2}{2 N} \xi_{N} w_{\mu}^{\frac{2 N}{N-2}}\right]_{\left.\right|_{\mu=1}} .
$$

Now changing variables $\xi \mapsto \mu \xi$ an direct computation gives

$$
\frac{1}{2} \int_{\mathbb{R}_{+}^{N}} \xi_{N}\left|\nabla w_{\mu}\right|^{2}-\frac{N-2}{2 N} \int_{\mathbb{R}_{+}^{N}} \xi_{N} w_{\mu}^{\frac{2 N}{N-2}}=\mu\left[\frac{1}{2} \int_{\mathbb{R}_{+}^{N}} \xi_{N}\left|\nabla w_{0}\right|^{2}-\frac{N-2}{2 N} \int_{\mathbb{R}_{+}^{N}} \xi_{N} w_{0}^{\frac{2 N}{N-2}}\right] .
$$

from which (4.12) follows. Next, we compute $-2 \int_{\mathbb{R}_{+}^{N}} \xi_{N} \partial_{i j}^{2} w_{0} Z_{0}$. By symmetry we have that $2 \int_{\mathbb{R}_{+}^{N}} \xi_{N} \partial_{i j}^{2} w_{0} Z_{0}=0$ if $i \neq j$. Assume then that $i=j$ is fixed and integrations by parts, a direct differentiation yields

$$
-2 \int_{\mathbb{R}_{+}^{N}} \xi_{N} \partial_{i i}^{2} w_{0} Z_{0}=2 \int_{\mathbb{R}_{+}^{N}} \xi_{N} \partial_{i} w_{0} \partial_{i} Z_{0}=-\partial_{\mu}\left[\int_{\mathbb{R}_{+}^{N}} \xi_{N}\left|\partial_{1} w_{\mu}\right|^{2}\right]_{\left.\right|_{\mu=1}}
$$


and also

$$
\int_{\mathbb{R}_{+}^{N}} \xi_{N}\left|\partial_{1} w_{\mu}\right|^{2}=\mu \int_{\mathbb{R}_{+}^{N}} \xi_{N}\left|\partial_{1} w_{0}\right|^{2} .
$$

The above facts give the validity of (4.11). Now we claim that $\mathfrak{A}_{0}=2 \mathfrak{A}_{1}$. Indeed

$$
\begin{aligned}
\int_{\mathbb{R}_{+}^{N}} \xi_{N}\left|\nabla w_{0}\right|^{2} & =\int_{\mathbb{R}_{+}^{N}} \xi_{N}\left|\partial_{N} w_{0}\right|^{2}+\int_{\mathbb{R}_{+}^{N}} \xi_{N}\left|\partial_{i} w_{0}\right|^{2} \\
& =\int_{\mathbb{R}_{+}^{N}} \xi_{N}\left|\partial_{N} w_{0}\right|^{2}+(N-1) \int_{\mathbb{R}_{+}^{N}} \xi_{N}\left|\partial_{1} w_{0}\right|^{2} \\
& =(N+1) \int_{\mathbb{R}_{+}^{N}} \xi_{N}\left|\partial_{1} w_{0}\right|^{2}=(N+1) \mathfrak{A}_{1} .
\end{aligned}
$$

Here we used the fact that

$$
\int_{\mathbb{R}_{+}^{N}} \xi_{N}\left|\partial_{N} w_{0}\right|^{2}=2 \int_{\mathbb{R}_{+}^{N}} \xi_{N}\left|\partial_{1} w_{0}\right|^{2}
$$

since

$$
\begin{aligned}
\int_{\mathbb{R}_{+}^{N}} \xi_{N}\left|\partial_{N} w_{0}\right|^{2} & =\alpha_{N}^{2} \int_{\mathbb{R}_{+}^{N}} \xi_{N}^{2} \frac{\xi_{N}}{\left(1+|\xi|^{2}\right)^{N}} \\
& =-\alpha_{N}^{2} \frac{1}{2(N-1)} \int_{\mathbb{R}_{+}^{N}} \xi_{N}^{2} \partial_{N}\left(\frac{1}{\left(1+|\xi|^{2}\right)^{N-1}}\right) \\
& =\alpha_{N}^{2} \frac{1}{(N-1)} \int_{\mathbb{R}_{+}^{N}} \frac{\xi_{N}}{\left(1+|\xi|^{2}\right)^{N-1}}
\end{aligned}
$$

and

$$
\begin{aligned}
\int_{\mathbb{R}_{+}^{N}} \xi_{N}\left|\partial_{1} w_{0}\right|^{2} & =\alpha_{N}^{2} \int_{\mathbb{R}_{+}^{N}} \xi_{N} \xi_{1} \frac{\xi_{1}}{\left(1+|\xi|^{2}\right)^{N}} \\
& =-\alpha_{N}^{2} \frac{1}{2(N-1)} \int_{\mathbb{R}_{+}^{N}} \xi_{N} \xi_{1} \partial_{1}\left(\frac{1}{\left(1+|\xi|^{2}\right)^{N-1}}\right) \\
& =\alpha_{N}^{2} \frac{1}{2(N-1)} \int_{\mathbb{R}_{+}^{N}} \frac{\xi_{N}}{\left(1+|\xi|^{2}\right)^{N-1}} .
\end{aligned}
$$

On the other hand

$$
\begin{aligned}
\int_{\mathbb{R}_{+}^{N}} \xi_{N} w_{0}^{\frac{2 N}{N-2}} & =\frac{1}{2} \int_{\mathbb{R}_{+}^{N}} \partial_{N}\left(\xi_{N}^{2}\right) w_{0}^{\frac{2 N}{N-2}} \\
& =-\frac{1}{2} \int_{\mathbb{R}_{+}^{N}} \frac{2 N}{N-2} \xi_{N}^{2} w_{0}^{\frac{N+2}{N-2}} \partial_{N} w_{0} \\
& =-\frac{N}{N-2} \int_{\mathbb{R}_{+}^{N}} \xi_{N}^{2} \partial_{N} w_{0} \underbrace{w_{0}^{\frac{N+2}{N-2}}}_{-\Delta w_{0}} \\
& =\frac{N}{N-2} \int_{\mathbb{R}_{+}^{N}} \xi_{N}^{2} \partial_{N} w_{0}\left(\partial_{N N}^{2} w_{0}+\partial_{i i} w_{0}\right)
\end{aligned}
$$


Now we use the fact that

$$
\int_{\mathbb{R}_{+}^{N}} \xi_{N}^{2} \partial_{N} w_{0} \partial_{N N}^{2} w_{0}=-2 \int_{\mathbb{R}_{+}^{N}} \xi_{N} \partial_{N} w_{0} \partial_{N} w_{0}-\int_{\mathbb{R}_{+}^{N}} \xi_{N}^{2} \partial_{N N}^{2} w_{0} \partial_{N} w_{0}
$$

which implies that

$$
\int_{\mathbb{R}_{+}^{N}} \xi_{N}^{2} \partial_{N} w_{0} \partial_{N N}^{2} w_{0}=-\int_{\mathbb{R}_{+}^{N}} \xi_{N} \partial_{N} w_{0} \partial_{N} w_{0}=-2 \mathfrak{A}_{1}
$$

Now integrating first in $\xi_{1}$ and then in $\xi_{N}$ yields

$$
\begin{aligned}
I:=\int_{\mathbb{R}_{+}^{N}} \xi_{N}^{2} \partial_{N} w_{0} \partial_{11}^{2} w_{0} & =-\int_{\mathbb{R}_{+}^{N}} \xi_{N}^{2} \partial_{N 1}^{2} w_{0} \partial_{1} w_{0} \\
& =\int_{\mathbb{R}_{+}^{N}} \partial_{1} w_{0} \partial_{N}\left(\xi_{N}^{2} \partial_{1} w_{0}\right) \\
& =\int_{\mathbb{R}_{+}^{N}} \partial_{1} w_{0}\left(2 \xi_{N} \partial_{1} w_{0}+\xi_{N}^{2} \partial_{1 N}^{2} w_{0}\right) \\
& =2 \mathfrak{A}_{1}-I .
\end{aligned}
$$

This implies that

$$
I=\mathfrak{A}_{1} \quad \text { and } \quad \int_{\mathbb{R}_{+}^{N}} \xi_{N}^{2} \partial_{N} w_{0} \partial_{i i}^{2} w_{0}=(N-1) \int_{\mathbb{R}_{+}^{N}} \xi_{N}^{2} \partial_{N} w_{0} \partial_{11}^{2} w_{0}=(N-1) \mathfrak{A}_{1} .
$$

We deduce that

$$
\int_{\mathbb{R}_{+}^{N}} \xi_{N} w_{0}^{\frac{2 N}{N-2}}=\frac{N}{N-2}\left(-2 \mathfrak{A}_{1}+(N-1) \mathfrak{A}_{1}\right)=\frac{N(N-3)}{N-2} \mathfrak{A}_{1} .
$$

Hence

$$
\begin{aligned}
\mathfrak{A}_{0} & =\frac{1}{2} \int_{\mathbb{R}_{+}^{N}} \xi_{N}\left|\nabla w_{0}\right|^{2}-\frac{N-2}{2 N} \int_{\mathbb{R}_{+}^{N}} \xi_{N} w_{0}^{\frac{2 N}{N-2}} \\
& =\frac{N+1}{2} \mathfrak{A}_{1}-\frac{(N-2)}{2 N} \frac{N(N-3)}{N-2} \mathfrak{A}_{1}=2 \mathfrak{A}_{1} .
\end{aligned}
$$

This proves the claim. In particular Equation (4.15) can be written as

$$
\mu_{0}(y):=\mathfrak{A}_{1} \frac{\left[2 H_{\alpha \alpha}-H_{i i}\right]}{\int_{\mathbb{R}_{+}^{N}} w_{0}^{2}}=\mathfrak{A}_{1} \frac{\left[2 H_{a a}+H_{i i}\right]}{\int_{\mathbb{R}_{+}^{N}} w_{0}^{2}} .
$$

- Construction of $w_{2, \varepsilon}$ : We take $I=2, \mu_{\varepsilon}=\mu_{0}+\mu_{1, \varepsilon}, \Phi_{\varepsilon}=\Phi_{1, \varepsilon}$ and $W_{2, \varepsilon}(z, \xi)=$ $w_{0}(\xi)+w_{1, \varepsilon}(z, \xi)+w_{2, \varepsilon}(z, \xi)$, where $\mu_{0}$ and $w_{1, \varepsilon}$ have already been constructed in the previous step. Computing $S\left(W_{2, \varepsilon}\right)$ (see (3.18)) we get

$$
-\Delta w_{2, \varepsilon}+\varepsilon \mu_{0}^{2} w_{2, \varepsilon}-p w_{0}^{p-1} w_{2, \varepsilon}=\varepsilon g_{2, \varepsilon}+\mathcal{E}_{2, \varepsilon}+Q_{\varepsilon}\left(w_{2, \varepsilon}\right) .
$$


In (4.19) the function $g_{2, \varepsilon}$ is given by

$$
\begin{aligned}
g_{2, \varepsilon} & =\mu_{1, \varepsilon}(y)\left[-H_{\alpha \alpha} \partial_{\xi_{N}} w_{0}+2 \xi_{N} H_{i j} \partial_{i j}^{2} w_{0}-2 \mu_{0} w_{0}\right] \\
& +\mu_{0}(y)\left[-H_{\alpha \alpha} \partial_{\xi_{N}} w_{1, \varepsilon}+2 \xi_{N} H_{i j} \partial_{i j}^{2} w_{1, \varepsilon}\right]+\varepsilon \mathfrak{G}_{2, \varepsilon}\left(\xi, z, w_{0}, \mu_{0}\right) \\
& -\varepsilon \mu_{0} \Delta_{K} \Phi_{1, \varepsilon}^{j} \partial_{j} w_{0}-\frac{\varepsilon}{3} \mu_{0} R_{m i j l}\left(\xi_{m} \Phi_{1, \varepsilon}^{l}+\xi_{l} \Phi_{1, \varepsilon}^{m}\right) \partial_{i j}^{2} w_{0} \\
& +\varepsilon \frac{2}{3} \mu_{0} R_{m s s j} \Phi_{1, \varepsilon}^{m} \partial_{j} w_{0}+\varepsilon \mu_{0}\left(\left(\tilde{g}^{\epsilon}\right)^{a b} R_{m a a j}-\Gamma_{a}^{c}\left(E_{m}\right) \Gamma_{c}^{a}\left(E_{j}\right)\right) \Phi_{1, \varepsilon}^{m} \partial_{j} w_{0} .
\end{aligned}
$$

In (4.20) $\mathfrak{G}_{2, \varepsilon}\left(\xi, z, w_{0}, w_{1, \varepsilon}, \mu_{0}\right)$ is the sum of functions of the form

$$
\mathcal{Q}\left(\mu_{0}, \partial_{a} \mu_{0}, \partial_{a}^{2} \mu_{0}\right) a(\varepsilon z) b(\xi)
$$

where $\mathcal{Q}$ denotes a quadratic function of its arguments, $a(\varepsilon z)$ is a smooth function uniformly bounded, together with its derivatives, in $\varepsilon$ as $\varepsilon \rightarrow 0$, while the function $b$ is such that

$$
\sup _{\xi}\left(1+|\xi|^{N-2}\right)|b(\xi)|<\infty .
$$

In (4.19) the term $\mathcal{E}_{2, \varepsilon}$ can be described as the sum of functions of the form

$$
\left(\varepsilon \mathcal{L}\left(\mu_{1}, \Phi_{1}\right)+\mathcal{Q}\left(\mu_{1}, \Phi_{1}\right)\right) a(\varepsilon z) b(\xi)
$$

where $\left(\mu_{1}, \Phi_{1}\right)=\left(\mu_{1, \varepsilon}, \partial_{a} \mu_{1, \varepsilon}, \partial_{a}^{2} \mu_{1, \varepsilon}, \Phi_{1, \varepsilon}, \partial_{a} \Phi_{1, \varepsilon}, \partial_{a}^{2} \Phi_{1, \varepsilon}\right), \mathcal{L}$ denotes a linear function of its arguments, $\mathcal{Q}$ denotes a quadratic function of its arguments, $a(\varepsilon z)$ is a smooth uniformly bounded function, together with its derivatives, in $\varepsilon$ as $\varepsilon \rightarrow 0$, while the function $b$ is such that

$$
\sup _{\xi}\left(1+|\xi|^{N-2}\right)|b(\xi)|<\infty .
$$

Finally the term $Q_{\varepsilon}\left(w_{2, \varepsilon}\right)$ is a sum of quadratic terms in $w_{2, \varepsilon}$ like

$$
-\left(w_{0}+w_{1, \varepsilon}+w_{2, \varepsilon}\right)^{p}+\left(w_{0}+w_{1, \varepsilon}\right)^{p}+p\left(w_{0}+w_{1, \varepsilon}\right)^{p-1} w_{2, \varepsilon}
$$

and linear terms in $w_{2, \varepsilon}$ multiplied by a term of order $\varepsilon$, like

$$
p\left(\left(w_{0}+w_{1, \varepsilon}\right)^{p-1}-w_{0}^{p-1}\right) w_{2, \varepsilon} .
$$

We will choose $w_{2, \varepsilon}$ to satisfy the following equation

$$
\begin{cases}-\Delta w_{2, \varepsilon}-p w_{0}^{p-1} w_{2, \varepsilon}+\varepsilon \mu_{0}^{2} w_{2, \varepsilon}=\varepsilon g_{2, \varepsilon}, & \text { on } \mathbb{R}_{+}^{N} \\ \frac{\partial w_{2, \varepsilon}}{\partial \xi_{N}}=0, & \text { on }\left\{\xi_{N}=0\right\} .\end{cases}
$$

Again by Lemma 3.4, the above equation is solvable if $g_{2, \varepsilon}$ is $L^{2}$-orthogonal to $Z_{j}, j=$ $0,1, \cdots, N-1$. These orthogonality conditions will define the parameters $\mu_{1, \varepsilon}$ and the normal section $\Phi_{1, \varepsilon}$. 
$\circ$ Projection onto $Z_{0}$ and choice of $\mu_{1, \varepsilon}$ : Recalling that by definition of $\mu_{0}$ one has

$$
\int_{\mathbb{R}^{N-1} \times \mathbb{R}^{+}}\left[-H_{\alpha \alpha} \partial_{\xi_{N}} w_{0}+2 \xi_{N} H_{i j} \partial_{i j}^{2} w_{0}-\mu_{0}(y) w_{0}\right] Z_{0} d \xi=0
$$

and using the fact that $w_{0}$ is an even function in $\bar{\xi}$, we have

$$
\begin{aligned}
\int_{\mathbb{R}_{+}^{N}} g_{2, \varepsilon} Z_{0} & =\mu_{0} \int_{\mathbb{R}_{+}^{N}}\left[-H_{\alpha \alpha} \partial_{\xi_{N}} w_{1, \varepsilon}+2 \xi_{N} H_{i j} \partial_{i j}^{2} w_{1, \varepsilon}\right] Z_{0} d \xi \\
& -\mu_{0} \mu_{1, \varepsilon} \int_{\mathbb{R}_{+}^{N}} w_{0} Z_{0} d \xi+\varepsilon \int_{\mathbb{R}_{+}^{N}} \mathfrak{G}_{2, \varepsilon}\left(\xi, z, w_{0}, \mu_{0}\right) Z_{0} d \xi .
\end{aligned}
$$

We observe that the term that factors like $\mu_{1, \varepsilon}$ in the definition of $g_{2, \varepsilon}$ in (4.20) goes away after the integration thanks to relation (4.22). Here and later $\mathfrak{G}_{i, \varepsilon}$ designates a quantity that may change from line to line and which is uniformly bounded in $\varepsilon$ depending smoothly on its arguments.

Then, we define $\mu_{1, \varepsilon}$ to make the above quantity zero. The above relation defines $\mu_{1, \varepsilon}$ as a smooth function of $y$ in $K$. From estimates (4.16) for $w_{1, \varepsilon}$ we get that

$$
\left\|\mu_{1, \varepsilon}\right\|_{L^{\infty}(K)}+\left\|\partial_{a} \mu_{1, \varepsilon}\right\|_{L^{\infty}(K)}+\left\|\partial_{a}^{2} \mu_{1, \varepsilon}\right\|_{L^{\infty}(K)} \leq C \varepsilon .
$$

$\circ$ Projection onto $Z_{l}$ and choice of $\Phi_{1, \varepsilon}:$ Multiplying $g_{2, \varepsilon}$ with $\partial_{l} w_{0}$, integrating over $\mathbb{R}_{+}^{N}$ and using the fact $w_{0}$ is even in the variable $\bar{\xi}$, one obtains

$$
\begin{aligned}
\left(\varepsilon \mu_{0}\right)^{-1} \int_{\mathbb{R}_{+}^{N}} g_{2, \varepsilon} \partial_{l} w_{0} & =-\Delta_{K} \Phi_{1, \varepsilon}^{j} \int_{\mathbb{R}_{+}^{N}} \partial_{j} w_{0} \partial_{l} w_{0}+\mu_{o}^{-1} \int_{\mathbb{R}_{+}^{N}} \mathfrak{G}_{2, \varepsilon}\left(\xi, z, w_{0}, \mu_{0}, w_{1, \varepsilon}\right) \partial_{l} w_{0} \\
& -\frac{1}{3} R_{m i j s} \int_{\mathbb{R}_{+}^{N}}\left(\xi_{m} \Phi_{1, \varepsilon}^{s}+\xi_{s} \Phi_{1, \varepsilon}^{m}\right) \partial_{i j}^{2} w_{0} \partial_{l} w_{0} \\
& +\left[\frac{2}{3} R_{m s s j} \Phi_{1, \varepsilon}^{m}+\left(\left(\tilde{g}^{\varepsilon}\right)^{a b} R_{m a b j}-\Gamma_{a}^{c}\left(E_{m}\right) \Gamma_{c}^{a}\left(E_{j}\right)\right) \Phi_{1, \varepsilon}^{m}\right] \int_{\mathbb{R}_{+}^{N}} \partial_{j} w_{0} \partial_{l} w_{0} .
\end{aligned}
$$

First of all, observe that by oddness in $\bar{\xi}$ we have that

$$
\int_{\mathbb{R}_{+}^{N}} \partial_{j} w_{0} \partial_{l} w_{0}=\delta_{l j} C_{0} \quad \text { with } \quad C_{0}:=\int_{\mathbb{R}_{+}^{N}}\left|\partial_{1} w_{0}\right|^{2} .
$$

On the other hand the integral $\int_{\mathbb{R}_{+}^{N}} \xi_{m} \partial_{i j}^{2} w_{0} \partial_{l} w_{0}$ is non-zero only if, either $i=j$ and $m=l$, or $i=l$ and $j=m$, or $i=m$ and $j=l$. In the latter case we have $R_{\text {mijs }}=0$ (by the antisymmetry of the curvature tensor in the first two indices). Therefore, the first term of the second line of the above formula becomes simply

$$
\begin{aligned}
\frac{1}{3} R_{\text {mijs }} \int_{\mathbb{R}_{+}^{N}} \xi_{m} \Phi_{1, \varepsilon}^{s} \partial_{i j}^{2} w_{0} \partial_{l} w_{0} & =\frac{1}{3} R_{l i i s} \Phi_{1, \varepsilon}^{s} \int_{\mathbb{R}_{+}^{N}} \xi_{l} \partial_{l} w_{0} \partial_{i i}^{2} w_{0} d \xi \\
& +\frac{1}{3} R_{j i j s} \Phi_{1, \varepsilon}^{s} \int_{\mathbb{R}_{+}^{N}} \xi_{j} \partial_{i} w_{0} \partial_{i j}^{2} w_{0} d \xi .
\end{aligned}
$$


Observe that, integrating by parts, when $l \neq i$ (otherwise $R_{l i i s}=0$ ) there holds

$$
\int_{\mathbb{R}_{+}^{N}} \xi_{l} \partial_{l} w_{0} \partial_{i i}^{2} w_{0} d \xi=-\int_{\mathbb{R}_{+}^{N}} \xi_{l} \partial_{i} w_{0} \partial_{l i}^{2} w_{0} d \xi
$$

Hence, still by the antisymmetry of the curvature tensor we are left with

$$
-\frac{2}{3} R_{i j j s} \Phi_{1, \varepsilon}^{s} \int_{\mathbb{R}_{+}^{N}} \xi_{j} \partial_{i} w_{0} \partial_{i j}^{2} w_{0} d \xi
$$

The last integral can be computed with a further integration by parts and is equal to $-\frac{1}{2} C_{0}$, so we get

$$
\frac{1}{3} C_{0} R_{i j j s} \Phi_{1, \varepsilon}^{s} .
$$

In a similar way (permuting the indices $s$ and $m$ in the above argument), one obtains

$$
\frac{1}{3} R_{s i j m} \int_{\mathbb{R}_{+}^{N}} \xi_{s} \Phi_{1, \varepsilon}^{m} \partial_{i j}^{2} w_{0} \partial_{l} w_{0}=\frac{1}{3} C_{0} \sum_{i} R_{i j j m} \Phi_{1, \varepsilon}^{m}
$$

Collecting the above computations, it holds

$$
-\frac{1}{3} R_{m i j s} \int_{\mathbb{R}_{+}^{N}}\left(\xi_{m} \Phi_{1, \varepsilon}^{s}+\xi_{s} \Phi_{1, \varepsilon}^{m}\right) \partial_{i j}^{2} w_{0} \partial_{l} w_{0}+\frac{2}{3} R_{m s s j} \Phi_{1, \varepsilon}^{m} \int_{\mathbb{R}_{+}^{N}} \partial_{j} w_{0} \partial_{l} w_{0}=0 .
$$

Hence Formula (4.24) becomes simply

$$
\begin{aligned}
\int_{\mathbb{R}_{+}^{N}} g_{2, \varepsilon} \partial_{l} w_{0} & =-\varepsilon \mu_{0} C_{0} \Delta_{K} \Phi_{1, \varepsilon}^{l}+\varepsilon \mu_{0} C_{0}\left(\left(\tilde{g}^{\epsilon}\right)^{a b} R_{\text {maal }}-\Gamma_{a}^{c}\left(E_{m}\right) \Gamma_{c}^{a}\left(E_{l}\right)\right) \Phi_{1, \varepsilon}^{m} \\
& +\varepsilon \int_{\mathbb{R}_{+}^{N}} \mathfrak{G}_{2, \varepsilon} \partial_{l} w_{0} .
\end{aligned}
$$

We then conclude that $g_{2, \varepsilon}\left(z, \xi, w_{0}, \ldots, w_{1, \varepsilon}\right)$, the right-hand side of $(4.21)$, is $L^{2}$-orthogonal to $Z_{l}(l=1, \cdots, N-1)$ if and only if $\Phi_{1, \varepsilon}$ satisfies an equation of the form

$$
\Delta_{K} \Phi_{1, \varepsilon}^{l}-\left(\left(\tilde{g}^{\varepsilon}\right)^{a b} R_{m a b j}-\Gamma_{a}^{c}\left(E_{m}\right) \Gamma_{c}^{a}\left(E_{l}\right)\right) \Phi_{1, \varepsilon}^{m}=G_{2, \varepsilon}(\varepsilon z)
$$

for some expression $G_{2, \varepsilon}$ smooth on its argument. Observe that the operator acting on $\Phi_{1, \varepsilon}$ in the left hand side is nothing but the Jacobi operator, see (2.21), which is invertible by the non-degeneracy condition on $K$. This implies the solvability of the above equation in $\Phi_{1, \varepsilon}$.

Furthermore, equation (4.41) defines $\Phi_{1, \varepsilon}$ as a smooth function on $K$, of order $\varepsilon$, more precisely we have

$$
\left\|\Phi_{1, \varepsilon}\right\|_{L^{\infty}(K)}+\left\|\partial_{a} \Phi_{1, \varepsilon}\right\|_{L^{\infty}(K)}+\left\|\partial_{a}^{2} \Phi_{1, \varepsilon}\right\|_{L^{\infty}(K)} \leq C
$$


By our choice of $\mu_{1, \varepsilon}$ and $\Phi_{1, \varepsilon}$ we have solvability of equation (4.21) in $w_{2, \varepsilon}$. Moreover, it is straightforward to check that

$$
\left|\varepsilon g_{2, \varepsilon}(\varepsilon z, \xi)\right| \leq C \varepsilon\left|\partial_{\xi_{N}} w_{1, \varepsilon}\right| \leq C \frac{\varepsilon^{\frac{3}{2}}}{(1+|\xi|)^{N-2}}
$$

Furthermore, for a given $\sigma \in(0,1)$ we have

$$
\left\|\varepsilon g_{2, \varepsilon}\right\|_{\varepsilon, N-2, \sigma} \leq C \varepsilon^{\frac{3}{2}} .
$$

Lemma 3.4 gives then that

$$
\left\|D_{\xi}^{2} w_{2, \varepsilon}\right\|_{\varepsilon, N-2, \sigma}+\left\|D_{\xi} w_{2, \varepsilon}\right\|_{\varepsilon, N-3, \sigma}+\left\|w_{2, \varepsilon}\right\|_{\varepsilon, N-4, \sigma} \leq C \varepsilon^{\frac{3}{2}}
$$

and that there exists a positive constant $\beta$ (depending only on $\Omega, K$ and $n$ ) such that for any integer $\ell$ there holds

$$
\left\|\nabla_{z}^{(\ell)} w_{2, \varepsilon}(z, \cdot)\right\|_{\varepsilon, N-2, \sigma} \leq \beta C_{l} \varepsilon^{\frac{3}{2}} \quad z \in K_{\varepsilon}
$$

where $C_{l}$ depends only on $l, p, K$ and $\Omega$.

- Expansion at an arbitrary order: We take now an arbitrary integer $I$. Let

$$
\begin{gathered}
\mu_{\varepsilon}:=\mu_{0}+\mu_{1, \varepsilon}+\cdots+\mu_{I-1, \varepsilon}+\mu_{I, \varepsilon} \\
\Phi=\Phi_{1, \varepsilon}+\cdots+\Phi_{I-1, \varepsilon}+\Phi_{I, \varepsilon}
\end{gathered}
$$

and

$$
W_{I+1, \varepsilon}=w_{0}(\xi)+w_{1, \varepsilon}(z, \xi)+\ldots+w_{I, \varepsilon}(z, \xi)+w_{I+1, \varepsilon}(z, \xi)
$$

where $\mu_{0}, \mu_{1, \varepsilon}, \cdots, \mu_{I-1, \varepsilon}, \Phi_{1, \varepsilon}, \cdots, \Phi_{I-1, \varepsilon}$ and $w_{1, \varepsilon}, . ., w_{I, \varepsilon}$ have already been constructed following an iterative scheme, as described in the previous steps of the construction.

In particular one has, for any $i=1, \ldots, I-1$

$$
\begin{gathered}
\left\|\mu_{i, \varepsilon}\right\|_{L^{\infty}(K)}+\left\|\partial_{a} \mu_{i, \varepsilon}\right\|_{L^{\infty}(K)}+\left\|\partial_{a}^{2} \mu_{i, \varepsilon}\right\|_{L^{\infty}(K)} \leq C \varepsilon^{1+\frac{i-1}{2}} \\
\left\|\Phi_{i, \varepsilon}\right\|_{L^{\infty}(K)}+\left\|\partial_{a} \Phi_{i, \varepsilon}\right\|_{L^{\infty}(K)}+\left\|\partial_{a}^{2} \Phi_{i, \varepsilon}\right\|_{L^{\infty}(K)} \leq C \varepsilon^{\frac{i-1}{2}}
\end{gathered}
$$

and, for now $i=0, \ldots, I-1$,

$$
\left\|D_{\xi}^{2} w_{i+1, \varepsilon}\right\|_{\varepsilon, N-2, \sigma}+\left\|D_{\xi} w_{i+1, \varepsilon}\right\|_{\varepsilon, N-3, \sigma}+\left\|w_{i+1, \varepsilon}\right\|_{\varepsilon, N-4, \sigma} \leq C \varepsilon^{1+\frac{i}{2}}
$$

and, for any integer $\ell$

$$
\left\|\nabla_{z}^{(\ell)} w_{i+1, \varepsilon}(z, \cdot)\right\|_{\varepsilon, N-2, \sigma} \leq \beta C_{l} \varepsilon^{1+\frac{i}{2}}, \quad z \in K_{\varepsilon} .
$$


The new triplet $\left(\mu_{I, \varepsilon}, \Phi_{I, \varepsilon}, w_{I+1, \varepsilon}\right)$ will be found reasoning as in the construction of the triplet $\left(\mu_{1, \varepsilon}, \Phi_{1, \varepsilon}, w_{2, \varepsilon}\right)$. Computing $S\left(W_{I+1, \varepsilon}\right)$ (see (3.18)) we get

$$
-\Delta w_{I+1, \varepsilon}+\varepsilon \mu_{0}^{2} w_{I+1, \varepsilon}-p w_{0}^{p-1} w_{I+1, \varepsilon}=\varepsilon g_{I+1, \varepsilon}+\mathcal{E}_{I+1, \varepsilon}+Q_{\varepsilon}\left(w_{I+1, \varepsilon}\right) .
$$

In (4.19) the function $g_{I+1, \varepsilon}$ is given by

$$
\begin{aligned}
g_{I+1, \varepsilon} & =\mu_{I, \varepsilon}(y)\left[-H_{\alpha \alpha} \partial_{\xi_{N}} w_{0}+2 \xi_{N} H_{i j} \partial_{i j}^{2} w_{0}-2 \mu_{0} w_{0}\right] \\
& +\mu_{0}(y)\left[-H_{\alpha \alpha} \partial_{\xi_{N}} w_{I, \varepsilon}+2 \xi_{N} H_{i j} \partial_{i j}^{2} w_{I, \varepsilon}\right] \\
& +\varepsilon \mathfrak{G}_{I+1, \varepsilon}\left(\xi, z, w_{0}, . ., e_{I, \varepsilon}, \mu_{0}, \ldots, \mu_{I-1, \varepsilon}, \Phi_{1, \varepsilon}, \ldots \Phi_{I-1, \varepsilon}\right) \\
& -\varepsilon \mu_{0} \Delta_{K} \Phi_{I, \varepsilon}^{j} \partial_{j} w_{0}-\frac{\varepsilon}{3} \mu_{0} R_{m i j l}\left(\xi_{m} \Phi_{I, \varepsilon}^{l}+\xi_{l} \Phi_{I, \varepsilon}^{m}\right) \partial_{i j}^{2} w_{0} \\
& +\frac{2}{3} \varepsilon \mu_{0} R_{m s s j} \Phi_{I, \varepsilon}^{m} \partial_{j} w_{0}+\mu_{0} \varepsilon\left(\left(\tilde{g}^{\varepsilon}\right)^{a b} R_{m a b j}-\Gamma_{a}^{c}\left(E_{m}\right) \Gamma_{c}^{a}\left(E_{j}\right)\right) \Phi_{I, \varepsilon}^{m} \partial_{j} w_{0} .
\end{aligned}
$$

In (4.37) $\mathfrak{G}_{I+1, \varepsilon}(\xi, z$,$) is a smooth function with$

$$
\left\|\mathfrak{G}_{I+1, \varepsilon}\right\|_{\varepsilon, N-2, \sigma} \leq C \varepsilon^{1+\frac{I}{2}}
$$

In (4.36) the term $\mathcal{E}_{I+1, \varepsilon}$ can be described as the sum of functions of the form

$$
\left(\varepsilon \mathcal{L}\left(\mu_{I}, \Phi_{I}\right)+\mathcal{Q}\left(\mu_{I}, \Phi_{I}\right)\right) a(\varepsilon z) b(\xi)
$$

where $\left(\mu_{I}, \Phi_{I}\right)=\left(\mu_{I, \varepsilon}, \partial_{a} \mu_{I, \varepsilon}, \partial_{a}^{2} \mu_{I, \varepsilon}, \Phi_{I, \varepsilon}, \partial_{a} \Phi_{I, \varepsilon}, \partial_{a}^{2} \Phi_{I, \varepsilon}\right), \mathcal{L}$ denotes a linear function of its arguments, $\mathcal{Q}$ denotes a quadratic function of its arguments, $a(\varepsilon z)$ is a smooth function uniformly bounded, together with its derivatives, in $\varepsilon$ as $\varepsilon \rightarrow 0$, while the function $b$ is such that

$$
\sup _{\xi}\left(1+|\xi|^{N-2}\right)|b(\xi)|<\infty .
$$

Finally the term $Q_{\varepsilon}\left(w_{I+1, \varepsilon}\right)$ is a sum of quadratic terms in $w_{I+1, \varepsilon}$ like

$$
\left(w_{0}+w_{1, \varepsilon}+\ldots+w_{I+1, \varepsilon}\right)^{p}-\left(w_{0}+w_{1, \varepsilon}+\ldots+w_{I+1, \varepsilon}\right)^{p}-p\left(w_{0}+w_{1, \varepsilon}+\ldots+w_{I, \varepsilon}\right)^{p-1} w_{I+1, \varepsilon}
$$

and linear terms in $w_{I+1, \varepsilon}$ multiplied by a term of order $\varepsilon^{2}$, like

$$
p\left(\left(w_{0}+w_{1, \varepsilon}\right)^{p-1}-w_{0}^{p-1}\right) w_{I+1, \varepsilon} .
$$

We define $w_{I+1, \varepsilon}$ to satisfy the following equation

$$
\begin{cases}-\Delta w_{I+1, \varepsilon}-p w_{0}^{p-1} w_{I+1, \varepsilon}+\varepsilon \mu_{0}^{2} w_{I+1, \varepsilon}=\varepsilon g_{I+1, \varepsilon}, & \text { on } \mathbb{R}_{+}^{N} \\ \frac{\partial w_{I+1, \varepsilon}}{\partial \xi_{N}}=0, & \text { on }\left\{\xi_{N}=0\right\}\end{cases}
$$


Again by Lemma 3.4, the above equation is solvable if $g_{I+1, \varepsilon}$ is $L^{2}$-orthogonal to $Z_{j}$, $j=0,1, \cdots, N-1$. These orthogonality conditions will define the parameters $\mu_{I, \varepsilon}$ and the normal section $\Phi_{I, \varepsilon}$.

$\circ$ Projection onto $Z_{0}$ and choice of $\mu_{I, \varepsilon}$ : Thanks to the definition of $\mu_{0}$ one has

$$
\begin{aligned}
\int_{\mathbb{R}_{+}^{N}} g_{I+1, \varepsilon} Z_{0} & =\mu_{0} \int_{\mathbb{R}_{+}^{N}}\left[-H_{\alpha \alpha} \partial_{\xi_{N}} w_{I, \varepsilon}+2 \xi_{N} H_{i j} \partial_{i j}^{2} w_{I, \varepsilon}\right] Z_{0} d \xi \\
& -\mu_{0} \mu_{I, \varepsilon} \int_{\mathbb{R}_{+}^{N}} w_{0} Z_{0} d \xi+\varepsilon \int_{\mathbb{R}_{+}^{N}} \mathfrak{G}_{I+1, \varepsilon}(\xi, z) Z_{0} d \xi
\end{aligned}
$$

We define $\mu_{I, \varepsilon}$ to make the above quantity zero. The above relation defines $\mu_{I, \varepsilon}$ as a smooth function of $\varepsilon z$ in $K$. From estimates (4.34) for $w_{I, \varepsilon}$ we get that

$$
\left\|\mu_{I, \varepsilon}\right\|_{L^{\infty}(K)}+\left\|\partial_{a} \mu_{I, \varepsilon}\right\|_{L^{\infty}(K)}+\left\|\partial_{a}^{2} \mu_{I, \varepsilon}\right\|_{L^{\infty}(K)} \leq C \varepsilon^{1+\frac{I-1}{2}}
$$

$\triangleright$ Projection onto $Z_{l}$ and choice of $\Phi_{I, \varepsilon}$ : Multiplying $g_{I+1, \varepsilon}$ with $\partial_{l} w_{0}$, integrating over $\mathbb{R}_{+}^{N}$ and arguing as in the construction of $\Phi_{1, \varepsilon}$, we get

$$
\begin{aligned}
\int_{\mathbb{R}_{+}^{N}} g_{I+1, \varepsilon} \partial_{l} w_{0} & =-\varepsilon \mu_{0} \Delta_{K} \Phi_{I, \varepsilon}^{l}+\varepsilon \mu_{0}\left(\left(\tilde{g}^{\varepsilon}\right)^{a b} R_{m a b l}-\Gamma_{a}^{c}\left(E_{m}\right) \Gamma_{c}^{a}\left(E_{l}\right)\right) \Phi_{I, \varepsilon}^{m} \\
& +\varepsilon \int_{\mathbb{R}_{+}^{N}} \mathfrak{G}_{I+1, \varepsilon} \partial_{l} w_{0} .
\end{aligned}
$$

We then conclude that $g_{2, \varepsilon}\left(z, \xi, w_{0}, \ldots, w_{I, \varepsilon}\right)$, the right-hand side of (4.39), is $L^{2}$-orthogonal to $Z_{l}(l=1, \cdots, N-1)$ if and only if $\Phi_{I, \varepsilon}$ satisfies an equation of the form

$$
\Delta_{K} \Phi_{I, \varepsilon}^{l}-\left(\left(\tilde{g}^{\varepsilon}\right)^{a b} R_{m a b l}-\Gamma_{a}^{c}\left(E_{m}\right) \Gamma_{c}^{a}\left(E_{l}\right)\right) \Phi_{I, \varepsilon}^{m}=G_{I+1, \varepsilon}(\varepsilon z)
$$

where $G_{I+1, \varepsilon}$ is a smooth function on $K$. Using again the non-degeneracy condition on $K$ we have solvability of the above equation in $\Phi_{I, \varepsilon}$. Furthermore, taking into account (4.38), we get

$$
\left\|\Phi_{I, \varepsilon}\right\|_{L^{\infty}(K)}+\left\|\partial_{a} \Phi_{I, \varepsilon}\right\|_{L^{\infty}(K)}+\left\|\partial_{a}^{2} \Phi_{I, \varepsilon}\right\|_{L^{\infty}(K)} \leq C \varepsilon^{1+\frac{I-1}{2}} .
$$

By our choice of $\mu_{I+1, \varepsilon}$ and $\Phi_{I+1, \varepsilon}$ we have solvability of equation (4.39) in $w_{I+1, \varepsilon}$. Moreover, it is straightforward to check that

$$
\left|\varepsilon g_{I+1, \varepsilon}(\varepsilon z, \xi)\right| \leq C \frac{\varepsilon^{1+\frac{I}{2}}}{(1+|\xi|)^{N-2}} .
$$

Furthermore, for a given $\sigma \in(0,1)$ we have

$$
\left\|\varepsilon g_{I+1, \varepsilon}\right\|_{\varepsilon, N-2, \sigma} \leq C \varepsilon^{1+\frac{I}{2}} .
$$


Lemma 3.4 gives then that

$$
\left\|D_{\xi}^{2} w_{I+1, \varepsilon}\right\|_{\varepsilon, N-2, \sigma}+\left\|D_{\xi} w_{I+1, \varepsilon}\right\|_{\varepsilon, N-3, \sigma}+\left\|w_{I+1, \varepsilon}\right\|_{\varepsilon, N-4, \sigma} \leq C \varepsilon^{1+\frac{I}{2}}
$$

and that there exists a positive constant $\beta$ (depending only on $\Omega, K$ and $n$ ) such that for any integer $\ell$ there holds

$$
\left\|\nabla_{z}^{(\ell)} w_{I+1, \varepsilon}(z, \cdot)\right\|_{\varepsilon, N-2, \sigma} \leq \beta C_{l} \varepsilon^{1+\frac{I}{2}} \quad z \in K_{\varepsilon}
$$

This concludes our construction and have the validity of Lemma 4.1.

\section{A global approximation and Expansion of a quadratic functional}

Let $\mu_{\varepsilon}(y), \Phi_{\varepsilon}(y)$ and $W_{I+1, \varepsilon}$ be the functions whose existence and properties have been established in Lemma 4.1. We define locally around $K_{\varepsilon}:=\frac{K}{\varepsilon} \subset \partial \Omega_{\varepsilon}$ in $\Omega_{\varepsilon}$ the function

$$
\begin{aligned}
& V_{\varepsilon}(z, X):=\mu_{\varepsilon}^{-\frac{N-2}{2}}(\varepsilon z) W_{I+1, \varepsilon}\left(z, \mu_{\varepsilon}^{-1}(\varepsilon z)\left(\bar{X}-\Phi_{\varepsilon}(\varepsilon z)\right), \mu_{\varepsilon}^{-1}(\varepsilon z) X_{N}\right) \times \\
& \chi_{\varepsilon}\left(\left|\left(\bar{X}-\Phi_{\varepsilon}(\varepsilon z), X_{N}\right)\right|\right)
\end{aligned}
$$

where $z \in K_{\varepsilon}$. In (5.1) the function $\chi_{\varepsilon}$ is a smooth cut-off function with

$$
\chi_{\varepsilon}(r)= \begin{cases}1, & \text { for } \quad r \in\left[0,2 \varepsilon^{-\gamma}\right] \\ 0, & \text { for } \quad r \in\left[3 \varepsilon^{-\gamma}, 4 \varepsilon^{-\gamma}\right]\end{cases}
$$

and

$$
\left|\chi_{\varepsilon}^{(l)}(r)\right| \leq C_{l} \varepsilon^{l \gamma}, \quad \text { for all } \quad l \geq 1
$$

for some $\gamma \in\left(\frac{1}{2}, 1\right)$ to be fixed later.

The function $V_{\varepsilon}$ is well defined in a small neighborhood of $K_{\varepsilon}$ inside $\Omega_{\varepsilon}$. We will look at a solution to (1.14) of the form

$$
v_{\varepsilon}=V_{\varepsilon}+\phi
$$

This translates into the fact that $\phi$ has to satisfy the non linear problem

$$
\begin{cases}-\Delta \phi+\varepsilon \phi-p V_{\varepsilon}^{p-1} \phi=S_{\varepsilon}\left(V_{\varepsilon}\right)+N_{\varepsilon}(\phi) & \text { in } \Omega_{\varepsilon} \\ \frac{\partial \phi}{\partial \nu}=0 & \text { on } \partial \Omega_{\varepsilon}\end{cases}
$$

where

$$
S_{\varepsilon}\left(V_{\varepsilon}\right)=\Delta V_{\varepsilon}-\varepsilon V_{\varepsilon}+V_{\varepsilon}^{p}
$$


and

$$
N_{\varepsilon}(\phi)=\left(V_{\varepsilon}+\phi\right)^{p}-V_{\varepsilon}^{p}-p V_{\varepsilon}^{p-1} \phi
$$

Define

$$
L_{\varepsilon}(\phi)=-\Delta \phi+\varepsilon \phi-p V_{\varepsilon}^{p-1} \phi
$$

Our strategy consists in solving the Non-Linear Problem (5.3) using a fixed point argument based on the contraction Mapping Principle. To do so, we need to establish some invertibility properties of the linear problem

$$
L_{\varepsilon}(\phi)=f \quad \text { in } \quad \Omega_{\varepsilon}, \quad \frac{\partial \phi}{\partial \nu}=0 \quad \text { on } \quad \partial \Omega_{\varepsilon},
$$

with $f \in L^{2}\left(\Omega_{\varepsilon}\right)$. We do this in two steps. First we study the above problem in a strip close to the scaled manifold $K_{\varepsilon}=\frac{K}{\varepsilon}$ in $\partial \Omega_{\varepsilon}$. Then we establish a complete theory for the problem in the whole domain $\Omega_{\varepsilon}$ : this is done in Section 7.

Let $\gamma \in\left(\frac{1}{2}, 1\right)$ be the number fixed before in (5.2) and consider

$$
\Omega_{\varepsilon, \gamma}:=\left\{x \in \Omega_{\varepsilon}: \operatorname{dist}\left(x, K_{\varepsilon}\right)<2 \varepsilon^{-\gamma}\right\}
$$

We are first interested in solving the following problem: given $f \in L^{2}\left(\Omega_{\varepsilon, \gamma}\right)$

$$
\begin{cases}-\Delta \phi+\varepsilon \phi-p V_{\varepsilon}^{p-1} \phi=f & \text { in } \Omega_{\varepsilon, \gamma} \\ \frac{\partial \phi}{\partial \nu}=0 & \text { on } \partial \Omega_{\varepsilon} \bigcap \bar{\Omega}_{\varepsilon, \gamma} \\ \phi=0 & \text { in } \partial \Omega_{\varepsilon, \gamma} \backslash \partial \Omega_{\varepsilon}\end{cases}
$$

Observe that in the region we are considering the function $V_{\varepsilon}$ is nothing but $V_{\varepsilon}=$ $\mathcal{T}_{\mu_{\varepsilon}, \Phi_{\varepsilon}}\left(W_{I+1, \varepsilon}\right)$, where $W_{I+1, \varepsilon}$ is the function whose existence and properties are proven in Lemma 4.1. For the argument in this part of our proof it is enough to take $I=3$, and for simplicity of notation we will denote by $\hat{w}$ the function $W_{I+1, \varepsilon}$ with $I=3$. Referring to (4.3) we have

$$
\hat{w}(z, \xi)=w_{0}(\xi)+\sum_{i=1}^{4} w_{i, \varepsilon}(z, \xi)
$$

where $w_{0}$ is defined by (3.2) and

$$
\left\|D_{\xi}^{2} w_{i+1, \varepsilon}\right\|_{\varepsilon, N-2, \sigma}+\left\|D_{\xi} w_{i+1, \varepsilon}\right\|_{\varepsilon, N-3, \sigma}+\left\|w_{i+1, \varepsilon}\right\|_{\varepsilon, N-4, \sigma} \leq C \varepsilon^{1+\frac{i}{2}}
$$

and, for any integer $\ell$

$$
\left\|\nabla_{z}^{(\ell)} w_{i+1, \varepsilon}(z, \cdot)\right\|_{\varepsilon, N-2, \sigma} \leq \beta C_{l} \varepsilon^{1+\frac{i}{2}} \quad z \in K_{\varepsilon}
$$

for any $i=0,1,2,3$.

We will establish a solvability theory for Problem (5.7) in Section 6. For the moment, we devote the rest of this Section to expand the quadratic functional associated to (5.7). 
Define

$$
H_{\varepsilon}^{1}=\left\{u \in H^{1}\left(\Omega_{\varepsilon, \gamma}\right): u(x)=0 \quad \text { for } \quad x \in \partial \Omega_{\varepsilon, \gamma} \backslash \partial \Omega_{\varepsilon}\right\}
$$

and the quadratic functional given by

$$
E(\phi)=\frac{1}{2} \int_{\Omega_{\varepsilon, \gamma}}\left(|\nabla \phi|^{2}+\varepsilon \phi^{2}-p V_{\varepsilon}^{p-1} \phi^{2}\right)
$$

for functions $\phi \in H_{\varepsilon}^{1}$.

Let $(z, X) \in \mathbb{R}^{k+N}$ be the local coordinates along $K_{\varepsilon}$ introduced in (3.4), with abuse of notation we will denote

$$
\phi\left(\Upsilon_{\varepsilon}(z, X)\right)=\phi(z, X)
$$

Since the original variable $(z, X) \in \mathbb{R}^{k+N}$ (see (3.4)) are only local coordinates along $K_{\varepsilon}$ we let the variable $(z, X)$ vary in the set $\mathcal{C}_{\varepsilon}$ defined by

$$
\mathcal{C}_{\varepsilon}=\left\{\left(z, \bar{X}, X_{N}\right) / \varepsilon z \in K, \quad 0<X_{N}<\varepsilon^{-\gamma}, \quad|\bar{X}|<\varepsilon^{-\gamma}\right\}
$$

We write $\mathcal{C}_{\varepsilon}=\frac{1}{\varepsilon} K \times \hat{\mathcal{C}}_{\varepsilon}$ where

$$
\hat{\mathcal{C}}_{\varepsilon}=\left\{\left(\bar{X}, X_{N}\right) / 0<X_{N}<\varepsilon^{-\gamma}, \quad|\bar{X}|<\varepsilon^{-\gamma}\right\} .
$$

Observe that $\hat{\mathcal{C}}_{\varepsilon}$ approaches, as $\varepsilon \rightarrow 0$, the half space $\mathbb{R}_{+}^{N}$.

In these new local coordinates, the energy density associated to the energy $E$ in (5.11) is given by

$$
\left[\frac{1}{2}\left(\left|\nabla_{g^{\varepsilon}} \phi\right|^{2}+\varepsilon \phi^{2}-p V_{\varepsilon}^{p-1} \phi^{2}\right)\right] \sqrt{\operatorname{det}\left(g^{\varepsilon}\right)},
$$

where $\nabla_{g^{\varepsilon}}$ denotes the gradient in the new variables and where $g^{\varepsilon}$ is the flat metric in $\mathbb{R}^{N+k}$ in the coordinates $(z, X)$.

Having the expansion of the metric coefficients, see Lemma 3.1, we are in a position to expand the energy $(5.11)$ in the new variable $(z, X)$. Precisely we have the following

Lemma 5.1. Let $(y, x) \in \mathbb{R}^{k+N}$ be the local coordinates along the submanifold $K$ introduced in (2.8), let $(z, X)$ be the expanded variables introduced in (5.12). Assume that $(z, X)$ vary in $\mathcal{C}_{\varepsilon}$ (see (5.13)). Then, the energy functional (5.11) in the new variables (5.12) is given by

$$
\begin{aligned}
E(\phi)= & \int_{K_{\varepsilon} \times \hat{\mathcal{C}}_{\varepsilon}}\left(\frac{1}{2}\left(\left|\nabla_{X} \phi\right|^{2}+\varepsilon \phi^{2}-p V_{\varepsilon}^{p-1} \phi^{2}\right)\right) \sqrt{\operatorname{det}\left(g^{\varepsilon}\right)} d z d X \\
& +\int_{K_{\varepsilon} \times \hat{\mathcal{C}}_{\varepsilon}} \frac{1}{2} \Xi_{i j}(\varepsilon z, X) \partial_{i} \phi \partial_{j} \phi \sqrt{\operatorname{det}\left(g^{\varepsilon}\right)} d z d X \\
& +\frac{1}{2} \int_{K_{\varepsilon} \times \hat{\mathcal{C}}_{\varepsilon}}\left|\nabla_{K_{\varepsilon}} \phi\right|^{2} \sqrt{\operatorname{det}\left(g^{\varepsilon}\right)} d z d X+\int_{K_{\varepsilon} \times \hat{\mathcal{C}}_{\varepsilon}} B(\phi, \phi) \sqrt{\operatorname{det}\left(g^{\varepsilon}\right)} d z d X .
\end{aligned}
$$


In the above expression, we have

$$
\Xi_{i j}(\varepsilon z, X)=2 \varepsilon H_{i j} X_{N}-\frac{\varepsilon^{2}}{3} R_{i s l j} X_{l} X_{s}
$$

we denoted by $B(\phi, \phi)$ a quadratic term in $\phi$ that can be expressed in the following form

$$
\begin{aligned}
B(\phi, \phi)= & O\left(\varepsilon^{2} X_{N}^{2}+\varepsilon^{3}|\bar{X}|^{3}+\varepsilon^{3} X_{N}|\bar{X}|^{2}+\varepsilon^{3} X_{N}^{2}|\bar{X}|\right) \partial_{i} \phi \partial_{j} \phi \\
& +\varepsilon^{2}\left|\nabla_{K_{\varepsilon}} \phi\right|^{2} O(\varepsilon|X|)+\partial_{j} \phi \partial_{\bar{a}} \phi\left(\mathcal{O}\left(\varepsilon|\bar{X}|+\varepsilon^{2} X_{N}^{2}\right)\right) .
\end{aligned}
$$

and we used the Einstein convention over repeated indices. Furthermore we use the notation $\partial_{a}=\partial_{y_{a}}$ and $\partial_{\bar{a}}=\partial_{z_{a}}$.

Proof of Lemma 5.1. Our aim is to expand

$$
\int\left[\frac{1}{2}\left(\left|\nabla_{g^{\varepsilon}} \phi\right|^{2}+\varepsilon \phi^{2}-p V_{\varepsilon}^{p-1} \phi^{2}\right)\right] \sqrt{\operatorname{det}\left(g^{\varepsilon}\right)}
$$

For simplicity we will omit the $\varepsilon$ in the notation of $g^{\varepsilon}$. Recalling our notation about repeated indices, we write $\left|\nabla_{g^{\varepsilon}} \phi\right|^{2}$ as

$$
\left|\nabla_{g^{\varepsilon}} \phi\right|^{2}=\left(g^{\varepsilon}\right)^{N N} \partial_{N} \phi \partial_{N} \phi+\left(g^{\varepsilon}\right)^{a b} \partial_{a} \phi \partial_{b} \phi+\left(g^{\varepsilon}\right)^{i j} \partial_{i} \phi \partial_{j} \phi+2\left(g^{\varepsilon}\right)^{a j} \partial_{a} \phi \partial_{j} \phi,
$$

where $\left(g^{\varepsilon}\right)^{\alpha \beta}$ represent the coefficients of the inverse of the metric $g^{\varepsilon}=\left(g_{\alpha \beta}^{\varepsilon}\right)$. Using the expansion of the metric in Lemma 3.1, we see that

$$
\begin{aligned}
\left|\nabla_{g^{\varepsilon}} \phi\right|^{2} & =\left|\partial_{N} \phi\right|^{2}+\left|\partial_{i} \phi\right|^{2}+\left(2 \varepsilon H_{i j} X_{N}-\frac{\varepsilon^{2}}{3} R_{i s l j} X_{s} X_{l}\right) \partial_{i} \phi \partial_{j} \phi+\partial_{\bar{a}} \phi \partial_{\bar{a}} \phi(1+\varepsilon|X|) \\
& +O\left(\varepsilon^{2} X_{N}^{2}+\varepsilon^{3}|X|^{3}\right) \partial_{i} \phi \partial_{j} \phi+O\left(\varepsilon X_{N}+\varepsilon^{2} O\left(|X|^{2}\right)\right) \partial_{\bar{a}} \phi \partial_{i} \phi .
\end{aligned}
$$

This together with the expansion of $\sqrt{\operatorname{det} g}$ given in Lemma 3.1, prove Lemma 5.1.

Given a function $\phi \in H_{\varepsilon}^{1}$ (see (5.10)), we write it as

$$
\phi=\left[\frac{\delta}{\mu_{\varepsilon}} \mathcal{T}_{\mu_{\varepsilon}, \Phi_{\varepsilon}}\left(Z_{0}\right)+\sum_{j=1}^{N-1} \frac{d^{j}}{\mu_{\varepsilon}} \mathcal{T}_{\mu_{\varepsilon}, \Phi_{\varepsilon}}\left(Z_{j}\right)+\frac{e}{\mu_{\varepsilon}} \mathcal{T}_{\mu_{\varepsilon}, \Phi_{\varepsilon}}(Z)\right] \bar{\chi}_{\varepsilon}+\phi^{\perp}
$$

where the expression $\mathcal{T}_{\mu_{\varepsilon}, \Phi_{\varepsilon}}(v)$ is defined in (3.7), the functions $Z_{0}$ and $Z_{j}$ are already defined in (3.19) and where $Z$ is the eigenfunction, with $\int_{\mathbb{R}^{N}} Z^{2}=1$, corresponding to the unique positive eigenvalue $\lambda_{0}$ in $L^{2}\left(\mathbb{R}^{N}\right)$ of the problem

$$
\Delta_{\mathbb{R}^{N}} \phi+p w_{0}^{p-1} \phi=\lambda_{0} \phi \quad \text { in } \quad \mathbb{R}^{N}
$$

It is worth mentioning that $Z(\xi)$ is even and it has exponential decay of order $O\left(e^{-\sqrt{\lambda_{0}}}|\xi|\right)$ at infinity. The function $\bar{\chi}_{\varepsilon}$ is a smooth cut off function defined by

$$
\bar{\chi}_{\varepsilon}(X)=\hat{\chi}_{\varepsilon}\left(\left|\left(\frac{\bar{X}-\Phi_{\varepsilon}}{\mu_{\varepsilon}}, \frac{X_{N}}{\mu_{\varepsilon}}\right)\right|\right)
$$


with $\hat{\chi}(r)=1$ for $r \in\left(0, \frac{3}{2} \varepsilon^{-\gamma}\right)$, and $\chi(r)=0$ for $r>2 \varepsilon^{-\gamma}$. Finally, in (5.19) we have that $\delta=\delta(\varepsilon z), d^{j}=d^{j}(\varepsilon z)$ and $e=e(\varepsilon z)$ are function defined in $K$ such that for all $z \in K_{\varepsilon}$

$$
\int_{\hat{\mathcal{C}}_{\varepsilon}} \phi^{\perp} \mathcal{T}_{\mu_{\varepsilon}, \Phi_{\varepsilon}}\left(Z_{0}\right) \bar{\chi}_{\varepsilon} d X=\int_{\hat{\mathcal{C}}_{\varepsilon}} \phi^{\perp} \mathcal{T}_{\mu_{\varepsilon}, \Phi_{\varepsilon}}\left(Z_{j}\right) \bar{\chi}_{\varepsilon}=\int_{\hat{\mathcal{C}}_{\varepsilon}} \phi^{\perp} \mathcal{T}_{\mu_{\varepsilon}, \Phi_{\varepsilon}}(Z) \bar{\chi}_{\varepsilon}=0
$$

We will denote by $\left(H_{\varepsilon}^{1}\right)^{\perp}$ the subspace of the functions in $H_{\varepsilon}^{1}$ that satisfy the orthogonality conditions (5.22).

A direct computation shows that

$$
\begin{gathered}
\delta(\varepsilon z)=\frac{\int \phi \mathcal{T}_{\mu_{\varepsilon}, \Phi_{\varepsilon}}\left(Z_{0}\right)}{\mu_{\varepsilon} \int Z_{0}^{2}}\left(1+O\left(\varepsilon^{2}\right)\right)+O\left(\varepsilon^{2}\right)\left(\sum_{j} d^{j}(\varepsilon z)+e(\varepsilon z)\right), \\
d^{j}(\varepsilon z)=\frac{\int \phi \mathcal{T}_{\mu_{\varepsilon}, \Phi_{\varepsilon}}\left(Z_{j}\right)}{\mu_{\varepsilon} \int Z_{j}^{2}}\left(1+O\left(\varepsilon^{2}\right)\right)+O\left(\varepsilon^{2}\right)\left(\delta(\varepsilon z)+\sum_{i \neq j} d^{i}(\varepsilon z)+e(\varepsilon z)\right),
\end{gathered}
$$

and

$$
e(\varepsilon z)=\frac{\int \phi \mathcal{T}_{\mu_{\varepsilon}, \Phi_{\varepsilon}}(Z)}{\mu_{\varepsilon} \int Z^{2}}\left(1+O\left(\varepsilon^{2}\right)\right)+O\left(\varepsilon^{2}\right)\left(\delta(\varepsilon z)+\sum_{j} d^{j}(\varepsilon z)\right) .
$$

Observe that, since $\phi \in H_{\varepsilon}^{1}$, one easily get that the functions $\delta, d^{j}$ and $e$ belong to the Hilbert space

$$
\mathcal{H}^{1}(K)=\left\{\zeta \in \mathcal{L}^{2}(K): \partial_{a} \zeta \in \mathcal{L}^{2}(K), \quad a=1, \cdots, k\right\}
$$

Thanks to the above decomposition (5.19), we have the validity of the following expansion for $E(\phi)$.

Theorem 2. Let $\gamma=1-\sigma$, for some $\sigma>0$ and small. Assume we write $\phi \in H_{\varepsilon}^{1}$ as in (5.19) and let $d=\left(d^{1}, \ldots, d^{N-1}\right)$. Then, there exists $\varepsilon_{0}>0$ such that, for all $0<\varepsilon<\varepsilon_{0}$, the following expansion holds true

$$
E(\phi)=E\left(\phi^{\perp}\right)+\varepsilon^{-k}\left[P_{\varepsilon}(\delta)+Q_{\varepsilon}(d)+R_{\varepsilon}(e)\right]+\mathcal{M}\left(\phi^{\perp}, \delta, d, e\right) .
$$

$\operatorname{In}(5.24)$

$$
P_{\varepsilon}(\delta)=P(\delta)+P_{1}(\delta)
$$

with

$$
P(\delta)=\left[\frac{A_{\varepsilon}}{2} \int_{K} \varepsilon^{2}\left|\partial_{a}\left(\delta\left(1+o\left(\varepsilon^{2}\right) \beta_{1}^{\varepsilon}(y)\right)\right)\right|^{2}+\varepsilon \frac{B}{2} \int_{K} \delta^{2}\right]
$$

with $A_{\varepsilon}$ a real number such that $\lim _{\varepsilon \rightarrow 0} A_{\varepsilon}=A:=\int_{\mathbb{R}_{+}^{N}} Z_{0}^{2}, B=-\int_{\mathbb{R}_{+}^{N}} w_{0} Z_{0}>0$ and $\beta_{1}^{\varepsilon}$ is an explicit smooth function defined on $K$ which is uniformly bounded as $\varepsilon \rightarrow 0$; 
furthermore, $P_{1}(\delta)$ is a small compact perturbation in $\mathcal{H}^{1}(K)$ whose shape is a sum of quadratic functional in $\delta$ of the form

$$
\varepsilon^{2} \int_{K} b(y)|\delta|^{2}
$$

where $b(y)$ denotes a generic explicit function, smooth and uniformly bounded, as $\varepsilon \rightarrow 0$, in $K$. In (5.24),

$$
Q_{\varepsilon}(d)=Q(d)+Q_{1}(d)
$$

with

$$
Q(d)=\frac{\varepsilon^{2}}{2} C_{\varepsilon}\left(\int_{K}\left|\partial_{a}\left(d\left(1+o\left(\varepsilon^{2}\right) \beta_{2}^{\varepsilon}(y)\right)\right)\right|^{2}+\int_{K}\left(\left(\tilde{g}^{\varepsilon}\right)^{a b} R_{m a b l}-\Gamma_{a}^{c}\left(E_{m}\right) \Gamma_{c}^{a}\left(E_{l}\right)\right) d^{m} d^{l}\right)
$$

where $C_{\varepsilon}$ is a real number such that $\lim _{\varepsilon \rightarrow 0} C_{\varepsilon}=C:=\int_{\mathbb{R}_{+}^{N}} Z_{1}^{2}, \beta_{2}^{\varepsilon}$ is an explicit smooth function defined on $K$ which is uniformly bounded as $\varepsilon \rightarrow 0$ and the terms $R_{\text {maal }}$ and $\Gamma_{a}^{c}\left(E_{m}\right)$ are smooth functions on $K$ defined respectively in (2.6) and (2.4). Furthermore, $Q_{1}(d)$ is a small compact perturbation in $\mathcal{H}^{1}(K)$ whose shape is a sum of quadratic functional in $d$ of the form

$$
\varepsilon^{3} \int_{K} b(y) d^{i} d^{j}
$$

where again $b(y)$ is a generic explicit function, smooth and uniformly bounded, as $\varepsilon \rightarrow 0$, in $K$. In (5.24),

$$
\begin{gathered}
R_{\varepsilon}(e)=R(e)+R_{1}(e) \\
R(e)=\varepsilon^{-k}\left[\frac{D_{\varepsilon}}{2}\left(\varepsilon^{2} \int_{K}\left|\partial_{a}\left(e\left(1+e^{-\frac{\lambda_{0}}{2} \varepsilon^{-\gamma}} \beta_{3}^{\varepsilon}(y)\right)\right)\right|^{2}-\lambda_{0} \int_{K} e^{2}\right)\right]
\end{gathered}
$$

with $D_{\varepsilon}$ a real number so that $\lim _{\varepsilon \rightarrow 0} D_{\varepsilon}=D:=\int_{\mathbb{R}_{+}^{N}} Z^{2}, \beta_{3}^{\varepsilon}$ an explicit smooth function in $K$, which is uniformly bounded as $\varepsilon \rightarrow 0$, and $\lambda_{0}$ the positive number defined in (5.20). Furthermore, $R_{1}$ is a small compact perturbation in $\mathcal{H}^{1}(K)$ whose shape is a sum of quadratic functional in ef the form

$$
\varepsilon^{3} \int_{K} b(y) e^{2}
$$

where again $b(y)$ is a generic explicit function, smooth and uniformly bounded, as $\varepsilon \rightarrow 0$, in K. Finally in (5.24)

$$
\mathcal{M}:\left(H_{\varepsilon}^{1}\right)^{\perp} \times\left(\mathcal{H}^{1}(K)\right)^{N+1} \rightarrow \mathbb{R}
$$

is a continuous and differentiable functional with respect to the natural topologies, homogeneous of degree 2

$$
\mathcal{M}\left(t \phi^{\perp}, t \delta, t d, t e\right)=t^{2} \mathcal{M}\left(\phi^{\perp}, \delta, d, e\right) \quad \text { for all } \quad t
$$


The derivative of $\mathcal{M}$ with respect to each one of its variable is given by a small multiple of a linear operator in $\left(\phi^{\perp}, \delta, d, e\right)$ and it satisfies

$$
\begin{gathered}
\left\|D_{\left(\phi^{\perp}, \delta, d\right)} \mathcal{M}\left(\phi_{1}^{\perp}, \delta_{1}, d_{1}, e_{1}\right)-D_{\left(\phi^{\perp}, \delta, d\right)} \mathcal{M}\left(\phi_{2}^{\perp}, \delta_{2}, d_{2}, e_{2}\right)\right\| \leq C \varepsilon^{\gamma(N-3)} \times \\
{\left[\left\|\phi_{1}^{\perp}-\phi_{2}^{\perp}\right\|+\varepsilon^{-k}\left\|\delta_{1}-\delta_{2}\right\|_{\mathcal{H}^{1}(K)}+\varepsilon^{-k}\left\|d_{1}-d_{2}\right\|_{\left(\mathcal{H}^{1}(K)\right)^{N-1}}+\varepsilon^{-k}\left\|e_{1}-e_{2}\right\|_{\mathcal{H}^{1}(K)}\right] .}
\end{gathered}
$$

Furthermore, there exists a constant $C>0$ such that

$$
\left|\mathcal{M}\left(\phi^{\perp}, \delta, d, e\right)\right| \leq C \varepsilon^{2}\left[\left\|\phi^{\perp}\right\|^{2}+\varepsilon^{-2 k}\left(\|\delta\|_{\mathcal{H}^{1}(K)}^{2}+\|d\|_{\mathcal{H}^{1}(K)}^{2}+\|e\|_{\mathcal{H}^{1}(K)}^{2}\right)\right] .
$$

We postpone the proof of Theorem 2 to Appendix 9.

\section{Solving a linear problem close to the manifold $K$}

In this Section we study the problem of finding $\phi \in H_{\varepsilon}^{1}$ (see (5.10)) solution to the linear problem (5.7) for a given $f \in L^{2}\left(\Omega_{\varepsilon, \gamma}\right)$, (see (5.6)), and we establish a-priori bounds for the solution. This section is devoted to prove this. The result is contained in the following

Theorem 3. There exist a constant $C>0$ and a sequence $\varepsilon_{l}=\varepsilon \rightarrow 0$ such that, for any $f \in L^{2}\left(\Omega_{\varepsilon, \gamma}\right)$ there exists a solution $\phi \in H_{\varepsilon}^{1}$ to Problem (5.7) such that

$$
\|\phi\|_{H_{\varepsilon}^{1}} \leq C \varepsilon^{-\max (2, k)}\|f\|_{L^{2}\left(\Omega_{\varepsilon, \gamma}\right)} .
$$

The entire section is devoted to prove Theorem 3.

Given $\phi \in H_{\varepsilon}^{1}\left(\Omega_{\varepsilon, \gamma}\right)$. As in (5.19), we have the following decomposition of $\phi$

$$
\phi=\left[\frac{\delta}{\mu_{\varepsilon}} \mathcal{T}_{\mu_{\varepsilon}, \Phi_{\varepsilon}}\left(Z_{0}\right)+\sum_{j=1}^{N-1} \frac{d^{j}}{\mu_{\varepsilon}} \mathcal{T}_{\mu_{\varepsilon}, \Phi_{\varepsilon}}\left(Z_{j}\right)+\frac{e}{\mu_{\varepsilon}} \mathcal{T}_{\mu_{\varepsilon}, \Phi_{\varepsilon}}(Z)\right] \bar{\chi}_{\varepsilon}+\phi^{\perp} .
$$

We then define the energy functional associated to Problem (5.7)

$$
\mathcal{E}:\left(H_{\varepsilon}^{1}\right)^{\perp} \times\left(\mathcal{H}^{1}(K)\right)^{N+1} \rightarrow \mathbb{R}
$$

by

$$
\mathcal{E}\left(\phi^{\perp}, \delta, d, e\right)=E(\phi)-\mathcal{L}_{f}(\phi)
$$

where $E$ is the functional in (5.11) and $\mathcal{L}_{f}(\phi)$ is the linear operator given by

$$
\mathcal{L}_{f}(\phi)=\int_{\Omega_{\varepsilon, \gamma}} f \phi
$$

Observe that

$$
\mathcal{L}_{f}(\phi)=\mathcal{L}_{f}^{1}\left(\phi^{\perp}\right)+\varepsilon^{-k}\left[\mathcal{L}_{f}^{2}(\delta)+\mathcal{L}_{f}^{3}(d)+\mathcal{L}_{f}^{4}(e)\right]
$$


where $\mathcal{L}_{f}^{1}: H_{\varepsilon}^{1} \rightarrow \mathbb{R}, \mathcal{L}_{f}^{2}, \mathcal{L}_{f}^{4}: \mathcal{H}^{1}(K) \rightarrow \mathbb{R}$ and $\mathcal{L}_{f}^{3}:\left(\mathcal{H}^{1}(K)\right)^{N-1} \rightarrow \mathbb{R}$ with

$$
\begin{gathered}
\mathcal{L}_{f}^{1}\left(\phi^{\perp}\right)=\int_{\Omega_{\varepsilon, \gamma}} f \phi^{\perp}, \quad \varepsilon^{-k} \mathcal{L}_{f}^{2}(\delta)=\int_{\Omega_{\varepsilon, \gamma}} f \frac{\delta}{\mu_{\varepsilon}} \mathcal{T}_{\mu_{\varepsilon}, \Phi_{\varepsilon}}\left(Z_{0}\right) \bar{\chi}_{\varepsilon} \\
\varepsilon^{-k} \mathcal{L}_{f}^{3}(d)=\sum_{j=1}^{N-1} \int_{\Omega_{\varepsilon, \gamma}} f \frac{d^{j}}{\mu_{\varepsilon}} \mathcal{T}_{\mu_{\varepsilon}, \bar{\Phi}_{\varepsilon}}\left(Z_{j}\right) \bar{\chi}_{\varepsilon} \quad \text { and } \quad \varepsilon^{-k} \mathcal{L}_{f}^{4}(e)=\int_{\Omega_{\varepsilon, \gamma}} f \frac{e}{\bar{\mu}_{\varepsilon}} \mathcal{T}_{\mu_{\varepsilon}, \Phi_{\varepsilon}}(Z) \bar{\chi}_{\varepsilon} .
\end{gathered}
$$

Finding a solution $\phi \in H_{\varepsilon}^{1}$ to Problem (5.7) reduces to finding a critical point $\left(\phi^{\perp}, \delta, d, e\right)$ for $\mathcal{E}$. This will be done in several steps.

Step 1. We claim that there exist $\sigma>0$ and $\varepsilon_{0}$ such that for all $\varepsilon \in\left(0, \varepsilon_{0}\right)$ and all $\phi^{\perp} \in\left(H_{\varepsilon}^{1}\right)^{\perp}$ then

$$
E\left(\phi^{\perp}\right) \geq \sigma\left\|\phi^{\perp}\right\|_{L^{2}}^{2}
$$

Using the local change of variables (3.4) and (5.12), together with the result of Lemma 5.1 , we see that, for sufficiently small $\varepsilon>0$

$$
E\left(\phi^{\perp}\right) \geq \frac{1}{4} E_{0}\left(\phi^{\perp}\right), \quad \text { with } \quad E_{0}\left(\phi^{\perp}\right)=\int_{K_{\varepsilon} \times \hat{\mathcal{C}}_{\varepsilon}}\left[\left|\nabla_{X} \phi^{\perp}\right|^{2}-p V_{\varepsilon}^{p-1} \phi^{\perp}\right] \sqrt{\operatorname{det}\left(g^{\varepsilon}\right)}
$$

for any $\phi^{\perp}=\phi^{\perp}(\varepsilon z, X)$, with $z \in K_{\varepsilon}=\frac{1}{\varepsilon} K$. The set $\hat{\mathcal{C}}_{\varepsilon}$ is defined in (5.14) and the function $V_{\varepsilon}$ is given by (5.1). We recall that $\hat{\mathcal{C}}_{\varepsilon} \rightarrow \mathbb{R}_{+}^{N}$ as $\varepsilon \rightarrow 0$.

We will establish (6.3) showing that

$$
E_{0}\left(\phi^{\perp}\right) \geq \sigma\left\|\phi^{\perp}\right\|_{L^{2}}^{2} \quad \text { for all } \quad \phi^{\perp} .
$$

To do so, we first observe that if we scale in the $z$-variable, defining $\varphi^{\perp}(y, X)=\phi^{\perp}\left(\frac{y}{\varepsilon}, X\right)$, the relation (6.4) becomes

$$
E_{0}\left(\varphi^{\perp}\right) \geq \sigma\left\|\varphi^{\perp}\right\|_{L^{2}}^{2}
$$

Thus we are led to show the validity of (6.5). We argue by contradiction, for any $n \in \mathbb{N}^{*}$, there exist $\varepsilon_{n} \rightarrow 0$ and $\varphi_{n}^{\perp} \in\left(H_{\varepsilon_{n}}^{1}\right)^{\perp}$ such that

$$
E_{0}\left(\varphi_{n}^{\perp}\right) \leq \frac{1}{n}\left\|\varphi_{n}^{\perp}\right\|_{L^{2}}^{2}
$$

Without loss of generality we can assume that the sequence $\left(\left\|\varphi_{n}^{\perp}\right\|\right)_{n}$ is bounded, as $n \rightarrow$ $\infty$. Hence, up to subsequences, we have that

$$
\varphi_{n}^{\perp} \rightarrow \varphi^{\perp} \quad \text { in } \quad H^{1}\left(K \times \mathbb{R}_{+}^{N}\right) \quad \text { and } \quad \varphi_{n}^{\perp} \rightarrow \varphi^{\perp} \quad \text { in } \quad L^{2}\left(K \times \mathbb{R}_{+}^{N}\right)
$$

Furthermore, using the estimate in (4.7) we get that

$$
\sup _{y \in K, X \in \mathbb{R}_{+}^{N}}\left|(1+|X|)^{N-4}\left[V_{\varepsilon}\left(\frac{y}{\varepsilon}, X\right)-\mu_{0}^{-\frac{N-2}{2}}(y) w_{0}\left(\frac{\bar{X}-\Phi_{0}(y)}{\mu_{0}(y)}, \frac{X_{N}}{\mu_{0}(y)}\right)\right]\right| \rightarrow 0,
$$


as $\varepsilon \rightarrow 0$, where $\mu_{0}$ and $\Phi_{0}$ are the smooth explicit function defined in (4.6) and (4.15).

Passing to the limit as $n \rightarrow \infty$ in (6.6) and applying dominated convergence Theorem, we get

$$
\int_{K \times \mathbb{R}_{+}^{N}}\left[\left|\nabla_{X} \varphi^{\perp}\right|^{2}-p\left(\mu_{0}^{-\frac{N-2}{2}}(y) w_{0}\left(\frac{\bar{X}-\Phi_{0}(y)}{\mu_{0}(y)}, \frac{X_{N}}{\mu_{0}(y)}\right)\right)^{p-1}\left(\varphi^{\perp}\right)^{2}\right] d y d X \leq 0 .
$$

Furthermore, passing to the limit in the orthogonality condition we get, for any $y \in K$

$$
\begin{gathered}
\int_{\mathbb{R}_{+}^{N}} \varphi^{\perp}(y, X) Z_{0}\left(\frac{\bar{X}-\Phi_{0}(y)}{\mu_{0}(y)}, \frac{X_{N}}{\mu_{0}(y)}\right) d X=0, \\
\int_{\mathbb{R}_{+}^{N}} \varphi^{\perp}(y, X) Z_{j}\left(\frac{\bar{X}-\Phi_{0}(y)}{\mu_{0}(y)}, \frac{X_{N}}{\mu_{0}(y)}\right) d X=0, \quad j=1, \ldots N-1
\end{gathered}
$$

and

$$
\int_{\mathbb{R}_{+}^{N}} \varphi^{\perp}(y, X) Z\left(\frac{\bar{X}-\Phi_{0}(y)}{\mu_{0}(y)}, \frac{X_{N}}{\mu_{0}(y)}\right) d X=0 .
$$

We thus get a contradiction with (6.7), since for any function $\varphi^{\perp}$ satisfying the orthogonality conditions (6.8)-(6.10) for any $y \in K$ one has

$$
\int_{K \times \mathbb{R}_{+}^{N}}\left[\left|\nabla_{X} \varphi^{\perp}\right|^{2}-p\left(\mu_{0}^{-\frac{N-2}{2}}(y) w_{0}\left(\frac{\bar{X}-\Phi_{0}(y)}{\mu_{0}(y)}, \frac{X_{N}}{\mu_{0}(y)}\right)\right)^{p-1}\left(\varphi^{\perp}\right)^{2}\right] d y d X>0
$$

(see for instance $[17,45]$ ).

Step 2. For all $\varepsilon>0$ small, the functional $P_{\varepsilon}(\delta)$ defined in (5.25) is continuous and differentiable in $\mathcal{H}^{1}(K)$; furthermore, it is strictly convex and bounded from below since

$$
P_{\varepsilon}(\delta) \geq \frac{1}{4}\left[\frac{A}{2} \varepsilon^{2} \int_{K}\left|\partial_{a} \delta\right|^{2}+\frac{B}{2} \varepsilon \int_{K} \delta^{2}\right] \geq \sigma \varepsilon^{2}\|\delta\|_{\mathcal{H}^{1}(K)}^{2}
$$

for some small but fixed $\sigma>0$. A direct consequence of these properties is that

$$
\delta \in \mathcal{H}^{1}(K) \longmapsto P_{\varepsilon}(\delta)-\mathcal{L}_{f}^{2}(\delta)
$$

has a unique minimum $\delta$, and furthermore

$$
\varepsilon^{-\frac{k}{2}}\|\delta\|_{\mathcal{H}^{1}(K)} \leq \mathcal{C} \varepsilon^{-2}\|f\|_{L^{2}\left(\Omega_{\varepsilon, \gamma}\right)}
$$

for a given positive constant $\mathcal{C}$.

Step 3. For all $\varepsilon>0$ small, the functional $Q_{\varepsilon}$ defined in (5.27) is a small perturbation in $\left(\mathcal{H}^{1}(K)\right)^{N-1}$ of the quadratic form $\varepsilon^{2} Q_{0}(d)$, defined by

$$
\varepsilon^{2} Q_{0}(d)=\frac{\varepsilon^{2}}{2} C\left[\int_{K}\left|\partial_{a} d\right|^{2}+\int_{K}\left(\left(\tilde{g}_{\varepsilon}\right)^{a b} R_{m a b l}-\Gamma_{a}^{c}\left(E_{m}\right) \Gamma_{c}^{a}\left(E_{l}\right)\right) d^{m} d^{l}\right]
$$


with $C:=\int_{\mathbb{R}_{+}^{N}} Z_{1}^{2}$ and the terms $R_{\text {maal }}$ and $\Gamma_{a}^{c}\left(E_{m}\right)$ are smooth functions on $K$ defined respectively in (2.6) and (2.4). Recall that the non-degeneracy assumption on the minimal submanifold $K$ is equivalent to the invertibility of the operator $Q_{0}(d)$.

A consequence, for each $f \in L^{2}\left(\Omega_{\varepsilon, \gamma}\right)$,

$$
d \in\left(\mathcal{H}^{1}(K)\right)^{N-1} \longrightarrow \mathbb{R}, \quad d \longmapsto Q_{\varepsilon}(d)-\mathcal{L}_{f}^{3}(d)
$$

has a unique critical point $d$, which satisfies

$$
\varepsilon^{-\frac{k}{2}}\|d\|_{\left(\mathcal{H}^{1}(K)\right)^{N-1}} \leq \widetilde{\sigma} \varepsilon^{-2}\|f\|_{L^{2}\left(\Omega_{\varepsilon, \gamma}\right)}
$$

for some proper $\widetilde{\sigma}>0$.

Step 4. Let $f \in L^{2}\left(\Omega_{\varepsilon, \gamma}\right)$ and assume that $e$ is a given (fixed) function in $\mathcal{H}^{1}(K)$. We claim that for all $\varepsilon>0$ small enough, the functional $\mathcal{G}:\left(H_{\varepsilon}^{1}\right)^{\perp} \times\left(\mathcal{H}^{1}(K)\right)^{N} \rightarrow \mathbb{R}$

$$
\left(\phi^{\perp}, \delta, d\right) \rightarrow \mathcal{E}\left(\phi^{\perp}, \delta, d, e\right)
$$

has a critical point $\left(\phi^{\perp}, \delta, d\right)$. Furthermore there exists a positive constant $C$, independent of $\varepsilon$, such that

$$
\left\|\phi^{\perp}\right\|+\varepsilon^{-\frac{k}{2}}\left[\|\delta\|_{\mathcal{H}^{1}(K)}+\|d\|_{\left(\mathcal{H}^{1}(K)\right)^{N-1}}\right] \leq C \varepsilon^{-2}\left[\|f\|_{L^{2}\left(\Omega_{\varepsilon, \gamma}\right)}+\varepsilon^{-\frac{k}{2}} \varepsilon^{2}\|e\|_{\mathcal{H}^{1}(K)}\right] .
$$

To prove the above assertion, we first consider the functional

$$
\mathcal{G}_{0}\left(\phi^{\perp}, \delta, d\right)=\mathcal{G}\left(\phi^{\perp}, \delta, d, e\right)-\mathcal{M}\left(\phi^{\perp}, \delta, d, e\right)
$$

where $\mathcal{M}$ is the functional that recollects all mixed terms, as defined in (5.24). A direct consequence of Step 1, Step 2 and Step 3 is that $\mathcal{G}_{0}$ has a critical point $\left(\phi^{\perp}=\phi^{\perp}(f), \delta=\right.$ $\delta(f), d=d(f))$, namely the system

$$
D_{\phi^{\perp}} E\left(\phi^{\perp}\right)=D_{\phi^{\perp}} \mathcal{L}_{f}^{1}\left(\phi^{\perp}\right), \quad \varepsilon^{-\frac{k}{2}} D_{\delta} P_{\varepsilon}(\delta)=D_{\delta} \mathcal{L}_{f}^{2}(\delta), \quad \varepsilon^{-\frac{k}{2}} D_{d} Q_{\varepsilon}(d)=D_{d} \mathcal{L}_{f}^{3}(d)
$$

is uniquely solvable in $\left(H_{\varepsilon}^{1}\right)^{\perp} \times\left(\mathcal{H}^{1}(K)\right)^{N}$ and furthermore

$$
\left\|\phi^{\perp}\right\|_{H_{\varepsilon}^{1}}+\varepsilon^{-\frac{k}{2}}\|\delta\|_{\mathcal{H}^{1}(K)}+\varepsilon^{-\frac{k}{2}}\|d\|_{\left(\mathcal{H}^{1}(K)\right)^{N-1}} \leq C \varepsilon^{-2}\|f\|_{L^{2}\left(\Omega_{\varepsilon, \gamma}\right)}
$$

for some constant $C>0$, independent of $\varepsilon$.

If we now consider the complete functional $\mathcal{G}$, a critical point of $\mathcal{G}$ shall satisfy the system

$$
\left\{\begin{array}{l}
D_{\phi^{\perp}} E\left(\phi^{\perp}\right)=D_{\phi^{\perp}} \mathcal{L}_{f}^{1}\left(\phi^{\perp}\right)+D_{\phi^{\perp}} \mathcal{M}\left(\phi^{\perp}, \delta, d, e\right) \\
D_{\delta} P_{\varepsilon}(\delta)=D_{\delta} \mathcal{L}_{f}^{2}(\delta)+D_{\delta} \mathcal{M}\left(\phi^{\perp}, \delta, d, e\right) \\
D_{d} Q_{\varepsilon}(d)=D_{d} \mathcal{L}_{f}^{3}(d)+D_{d} \mathcal{M}\left(\phi^{\perp}, \delta, d, e\right)
\end{array}\right.
$$


On the other hand, as we have already observed in Theorem 2, we have

$$
\begin{gathered}
\left\|D_{\left(\phi^{\perp}, \delta, d\right)} \mathcal{M}\left(\phi_{1}^{\perp}, \delta_{1}, d_{1}, e_{1}\right)-D_{\left(\phi^{\perp}, \delta, d\right)} \mathcal{M}\left(\phi_{2}^{\perp}, \delta_{2}, d_{2}, e_{2}\right)\right\| \leq C \varepsilon^{2} \times \\
{\left[\left\|\phi_{1}^{\perp}-\phi_{2}^{\perp}\right\|+\varepsilon^{-\frac{k}{2}}\left\|\delta_{1}-\delta_{2}\right\|_{\mathcal{H}^{1}(K)}+\varepsilon^{-\frac{k}{2}}\left\|d_{1}-d_{2}\right\|_{\left(\mathcal{H}^{1}(K)\right)^{N-1}}+\varepsilon^{-\frac{k}{2}}\left\|e_{1}-e_{2}\right\|_{\mathcal{H}^{1}(K)}\right]}
\end{gathered}
$$

Thus the contraction mapping Theorem guarantees the existence of a unique solution $\left(\bar{\phi}^{\perp}, \bar{\delta}, \bar{d}\right)$ to $(6.13)$ in the set

$$
\left\|\phi^{\perp}\right\|_{H_{\varepsilon}^{1}}+\varepsilon^{-\frac{k}{2}}\|\delta\|_{\mathcal{H}^{1}(K)}+\varepsilon^{-\frac{k}{2}}\|d\|_{\left(\mathcal{H}^{1}(K)\right)^{N-1}} \leq C\left[\varepsilon^{-2}\|f\|_{L^{2}\left(\Omega_{\varepsilon, \gamma}\right)}+\varepsilon^{2} \varepsilon^{-\frac{k}{2}}\|e\|_{\mathcal{H}^{1}(K)}\right] .
$$

Furthermore, the solution $\bar{\phi}^{\perp}=\bar{\phi}^{\perp}(f, e), \bar{\delta}=\bar{\delta}(f, e)$ and $\bar{d}=\bar{d}(f, e)$ depend on $e$ in a smooth and non-local way.

Step 5. Given $f \in L^{2}\left(\Omega_{\varepsilon, \gamma}\right)$, we replace the critical point $\left(\bar{\phi}^{\perp}=\bar{\phi}^{\perp}(f, e), \bar{\delta}=\bar{\delta}(f, e), \bar{d}=\right.$ $\bar{d}(f, e))$ of $\mathcal{G}$ obtained in the previous step into the functional $\mathcal{E}\left(\phi^{\perp}, \delta, d, e\right)$ thus getting a new functional depending only on $e \in \mathcal{H}^{1}(K)$, that we denote by $\mathcal{F}_{\varepsilon}(e)$, given by

$$
\begin{aligned}
\mathcal{F}_{\varepsilon}(e) & =\varepsilon^{-k}\left[R_{\varepsilon}(e)-\mathcal{L}_{f}^{4}(e)\right]+E\left(\bar{\phi}^{\perp}(e)\right)-\varepsilon^{-k} \mathcal{L}_{f}^{1}\left(\bar{\phi}^{\perp}(e)\right)+\varepsilon^{-k}\left[P_{\varepsilon}(\bar{\delta}(e))-\mathcal{L}_{f}^{2}(\bar{\delta}(e))\right] \\
& +\varepsilon^{-k}\left[Q_{\varepsilon}(\bar{d}(e))-\mathcal{L}_{f}^{3}(\bar{d}(e))\right]+\mathcal{M}\left(\bar{\phi}^{\perp}(e), \bar{\delta}(e), \bar{d}(e), e\right)
\end{aligned}
$$

The rest of the proof is devoted to show that there exists a sequence $\varepsilon=\varepsilon_{l} \rightarrow 0$ such that

$$
D_{e} \mathcal{F}_{\varepsilon}(e)=0
$$

is solvable. Using the fact that $\left(\bar{\phi}^{\perp}, \bar{\delta}, \bar{d}\right)$ is a critical point for $\mathcal{G}$ (see Step 4 for the definition), we have that

$$
D_{e} \mathcal{F}_{\varepsilon}(e)=\varepsilon^{-k} D_{e}\left[R_{\varepsilon}(e)-\mathcal{L}_{f}^{4}(e)\right]+D_{e} \mathcal{M}\left(\bar{\phi}^{\perp}(e), \bar{\delta}(e), \bar{d}(e), e\right) .
$$

Define

$$
\mathcal{L}_{\varepsilon}:=\varepsilon^{-k} D_{e} R_{\varepsilon}(e)+D_{e} \mathcal{M}\left(\bar{\phi}^{\perp}(e), \bar{\delta}(e), \bar{d}(e), e\right),
$$

regarded as self adjoint in $\mathcal{L}^{2}(K)$. The work to solve the equation $D_{e} \mathcal{F}_{\varepsilon}(e)=0$ consists in showing the existence of a sequence $\varepsilon_{l} \rightarrow 0$ such that 0 lies suitably far away from the spectrum of $\mathcal{L}_{\varepsilon_{l}}$.

We recall now that the map

$$
\left(\phi^{\perp}, \delta, d, e\right) \rightarrow D_{e} \mathcal{M}\left(\phi^{\perp}, \delta, d, e\right)
$$

is a linear operator in the variables $\phi^{\perp}, \delta, d$, while it is constant in $e$. This is contained in the result of Theorem 2. If we furthermore take into account that the terms $\bar{\phi}^{\perp}, \bar{\delta}$ and $\bar{d}$ depend smoothly and in a non-local way through $e$, we conclude that, for any $e \in \mathcal{H}^{1}(K)$,

$$
D_{e} \mathcal{M}\left(\bar{\phi}^{\perp}(e), \bar{\delta}(e), \bar{d}(e), e\right)[e]=\varepsilon^{\gamma(N-3)} \varepsilon^{-k} \int_{K}\left(\varepsilon \eta_{1}(e) \partial_{a} e+\eta_{2}(e) e\right)^{2}
$$


where $\eta_{1}$ and $\eta_{2}$ are non local operators in $e$, that are bounded, as $\varepsilon \rightarrow 0$, on bounded sets of $\mathcal{L}^{2}(K)$. Thanks to the result contained in Theorem 2 and the above observation, we conclude that the quadratic from

$$
\Upsilon_{\varepsilon}(e):=\varepsilon^{-k} D_{e} R_{\varepsilon}(e)[e]+D_{e} \mathcal{M}\left(\bar{\phi}^{\perp}(e), \bar{\delta}(e), \bar{d}(e), e\right)[e]
$$

can be described as follows

$$
\tilde{\Upsilon}_{\varepsilon}(e)=\varepsilon^{k} \Upsilon_{\varepsilon}(e)=\Upsilon_{\varepsilon}^{0}(e)-\bar{\lambda}_{0} \int_{K} e^{2}+\varepsilon \Upsilon_{\varepsilon}^{1}(e)
$$

where

$$
\Upsilon_{\varepsilon}^{0}(e)=\varepsilon^{2} \int_{K}\left(1+\varepsilon^{\gamma(N-3)} \eta_{1}(e)\right)\left|\partial_{a}\left(e\left(1+e^{-\varepsilon^{-\lambda^{\prime}}} \beta_{3}^{\varepsilon}(y)\right)\right)\right|^{2} .
$$

In the above expression $\bar{\lambda}_{0}$ is the positive number defined by

$$
\bar{\lambda}_{0}=\left(\int_{\mathbb{R}_{+}^{N}} Z_{1}^{2}\right) \lambda_{0}
$$

$\Upsilon_{e}^{1}(e)$ is a compact quadratic form in $\mathcal{H}^{1}(K), \beta_{3}^{\varepsilon}$ is a smooth and bounded (as $\varepsilon \rightarrow 0$ ) function on $K$, given by (5.30). Finally, $\eta_{1}$ is a non local operator in $e$, which is uniformly bounded, as $\varepsilon \rightarrow 0$ on bounded sets of $\mathcal{L}^{2}(K)$.

Thus, for any $\varepsilon>0$, the eigenvalues of

$$
\mathcal{L}_{\varepsilon} e=\lambda e, \quad e \in \mathcal{H}^{1}(K)
$$

are given by a sequence $\lambda_{j}(\varepsilon)$, characterized by the Courant-Fisher formulas

$$
\lambda_{j}(\varepsilon)=\sup _{\operatorname{dim}(M)=j-1} \inf _{e \in M^{\perp} \backslash\{0\}} \frac{\tilde{\Upsilon}_{\varepsilon}(e)}{\int_{K} e^{2}}=\inf _{\operatorname{dim}(M)=j} \sup _{e \in M \backslash\{0\}} \frac{\tilde{\Upsilon}_{\varepsilon}(e)}{\int_{K} e^{2}} .
$$

The proof of Theorem 3 and of the inequality (6.1) will follow then from Step 4 and formula (6.12), together with the validity of the following

Lemma 6.1. There exist a sequence $\varepsilon_{l} \rightarrow 0$ and a constant $c>0$ such that, for all $j$, we have

$$
\left|\lambda_{j}\left(\varepsilon_{l}\right)\right| \geq c \varepsilon_{l}^{k}
$$

The proof of this Lemma follows closely the proof of a related result established in [15], but we reproduce it for completeness. We shall thus devote the rest of this section to prove Lemma 6.1.

We call $\Sigma_{\varepsilon}(e)=\frac{\tilde{\Upsilon}_{\varepsilon}(e)}{\int_{K} e^{2}}$. 
For notational convenience, we let $\sigma=\varepsilon^{2}$. We are thus interested in studying the eigenvalue problem

$$
\mathcal{L}_{\sigma} \eta=\lambda \eta, \quad \eta \in \mathcal{H}^{1}(K) .
$$

With this notation and using (6.18) and (6.19) together with the fact that $\gamma(N-3)>2$ we have that

$$
\Sigma_{\sigma}(e)=\frac{\sigma \int_{K}\left(1+o(\sigma) \eta_{1}(e)\right)\left|\partial_{a}\left(e\left(1+o(\sigma) \beta_{3}^{\sigma}(y)\right)\right)\right|^{2}}{\int_{K} e^{2}}-\bar{\lambda}_{0}+\sqrt{\sigma} \frac{\Upsilon_{\sigma}^{1}(e)}{\int_{K} e^{2}}
$$

where $o(\sigma) \rightarrow 0$ as $\sigma \rightarrow 0$.

We claim that there exists a number $\delta>0$ such that for any $\sigma_{2}>0$ and for any $j \geq 1$ such that

$$
\sigma_{2}+\left|\lambda_{j}\left(\sigma_{2}\right)\right|<\delta
$$

and any $\sigma_{1}$ with $\frac{\sigma_{2}}{2}<\sigma_{1}<\sigma_{2}$, we have that

$$
\lambda_{j}\left(\sigma_{1}\right)<\lambda_{j}\left(\sigma_{2}\right)
$$

To prove the above assertion, we start observing that, since $\beta_{3}^{\sigma}$ is an explicit, smooth and bounded (as $\sigma \rightarrow 0$ ) function on $K$, as given by (5.30) and since $\eta_{1}$ is a non local operator in $e$, which is uniformly bounded, as $\sigma \rightarrow 0$ on bounded sets of $\mathcal{L}^{2}(K)$, we have that

$$
\lim _{\sigma \rightarrow 0} \frac{\int_{K}\left(1+o(\sigma) \eta_{1}(e)\right)\left|\partial_{a}\left(e\left(1+o(\sigma) \beta_{3}^{\sigma}(y)\right)\right)\right|^{2}}{\int_{K} e^{2}}=\frac{\int_{K}\left|\partial_{a} e\right|^{2}}{\int_{K} e^{2}}
$$

and

$$
\lim _{\sigma \rightarrow 0} \sqrt{\sigma} \frac{\Upsilon_{\sigma}^{1}(e)}{\int_{K} e^{2}}=0
$$

uniformly for any $e$.

Consider now two numbers $0<\sigma_{1}<\sigma_{2}$. Then for any $e$ with $\int_{K} e^{2}=1$, we have that

$$
\begin{aligned}
\sigma_{1}^{-1} \Sigma_{\sigma_{1}}(e)-\sigma_{2}^{-1} \Sigma_{\sigma_{2}}(e) & =-\bar{\lambda}_{0} \frac{\sigma_{2}-\sigma_{1}}{\sigma_{1} \sigma_{2}}+\sigma_{1}^{-1} \Upsilon_{\sigma_{1}}^{0}(e)-\sigma_{2}^{-1} \Upsilon_{\sigma_{2}}^{0}(e) \\
& +\sigma_{1}^{-\frac{1}{2}} \Upsilon_{\sigma_{1}}^{1}(e)-\sigma_{1}^{-\frac{1}{2}} \Upsilon_{\sigma_{1}}^{1}(e)
\end{aligned}
$$

A consequence of (6.24) and (6.25) is that there exists a real number $\sigma^{*}>0$ such that, for all $\sigma_{1}<\sigma_{2}<\sigma^{*}$

$$
\left|\sigma_{1}^{-1} \Upsilon_{\sigma_{1}}^{0}(e)-\sigma_{1}^{-1} \Upsilon_{\sigma_{1}}^{0}(e)\right| \leq c\left(\sigma_{2}-\sigma_{1}\right)
$$

and

$$
\left|\sigma_{1}^{-\frac{1}{2}} \Upsilon_{\sigma_{1}}^{1}(e)-\sigma_{1}^{-\frac{1}{2}} \Upsilon_{\sigma_{1}}^{1}(e)\right| \leq c \frac{\sigma_{2}-\sigma_{1}}{\sqrt{\sigma_{1} \sigma_{2}}\left(\sigma_{1}+\sigma_{2}\right)}
$$


for some constant $c$, and uniformly for any $e$ with $\int_{K} e^{2}=1$. Thus we have that, for some $\gamma_{-}, \gamma_{+}>0$

$$
\sigma_{1}^{-1} \Sigma_{\sigma_{1}}(e)+\left(\sigma_{2}-\sigma_{1}\right) \frac{\gamma_{-}}{2 \sigma_{2}^{2}} \leq \sigma_{2}^{-1} \Sigma_{\sigma_{2}}(e) \leq \sigma_{1}^{-1} \Sigma_{\sigma_{1}}(e)+\left(\sigma_{2}-\sigma_{1}\right) \frac{2 \gamma_{+}}{\sigma_{1}^{2}}
$$

for any $\sigma_{1}<\sigma_{2}<\sigma^{*}$ and any $e$ with $\int_{K} e^{2}=1$. Thus in particular we get that, there exists $\sigma^{*}$ such that for all $0<\sigma_{1}<\sigma_{2}<\sigma^{*}$ and for all $j \geq 1$

$$
\left(\sigma_{2}-\sigma_{1}\right) \frac{\gamma_{-}}{2 \sigma_{2}^{2}} \leq \sigma_{2}^{-1} \lambda_{j}\left(\sigma_{2}\right)-\sigma_{1}^{-1} \lambda_{j}\left(\sigma_{1}\right) \leq 2\left(\sigma_{2}-\sigma_{1}\right) \frac{\gamma_{+}}{\sigma_{1}^{2}}
$$

From (6.26) it follows directly that, for all $j \geq 1$, the function $\sigma \in\left(0, \sigma^{*}\right) \rightarrow \lambda_{j}(\sigma)$ is continuous. If we now assume that $\sigma_{1} \geq \frac{\sigma_{2}}{2}$, formula (6.26) gives

$$
\lambda_{j}\left(\sigma_{1}\right) \leq \lambda_{j}\left(\sigma_{2}\right)+\frac{\sigma_{1}-\sigma_{2}}{\sigma_{2}}\left[\lambda_{j}\left(\sigma_{2}\right)+\gamma \frac{\sigma_{1}}{\sigma_{2}}\right]
$$

for some $\gamma>0$. This gives the proof of (6.23).

We will find a sequence $\sigma_{l} \in\left(2^{-(l+1)}, 2^{-l}\right)$ for $l$ large as in the statement of the Lemma.

Define

$$
€=\left\{\sigma \in\left(2^{-(l+1)}, 2^{-l}\right): \operatorname{ker} \mathcal{L}_{\sigma} \neq\{0\}\right\} .
$$

If $\sigma \in \mathfrak{七}$ then for some $j, \lambda_{j}(\sigma)=0$. Choosing $l$ sufficiently large, the continuity of the function $\sigma \rightarrow \lambda_{j}(\sigma)$ together with (6.23) imply that $\lambda_{j}\left(2^{-(l+1)}\right)<0$. In other words, for all $l$ sufficienlty large

$$
\operatorname{card}(\mathbf{E}) \leq N\left(2^{-(l+1)}\right)
$$

where $N(\sigma)$ denotes the number of negative eigenvalues of problem (6.22). We next estimate $N(\sigma)$, for small values of $\sigma$. To do so, let $a>0$ be a positive constant such that $a>\bar{\lambda}_{0}$ and consider the operator

$$
\mathcal{L}_{\sigma}^{+}=-\Delta_{K}-\frac{a}{\sigma}
$$

We call $\lambda_{j}^{+}(\sigma)$ its eigenvalues. Courant-Fisher characterization of eigenvalues gives that $\lambda_{j}(\sigma) \leq \lambda_{j}^{+}(\sigma)$ for all $j$ and for all $\sigma$ small. Thus $N(\sigma) \leq N_{+}(\sigma)$, where $N_{+}(\sigma)$ is the number of negative eigenvalues of (6.29).

Denote now by $\mu_{j}$ the eigenvalues of $-\Delta_{K}$ (ordered to be non-decreasing in $j$ and counted with their multiplicity). Weyl's asymptotic formula (see for instance [11]) states that

$$
\mu_{j}=C_{K} j^{\frac{2}{k}}+o\left(j^{\frac{2}{k}}\right) \quad \text { as } \quad j \rightarrow \infty
$$

for some positive constant $C_{K}$ depending only on the dimension $k$ of $K$. Since $\lambda_{j}^{+}=$ $\mu_{j}-\frac{a}{\sigma}$, we get

$$
N_{+}(\sigma)=C \sigma^{-\frac{k}{2}}+o\left(\sigma^{-\frac{k}{2}}\right) \quad \text { as } \quad \sigma \rightarrow 0 .
$$


This fact, together with (6.28), give $\operatorname{card}(\mathrm{E}) \leq C 2^{l \frac{k}{2}}$. Hence there exist an interval $\left(a_{l}, b_{l}\right) \subset\left(2^{-(l+1)}, 2^{-l}\right)$ such that $a_{l}, b_{l} \in \mathfrak{Ł}_{l}$, and all $\sigma \in\left(a_{l}, b_{l}\right)$ with $\operatorname{Ker} \mathcal{L}_{\sigma} \neq\{0\}$ so that

$$
b_{l}-a_{l} \geq \frac{2^{-l}-2^{-(l+1)}}{\operatorname{card}\left(\mathfrak{Ł}_{l}\right)} \geq C 2^{-l\left(1+\frac{k}{2}\right)} .
$$

Let $\sigma_{l}=\frac{a_{l}+b_{l}}{2}$. We will show that this is a sequence that verifies the statement of Lemma 6.1 and the corresponding estimate (6.21). By contradiction, assume that for some $j$ we have

$$
\left|\lambda_{j}\left(\sigma_{l}\right)\right| \leq \delta \sigma_{l}^{\frac{k}{2}}
$$

for some arbitrary $\delta>0$ small. Assume first that $0<\lambda_{j}\left(\sigma_{l}\right)<\delta \sigma_{l}^{\frac{k}{2}}$. Then from (6.27) we get

$$
\lambda_{j}\left(a_{l}\right) \leq \lambda_{j}\left(\sigma_{l}\right)-\frac{\sigma_{l}-a_{l}}{\sigma_{l}}\left[\lambda_{j}\left(\sigma_{l}\right)+\gamma \frac{a_{l}}{2 \sigma_{l}}\right]
$$

and using (6.30)-(6.31) we get to

$$
\lambda_{j}\left(a_{l}\right) \leq \delta \sigma_{l}^{\frac{k}{2}}-C \frac{2^{-l\left(1+\frac{k}{2}\right)}}{2 \sigma_{l}}\left[\lambda_{j}\left(\sigma_{l}\right)+\frac{\gamma a_{l}}{2 \sigma_{l}}\right]<0,
$$

having chosen $\delta$ small. From this is follows that $\lambda_{j}(\sigma)$ must vanish at some $\sigma \in\left(a_{l}, b_{l}\right)$, but this is in contradiction with the choice of the interval $\left(a_{l}, b_{l}\right)$.

The case $-\delta \sigma_{l}^{\frac{k}{2}}<\lambda_{j}\left(\sigma_{l}\right)<0$ can be treated in a very similar way.

This concludes the proof of Lemma 6.1.

\section{Proof of the Result}

In this section, we show the existence of a solution to Problem (1.14) of the form

$$
v_{\varepsilon}=V_{\varepsilon}+\phi
$$

where $V_{\varepsilon}$ is defined in (5.1). As already observed at the end of Section 4, this reduces to find a solution $\phi$ to

$$
\begin{cases}-\Delta \phi+\varepsilon \phi-p V_{\varepsilon}^{p-1} \phi=S_{\varepsilon}\left(V_{\varepsilon}\right)+N_{\varepsilon}(\phi) & \text { in } \Omega_{\varepsilon}, \\ \frac{\partial \phi}{\partial \nu}=0 & \text { on } \partial \Omega_{\varepsilon},\end{cases}
$$

where $S_{\varepsilon}\left(V_{\varepsilon}\right)$ is defined in (5.4), and $N_{\varepsilon}(\phi)$ in (5.5).

Given the result of Lemma 4.1, a first fact is that

$$
\left\|S_{\varepsilon}\left(V_{\varepsilon}\right)\right\|_{L^{2}\left(\Omega_{\varepsilon}\right)} \leq C \varepsilon^{1+\frac{I+1}{2}}
$$

as a direct consequence of estimate (4.8). 
Define $L_{\varepsilon} \phi:=-\Delta \phi+\varepsilon \phi-p V_{\varepsilon}^{p-1} \phi$. We claim that there exist a sequence $\varepsilon_{l} \rightarrow 0$ and a positive constant $C>0$, such that, for any $f \in L^{2}\left(\Omega_{\varepsilon_{l}}\right)$, there exists a solution $\phi \in H^{1}\left(\Omega_{\varepsilon_{l}}\right)$ to the equation

$$
L_{\varepsilon_{l}} \phi=f \quad \text { in } \Omega_{\varepsilon_{l}}, \quad \frac{\partial \phi}{\partial \nu}=0 \quad \text { on } \quad \partial \Omega_{\varepsilon_{l}} .
$$

Furthermore,

$$
\|\phi\|_{H^{1}\left(\Omega_{\varepsilon_{l}}\right)} \leq C \varepsilon_{l}^{-\max \{2, k\}}\|f\|_{L^{2}\left(\Omega_{\varepsilon_{l}}\right)} .
$$

We postpone for the moment the proof of this fact and we assume its validity. For simplicity of notations we omit the dependance of $\varepsilon$ on $l$ setting $\varepsilon_{l}=\varepsilon$. Thus $\phi \in H^{1}\left(\Omega_{\varepsilon}\right)$ is a solution to (7.1) if and only if

$$
\phi=L_{\varepsilon}^{-1}\left(S_{\varepsilon}\left(V_{\varepsilon}\right)+N_{\varepsilon}(\phi)\right) .
$$

Notice that

$$
\left\|N_{\varepsilon}(\phi)\right\|_{L^{2}\left(\Omega_{\varepsilon}\right)} \leq C\left\{\begin{array}{ll}
\|\phi\|_{H^{1}\left(\Omega_{\varepsilon}\right)}^{p} & \text { for } p \leq 2, \\
\|\phi\|_{H^{1}\left(\Omega_{\varepsilon}\right)}^{2} & \text { for } p>2
\end{array} \quad\|\phi\|_{H^{1}\left(\Omega_{\varepsilon}\right)} \leq 1\right.
$$

and

$$
\begin{aligned}
& \left\|N_{\varepsilon}\left(\phi_{1}\right)-N_{\varepsilon}\left(\phi_{2}\right)\right\|_{L^{2}\left(\Omega_{\varepsilon}\right)} \\
& \leq C \begin{cases}\left(\left\|\phi_{1}\right\|_{H^{1}\left(\Omega_{\varepsilon}\right)}^{p-1}+\left\|\phi_{2}\right\|_{H^{1}\left(\Omega_{\varepsilon}\right)}^{p-1}\right)\left\|\phi_{1}-\phi_{2}\right\|_{H^{1}\left(\Omega_{\varepsilon}\right)} & \text { for } p \leq 2, \\
\left(\left\|\phi_{1}\right\|_{H^{1}\left(\Omega_{\varepsilon}\right)}+\left\|\phi_{2}\right\|_{H^{1}\left(\Omega_{\varepsilon}\right)}\right)\left\|\phi_{1}-\phi_{2}\right\|_{H^{1}\left(\Omega_{\varepsilon}\right)} & \text { for } p>2\end{cases}
\end{aligned}
$$

for any $\phi_{1}, \phi_{2}$ in $H^{1}\left(\Omega_{\varepsilon}\right)$ with $\left\|\phi_{1}\right\|_{H^{1}\left(\Omega_{\varepsilon}\right)},\left\|\phi_{2}\right\|_{H^{1}\left(\Omega_{\varepsilon}\right)} \leq 1$.

Defining $T_{\varepsilon}: H^{1}\left(\Omega_{\varepsilon}\right) \rightarrow H^{1}\left(\Omega_{\varepsilon}\right)$ as

$$
T_{\varepsilon}(\phi)=L_{\varepsilon}^{-1}\left(S_{\varepsilon}\left(V_{\varepsilon}\right)+N_{\varepsilon}(\phi)\right)
$$

we will show that $T_{\varepsilon}$ is a contraction in some small ball in $H^{1}\left(\Omega_{\varepsilon}\right)$. A direct consequence of (7.2), (7.4), (7.5) and (7.3), is that

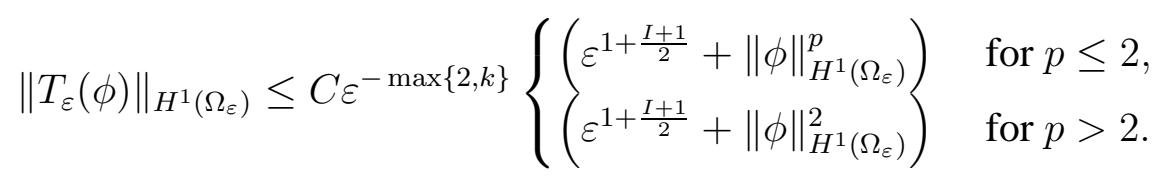

Now we choose integers $d$ and $I$ so that

$$
d>\left\{\begin{array}{ll}
\frac{\max \{2, k\}}{p-1} & \text { for } p \leq 2, \\
\max \{2, k\} & \text { for } p>2
\end{array} \quad I>d-1+\max \{2, k\} .\right.
$$

Thus one easily gets that $T_{\varepsilon}$ has a unique fixed point in set

$$
\mathcal{B}=\left\{\phi \in H^{1}\left(\Omega_{\varepsilon}\right):\|\phi\|_{H^{1}\left(\Omega_{\varepsilon}\right)} \leq \varepsilon^{d}\right\},
$$


as a direct application of the contraction mapping Theorem. This concludes the proof of Theorem 1.

We next prove the assertion previously made: we recall it. We claim that there exist a sequence $\varepsilon_{l} \rightarrow 0$ and a positive constant $\sigma>0$, such that, for any $f \in L^{2}\left(\Omega_{\varepsilon_{l}}\right)$, there exists a solution $\phi \in H^{1}\left(\Omega_{\varepsilon_{l}}\right)$ to $L_{\varepsilon_{l}} \phi=f$ in $\Omega_{\varepsilon_{l}}$, with $\frac{\partial \phi}{\partial \nu}=0$ on $\partial \Omega_{\varepsilon_{l}}$, furthermore estimate (7.3) holds true.

By contradiction, assume that for all $\varepsilon \rightarrow 0$ there exists a solution $\left(\phi_{\varepsilon}, \lambda_{\varepsilon}\right), \phi_{\varepsilon} \neq 0$, to

$$
L_{\varepsilon}\left(\phi_{\varepsilon}\right):=\Delta \phi_{\varepsilon}-\varepsilon \phi_{\varepsilon}+p V_{\varepsilon}^{p-1} \phi_{\varepsilon}=\lambda_{\varepsilon} \phi_{\varepsilon} \quad \text { in } \quad \Omega_{\varepsilon}, \quad \frac{\partial \phi_{\varepsilon}}{\partial \nu}=0 \quad \text { on } \quad \partial \Omega_{\varepsilon}
$$

with

$$
\left|\lambda_{\varepsilon}\right| \varepsilon^{-\max \{2, k\}} \rightarrow 0, \quad \text { as } \quad \varepsilon \rightarrow 0
$$

Let $\eta_{\varepsilon}$ be a smooth cut off function (like the one defined in (5.2)) so that $\eta_{\varepsilon}=1$ if dist $\left(y, K_{\varepsilon}\right)<\frac{\varepsilon^{-\gamma}}{2}$ and $\eta_{\varepsilon}=0$ if dist $\left(y, K_{\varepsilon}\right)>\varepsilon^{-\gamma}$. In particular one has that $\left|\nabla \eta_{\varepsilon}\right| \leq c \varepsilon^{\gamma}$ and $\left|\Delta \eta_{\varepsilon}\right| \leq c \varepsilon^{2 \gamma}$, in the whole domain.

Define $\tilde{\phi}_{\varepsilon}=\phi_{\varepsilon} \eta_{\varepsilon}$. Then $\tilde{\phi}_{\varepsilon}$ solves

$$
\begin{cases}L_{\varepsilon}\left(\tilde{\phi}_{\varepsilon}\right)=\lambda_{\varepsilon} \tilde{\phi}_{\varepsilon}-\nabla \eta_{\varepsilon} \nabla \phi_{\varepsilon}-\Delta \eta_{\varepsilon} \phi_{\varepsilon} & \text { in } \Omega_{\varepsilon, \gamma} \\ \frac{\partial \phi_{\varepsilon}}{\partial \nu}=0 & \text { on } \partial \Omega_{\varepsilon} \backslash \bar{\Omega}_{\varepsilon, \gamma} \\ \phi_{\varepsilon}=0 & \text { in } \partial \Omega_{\varepsilon} \cap \partial \Omega_{\varepsilon, \gamma}\end{cases}
$$

where $\Omega_{\varepsilon, \gamma}$ is the set defined in (5.6). We now apply Theorem 3, that guarantees the existence of a sequence $\varepsilon_{l} \rightarrow 0$ and a constant $c$ such that

$$
\left\|\tilde{\phi}_{\varepsilon_{l}}\right\|_{H_{\varepsilon_{l}}^{1}} \leq c \varepsilon_{l}^{-\max \{2, k\}}\left[\lambda_{\varepsilon_{l}}\left\|\tilde{\phi}_{\varepsilon_{l}}\right\|_{L^{2}}+\left\|\nabla \eta_{\varepsilon_{l}} \nabla \phi_{\varepsilon_{l}}\right\|_{L^{2}}+\left\|\Delta \eta_{\varepsilon_{l}} \phi_{\varepsilon_{l}}\right\|_{L^{2}}\right]
$$

Observe now that, in the region where $\nabla \eta_{\varepsilon_{l}} \neq 0$ and $\Delta \eta_{\varepsilon_{l}} \neq 0$, the function $V_{\varepsilon_{l}}$ can be uniformly bounded $\left|V_{\varepsilon}(y)\right| \leq c \varepsilon$, with a positive constant $c$, fact that follows directly from (5.1) and (4.7). Furthermore, since we are assuming (7.7), we see that in the region we are considering, namely where $\nabla \eta_{\varepsilon_{l}} \neq 0$ and $\Delta \eta_{\varepsilon_{l}} \neq 0$, the function $\phi_{\varepsilon_{l}}$ satisfies the equation $-\Delta \phi_{\varepsilon_{l}}+\varepsilon_{l} a_{\varepsilon_{l}}(y) \phi_{\varepsilon_{l}}=0$, for a certain smooth function $a_{\varepsilon_{l}}$, which is uniformly positive and bounded as $\varepsilon_{l} \rightarrow 0$. Elliptic estimates give that, in this region, $\left|\phi_{\varepsilon_{l}}\right| \leq c e^{-\varepsilon_{l}^{\gamma^{\prime}}}$, and $\left|\nabla \phi_{\varepsilon_{l}}\right| \leq c e^{-\varepsilon_{l}^{\gamma^{\prime}}}$ for some $\gamma^{\prime}>0$ and $c>0$. Inserting this information in (7.9), it is easy to see that

$$
\left\|\tilde{\phi}_{\varepsilon_{l}}\right\|_{H_{\varepsilon_{l}}^{1}} \leq c \varepsilon_{l}^{-\max \{2, k\}} \lambda_{\varepsilon_{l}}\left\|\tilde{\phi}_{\varepsilon_{l}}\right\|_{H_{\varepsilon_{l}}^{1}}(1+o(1))
$$

where $o(1) \rightarrow 0$ as $\varepsilon_{l} \rightarrow 0$. Taking into account (7.7) the above inequality gives a contradiction with the fact that, for all $\varepsilon$, the function $\phi_{\varepsilon}$ is not identically zero. This concludes the prove of the claim. 


\section{Appendix: Proof of Lemma 3.3}

The proof is simply based on a Taylor expansion of the metric coefficients in terms of the geometric properties of $\partial \Omega$ and $K$, as in Lemma 3.1. Recall that the Laplace-Beltrami operator is given by

$$
\Delta_{g^{\varepsilon}}=\frac{1}{\sqrt{\operatorname{det} g^{\varepsilon}}} \partial_{A}\left(\sqrt{\operatorname{det} g^{\varepsilon}}\left(g^{\varepsilon}\right)^{A B} \partial_{B}\right),
$$

where indices $A$ and $B$ run between 1 and $n=N+k$. We can write

$$
\mathcal{A}_{\mu_{\varepsilon}, \Phi_{\varepsilon}}=\left(g^{\varepsilon}\right)^{A B} \partial_{A B}^{2}+\partial_{A}\left(g^{\varepsilon}\right)^{A B} \partial_{B}+\partial_{A}\left(\log \sqrt{\operatorname{det} g^{\varepsilon}}\right)\left(g^{\varepsilon}\right)^{A B} \partial_{B} .
$$

Now, if $v$ and $W$ are defined as in (3.5), one has

$$
\begin{gathered}
\mu_{\varepsilon}^{\frac{N+2}{2}} \partial_{X_{i} X_{j}}^{2} v=\partial_{\xi_{i} \xi_{j}}^{2} W(z, \xi)=\partial_{i j}^{2} W \\
\mu_{\varepsilon}^{\frac{N+2}{2}} \partial_{X_{N}}^{2} v=\partial_{\xi_{N}}^{2} W(z, \xi)=\partial_{N N}^{2} W \\
\mu_{\varepsilon}^{\frac{N+2}{2}} \partial_{z_{a} X_{l}}^{2} v=-\frac{N}{2} \partial_{\bar{a}} \mu_{\varepsilon} \partial_{l} W+\mu_{\varepsilon} \partial_{\bar{a} l}^{2} W-\partial_{\bar{a}} \mu_{\varepsilon} \xi_{J} \partial_{l J}^{2} W-\partial_{\bar{a}} \Phi^{j} \partial_{l j}^{2} W
\end{gathered}
$$

and

$$
\begin{aligned}
\mu_{\varepsilon}^{\frac{N+2}{2}} \partial_{z_{a} z_{b}}^{2} v & =\frac{N(N-2)}{4} \partial_{\bar{a}} \mu_{\varepsilon} \partial_{\bar{b}} \mu_{\varepsilon} W-\frac{N-2}{2} \mu_{\varepsilon}\left(\partial_{\bar{a}} \mu_{\varepsilon} \partial_{\bar{b}} W+\partial_{\bar{b}} \mu_{\varepsilon} \partial_{\bar{a}} W\right) \\
& +N \partial_{\bar{a}} \mu_{\varepsilon} \partial_{\bar{b}} \mu_{\varepsilon} \xi_{J} \partial_{J} W+\frac{N}{2}\left\{\partial_{\bar{a}} \mu_{\varepsilon} \partial_{\bar{b}} \Phi^{j}+\partial_{\bar{b}} \mu_{\varepsilon} \partial_{\bar{a}} \Phi^{j}\right\} \partial_{j} W \\
& -\frac{N-2}{2} \mu_{\varepsilon} \partial_{\bar{a} \bar{b}}^{2} \mu_{\varepsilon} W+\mu_{\varepsilon}^{2} \partial_{\bar{a} \bar{b}}^{2} W-\mu_{\varepsilon} \partial_{\bar{a} \bar{b}}^{2} \mu_{\varepsilon} \xi_{J} \partial_{J} W-\mu_{\varepsilon} \partial_{\bar{a} \bar{b}}^{2} \Phi^{j} \partial_{j} W \\
& -\mu_{\varepsilon}\left(\partial_{\bar{b}} \mu_{\varepsilon} \xi_{J} \partial_{J \bar{a}}^{2} W+\partial_{\bar{a}} \mu_{\varepsilon} \xi_{J} \partial_{J \bar{b}}^{2} W\right)-\mu_{\varepsilon}\left(\partial_{\bar{b}} \Phi^{j} \partial_{j \bar{a}}^{2} W+\partial_{\bar{a}} \Phi^{j} \partial_{j \bar{b}}^{2} W\right) \\
& +\partial_{\bar{a}} \mu_{\varepsilon} \partial_{\bar{b}} \mu_{\varepsilon} \xi_{J} \xi_{L} \partial_{J L}^{2} W+\left\{\partial_{\bar{a}} \mu_{\varepsilon} \partial_{\bar{b}} \Phi^{l}+\partial_{\bar{b}} \mu_{\varepsilon} \partial_{\bar{a}} \Phi^{l}\right\} \xi_{J} \partial_{J l}^{2} W+\partial_{\bar{a}} \Phi^{l} \partial_{\bar{b}} \Phi^{j} \partial_{j l}^{2} W \\
& :=\mathcal{A}_{a b}
\end{aligned}
$$

where $\partial_{a}=\partial_{y_{a}}$ and $\partial_{\bar{a}}=\partial_{z_{a}}$ and the indices $J, L$ run between 1 and $N$ while as before the indices $j, l$ run between 1 and $N-1$.

Using the above expansions of the metric coefficients, we easily see that

$$
\begin{aligned}
& \mu_{\varepsilon}^{\frac{N+2}{2}}\left(g^{\varepsilon}\right)^{A B} \partial_{A B}^{2} v=\partial_{i i}^{2} W+\partial_{N N}^{2} W+\mu_{\varepsilon}^{2}\left(\tilde{g}^{\varepsilon}\right)^{a b} \partial_{a b}^{2} W+2 \mu_{\varepsilon} \varepsilon \xi_{N} H_{i j} \partial_{i j}^{2} W \\
& +\left\{3 \varepsilon^{2} \mu_{\varepsilon}^{2} \xi_{N}^{2}\left(H^{2}\right)_{i j}-\frac{\varepsilon^{2}}{3} R_{m i j l}\left(\mu_{\varepsilon} \xi_{l}+\Phi^{l}\right)\left(\mu_{\varepsilon} \xi_{m}+\Phi^{m}\right)\right\} \partial_{i j}^{2} W \\
& +2 \mu_{\varepsilon} \varepsilon \xi_{N}\left(H_{j a}+\left(\tilde{g}^{\varepsilon}\right)^{a c} H_{c j}\right)\left(-\frac{N-2}{2} \partial_{\bar{a}} \mu_{\varepsilon} \partial_{j} W+\frac{\mu_{\varepsilon}}{\varepsilon} \partial_{\bar{a} j}^{2} W-\partial_{\bar{a}} \mu_{\varepsilon} \xi_{L} \partial_{j L}^{2} W-\partial_{\bar{a}} \Phi^{l} \partial_{j l}^{2} W\right) \\
& +\mathcal{A}_{a a}+\mathcal{B}_{1}(W)
\end{aligned}
$$


where

$$
\begin{aligned}
\mathcal{B}_{1}(v) & =O\left(\varepsilon^{2}\left(\mu_{\varepsilon} \bar{y}+\Phi\right)^{2}+\varepsilon^{2} \mu_{\varepsilon} \xi_{N}\left(\mu_{\varepsilon} \bar{\xi}+\Phi\right)+\varepsilon^{2} \mu_{\varepsilon}^{2} \xi_{N}^{2}\right) \times \\
& \times\left(-\frac{N}{2} \partial_{\bar{a}} \mu_{\varepsilon} \partial_{l} W+\frac{\mu_{\varepsilon}}{\varepsilon} \partial_{\bar{a} l}^{2} W-\partial_{\bar{a}} \mu_{\varepsilon} \xi_{J} \partial_{l J}^{2} W-\partial_{\bar{a}} \Phi^{j} \partial_{l j}^{2} W\right) \\
& +O\left(\varepsilon^{3}\left|\mu_{\varepsilon} \bar{y}+\Phi\right|^{3}+\varepsilon^{3} \mu_{\varepsilon} \xi_{N}\left|\mu_{\varepsilon} \bar{y}+\Phi\right|^{2}+\varepsilon^{3} \mu_{\varepsilon}^{2} \xi_{N}^{2}\left|\mu_{\varepsilon} \bar{y}+\Phi\right|+\varepsilon^{3} \mu_{\varepsilon}^{3} \xi_{N}^{3}\right) \partial_{i j}^{2} W \\
& \left.+O\left(\left|\mu_{\varepsilon} \bar{y}+\Phi\right| \varepsilon+\varepsilon \mu_{\varepsilon} \xi_{N}\right)\right) \mathcal{A}_{a b} .
\end{aligned}
$$

Now recall the expansion of $\log \left(\operatorname{det} g^{\varepsilon}\right)$ given in Lemma 3.2

$$
\begin{aligned}
\log \left(\operatorname{det} g^{\varepsilon}\right) & =\log \left(\operatorname{det} \tilde{g}^{\varepsilon}\right)-2 \varepsilon X_{N} \operatorname{tr}(H)-2 \varepsilon \Gamma_{b k}^{b} X_{k}+\frac{\varepsilon^{2}}{3} R_{m i i l} x_{m} X_{l} \\
& +\varepsilon^{2}\left(\left(\tilde{g}^{\varepsilon}\right)^{a b} R_{m a b l}-\Gamma_{a m}^{c} \Gamma_{c l}^{a}\right) X_{m} X_{l}-\varepsilon^{2} X_{N}^{2} \operatorname{tr}\left(H^{2}\right)+\mathcal{O}\left(\varepsilon^{3}|X|^{3}\right) .
\end{aligned}
$$

Hence, differentiating with respect to $X_{i}, X_{N}$ and $z_{a}$ (and performing the change of variables $z=\frac{y}{\varepsilon}$ and $\bar{\xi}=\frac{\bar{X}-\Phi}{\mu_{\varepsilon}}$ and $\xi_{N}=\frac{X_{N}}{\mu_{\varepsilon}}$ ) one has

$$
\begin{aligned}
\partial_{X_{N}} \log \sqrt{\operatorname{det} g^{\varepsilon}} & =-\varepsilon \operatorname{tr}(H)-2 \mu_{\varepsilon} \varepsilon^{2} \xi_{N} \operatorname{tr}\left(H^{2}\right)+\mathcal{O}\left(\left|\left(\mu_{\varepsilon} \xi+\phi\right)\right|^{2} \varepsilon^{3}\right), \\
\partial_{X_{j}} \log \sqrt{\operatorname{det} g^{\varepsilon}} & =\varepsilon^{2}\left(\frac{1}{3} R_{m s s j}+\left(\tilde{g}^{\epsilon}\right)^{a b} R_{m a b j}-\Gamma_{a}^{c}\left(E_{m}\right) \Gamma_{c}^{a}\left(E_{j}\right)\right)\left(\mu_{\varepsilon} \xi_{m}+\Phi^{m}\right) \\
& +\mathcal{O}\left(\varepsilon^{3}\left|\left(\mu_{\varepsilon} \xi+\Phi\right)\right|^{2}\right),
\end{aligned}
$$

and

$$
\partial_{z_{a}} \log \sqrt{\operatorname{det} g^{\varepsilon}}=\varepsilon \mu_{\varepsilon} \xi_{N} \partial_{\bar{a}} \operatorname{tr}(H)+\mathcal{O}\left(\varepsilon^{2}\left|\left(\mu_{\varepsilon} \xi+\Phi\right)\right|^{2}\right)
$$

It then follows

$$
\begin{aligned}
& \mu_{\varepsilon}^{\frac{N+2}{2}} \partial_{A}\left(\log \sqrt{\operatorname{det} g^{\varepsilon}}\right) g^{A B} \partial_{B} v=\varepsilon \mu_{\varepsilon} \operatorname{tr}(H) \partial_{N} W+2 \mu_{\varepsilon} \varepsilon^{2}\left(-\mu_{\varepsilon} \xi_{N} \operatorname{tr}\left(H^{2}\right)\right) \partial_{N} W \\
& +\mu_{\varepsilon} \varepsilon^{2}\left(\frac{1}{3} R_{m s s j}\left(\mu_{\varepsilon} \xi_{m}+\Phi^{m}\right)+\left\{\left(\tilde{g}^{\varepsilon}\right)^{a b} R_{m a b j}-\Gamma_{a}^{c}\left(E_{m}\right) \Gamma_{c}^{a}\left(E_{j}\right)\right\}\left(\mu_{\varepsilon} \xi_{m}+\Phi^{m}\right)\right) \partial_{j} W \\
& +\mathcal{A}_{51}+\mathcal{B}_{2}(W)
\end{aligned}
$$

where

$$
\begin{aligned}
\mathcal{A}_{51}=-\varepsilon^{2} & \mu_{\varepsilon}^{2} \xi_{N} \partial_{a} \operatorname{tr}(H)\left(-\frac{N-2}{2} \partial_{\bar{a}} \mu_{\varepsilon} W+\mu_{\varepsilon} \partial_{\bar{a}} W-\left(\partial_{\bar{a}} \mu_{\varepsilon} \xi_{J} \partial_{J} v+\partial_{\bar{a}} \Phi^{j} \partial_{j} W\right)\right) \\
\mathcal{B}_{2}(W) & =O\left(\varepsilon^{2}\left(\mu_{\varepsilon} \bar{\xi}+\Phi\right)^{2}+\varepsilon^{2} \mu_{\varepsilon} \xi_{N}\left(\mu_{\varepsilon} \bar{\xi}+\Phi\right)+\varepsilon^{2} \mu_{\varepsilon}^{2} \xi_{N}^{2}\right)\left(\varepsilon \mu_{\varepsilon} \partial_{j} W+\varepsilon \mu_{\varepsilon} \partial_{N} W\right) \\
& +O\left(\varepsilon^{2}\left(\mu_{\varepsilon} \bar{\xi}+\Phi\right)^{2}+\varepsilon^{2} \mu_{\varepsilon} \xi_{N}\left(\mu_{\varepsilon} \bar{\xi}+\Phi\right)+\varepsilon^{2} \mu_{\varepsilon}^{2} \xi_{N}^{2}\right) \times \\
& \times\left(-\frac{N}{2} \partial_{\bar{a}} \mu_{\varepsilon} \partial_{l} W+\mu_{\varepsilon} \partial_{\bar{a} l}^{2} W-\left(\partial_{\bar{a}} \mu_{\varepsilon} \xi_{J} \partial_{l J}^{2} W+\partial_{\bar{a}} \Phi^{j} \partial_{l j}^{2} W\right)\right) .
\end{aligned}
$$


Finally, using the properties of the curvature tensor $\left(R_{i i l j}=0\right.$ and $\left.R_{s m s j}=-R_{m s s j}\right)$

$$
\begin{aligned}
\mu_{\varepsilon}^{\frac{N+2}{2}} \partial_{A}\left(g^{A B}\right) \partial_{B} v & =\partial_{i}\left(g^{i j}\right) \partial_{j} v+\partial_{a}\left(g^{a b}\right) \partial_{b} v+\partial_{a}\left(g^{a j}\right) \partial_{j} v+\partial_{j}\left(g^{a j}\right) \partial_{a} v \\
& =\frac{\varepsilon^{2}}{3} \mu_{\varepsilon} R_{l i i j}\left(\mu_{\varepsilon} \xi_{l}+\Phi^{l}\right) \partial_{j} W+\mathcal{A}_{52} W+\mathcal{B}_{3}(W)
\end{aligned}
$$

where we have set

$$
\begin{aligned}
\mathcal{A}_{52} W & =\left(\mathfrak{D}_{j}^{a}\left[\mu_{\varepsilon} \xi_{j}+\Phi^{j}\right] \varepsilon^{2}+\varepsilon \mu_{\varepsilon} \xi_{N} \mathfrak{D}_{N}^{a}\right) \times \\
& \times\left\{\mu_{\varepsilon}\left[-\varepsilon D_{\bar{\xi}} W\left[\partial_{\bar{a}} \Phi\right]+\mu_{\varepsilon} \partial_{\bar{a}} W-\varepsilon \partial_{\bar{a}} \mu_{\varepsilon}\left(\frac{N-2}{2} W+D_{\xi} W[\xi]\right)\right]\right\},
\end{aligned}
$$

where $\mathfrak{D}_{N}^{j}$ and $\mathfrak{D}_{N}^{a}$ are smooth functions on the variable $z$, and where

$$
\begin{aligned}
\mathcal{B}_{3}(W) & =O\left(\varepsilon^{2}\left(\mu_{\varepsilon} \bar{\xi}+\Phi\right)^{2}+\varepsilon^{2} \mu_{\varepsilon} \xi_{N}\left(\mu_{\varepsilon} \bar{\xi}+\Phi\right)+\varepsilon^{2} \mu_{\varepsilon}^{2} \xi_{N}^{2}\right) \mu_{\varepsilon} \varepsilon\left(\partial_{j} W+\partial_{\bar{a}} W\right) \\
& +\left(\left(\left(\mu_{\varepsilon} \bar{\xi}+\Phi\right)^{2}+\mu_{\varepsilon} \xi_{N}\left(\mu_{\varepsilon} \bar{\xi}+\Phi\right)+\mu_{\varepsilon}^{2} \xi_{N}^{2}\right) \times\right. \\
& \times\left\{\mu_{\varepsilon}\left[-\varepsilon D_{\bar{\xi}} W\left[\partial_{\bar{a}} \Phi\right]+\mu_{\varepsilon} \partial_{\bar{a}} W-\varepsilon \partial_{\bar{a}} \mu_{\varepsilon}\left(\frac{N}{2} W+D_{\xi} W[\xi]\right)\right]\right\} .
\end{aligned}
$$

Collecting these formulas together and setting

$$
\mathcal{A}_{0}=\sum_{a=1}^{k} \mathcal{A}_{a a}, \quad \mathcal{A}_{5}=\mathcal{A}_{51}+\mathcal{A}_{52},
$$

and

$$
B(v)=\mathcal{B}_{1}(v)+\mathcal{B}_{2}(v)+\mathcal{B}_{3}(v),
$$

the result follows at once.

\section{Appendix: Proof of Theorem 2}

The main ingredient to prove theorem 2 is the following

Lemma 9.1. We assume the same assumptions as in Theorem 2 and we use the same notations. Then there exists $\varepsilon_{0}>0$ such that for all $0<\varepsilon<\varepsilon_{0}$, we have

$$
\begin{aligned}
E\left(\frac{\delta}{\mu_{\varepsilon}} \mathcal{T}_{\mu_{\varepsilon}, \Phi_{\varepsilon}}\left(Z_{0}\right) \bar{\chi}_{\varepsilon}\right) & =\varepsilon^{-k} P_{\varepsilon}(\delta), \\
E\left(\frac{d^{j}}{\mu_{\varepsilon}} \mathcal{T}_{\mu_{\varepsilon}, \Phi_{\varepsilon}}\left(Z_{j}\right) \bar{\chi}_{\varepsilon}\right) & =\varepsilon^{-k} Q_{\varepsilon}\left(d^{j}\right), \\
E\left(\frac{e}{\mu_{\varepsilon}} \mathcal{T}_{\mu_{\varepsilon}, \Phi_{\varepsilon}}(Z) \bar{\chi}_{\varepsilon}\right) & =\varepsilon^{-k} R_{\varepsilon}(e) .
\end{aligned}
$$


Proof of Lemma 9.1. Define

$$
\begin{aligned}
F(u): & =\int_{K_{\varepsilon} \times \hat{\mathcal{C}}_{\varepsilon}}\left(\frac{1}{2}\left|\nabla_{X} u\right|^{2}+\frac{1}{2} \varepsilon u^{2}-\frac{1}{p+1} u^{p+1}\right) \sqrt{\operatorname{det}\left(g^{\varepsilon}\right)} d z d X \\
& +\int_{K_{\varepsilon} \times \hat{\mathcal{C}}_{\varepsilon}} \frac{1}{2} \Xi_{i j}(\varepsilon z, X) \partial_{i} u \partial_{j} u \sqrt{\operatorname{det}\left(g^{\varepsilon}\right)} d z d X \\
& +\frac{1}{2} \int_{K_{\varepsilon} \times \hat{\mathcal{C}}_{\varepsilon}} \partial_{\bar{a}} u \partial_{\bar{a}} u \sqrt{\operatorname{det}\left(g^{\varepsilon}\right)} d z d X+\int_{K_{\varepsilon} \times \hat{\mathcal{C}}_{\varepsilon}} B(u, u) \sqrt{\operatorname{det}\left(g^{\varepsilon}\right)} d z d X
\end{aligned}
$$

We refer to Lemma 5.1 for the definitions of the objects appearing in (9.4).

Step 1: Proof of (9.1). Given a small $t \neq 0$, a Taylor expansion gives that

$$
\begin{aligned}
& {\left[D F\left(\mathcal{T}_{\mu_{\varepsilon}+t \delta, \Phi_{\varepsilon}}(\hat{w}) \bar{\chi}_{\varepsilon}\right)-D F\left(\mathcal{T}_{\mu_{\varepsilon}, \bar{\Phi}_{\varepsilon}}(\hat{w}) \bar{\chi}_{\varepsilon}\right)\right]\left(\frac{\delta}{\bar{\mu}_{\varepsilon}} \mathcal{T}_{\mu_{\varepsilon}, \Phi_{\varepsilon}}\left(Z_{0}\right) \bar{\chi}_{\varepsilon}\right) } \\
= & -t D^{2} F\left(\mathcal{T}_{\mu_{\varepsilon}, \Phi_{\varepsilon}}(\hat{w}) \bar{\chi}_{\varepsilon}\right)\left[\frac{\delta}{\mu_{\varepsilon}} \mathcal{T}_{\mu_{\varepsilon}, \Phi_{\varepsilon}}\left(Z_{0}\right) \bar{\chi}_{\varepsilon}\right](1+O(t)) \\
= & -2 t E\left(\frac{\delta}{\mu_{\varepsilon}} \mathcal{T}_{\mu_{\varepsilon}, \Phi_{\varepsilon}}\left(Z_{0}\right) \bar{\chi}_{\varepsilon}\right)(1+O(t)) .
\end{aligned}
$$

On the other hand, we write for any $\psi$

$$
\left[D F\left(\mathcal{T}_{\mu_{\varepsilon}+t \delta, \bar{\Phi}_{\varepsilon}}(\hat{w}) \bar{\chi}_{\varepsilon}\right)-D F\left(\mathcal{T}_{\mu_{\varepsilon}, \Phi_{\varepsilon}}(\hat{w}) \bar{\chi}_{\varepsilon}\right)\right](\psi)=\mathfrak{a}(t)-\mathfrak{a}(0)+\mathfrak{b}(t)+\mathfrak{c}(t)
$$

where

$$
\begin{aligned}
\mathfrak{a}(t)= & \int_{K_{\varepsilon} \times \hat{\mathcal{C}}_{\varepsilon}}\left(\nabla_{X} \mathcal{T}_{\mu_{\varepsilon}+t \delta, \Phi_{\varepsilon}}(\hat{w}) \bar{\chi}_{\varepsilon}\right) \nabla_{X} \psi+\varepsilon \mathcal{T}_{\mu_{\varepsilon}+t \delta, \Phi_{\varepsilon}}(\hat{w}) \bar{\chi}_{\varepsilon} \psi-\left(\mathcal{T}_{\mu_{\varepsilon}+t \delta, \Phi_{\varepsilon}}(\hat{w}) \bar{\chi}_{\varepsilon}\right)^{p} \psi \\
+ & \int_{K_{\varepsilon} \times \hat{\mathcal{C}}_{\varepsilon}} \Xi_{i j}(\varepsilon z, X) \partial_{i}\left(\mathcal{T}_{\mu_{\varepsilon}+t \delta, \Phi_{\varepsilon}}(\hat{w}) \bar{\chi}_{\varepsilon}\right) \partial_{j} \psi \\
& \mathfrak{b}(t)=\int_{K_{\varepsilon} \times \hat{\mathcal{C}}_{\varepsilon}} \partial_{\bar{a}}\left(\mathcal{T}_{\mu_{\varepsilon}+t \delta, \Phi_{\varepsilon}}(\hat{w}) \bar{\chi}_{\varepsilon}\right) \partial_{\bar{a}} \psi-\int_{K_{\varepsilon} \times \hat{\mathcal{C}}_{\varepsilon}} \partial_{\bar{a}}\left(\mathcal{T}_{\bar{\mu}_{\varepsilon}, \Phi_{\varepsilon}}(\hat{w}) \bar{\chi}_{\varepsilon}\right) \partial_{\bar{a}} \psi
\end{aligned}
$$

and

$$
\mathfrak{c}(t)=\int_{K_{\varepsilon} \times \hat{\mathcal{C}}_{\varepsilon}} B\left(\mathcal{T}_{\bar{\mu}_{\varepsilon}+t \delta, \Phi_{\varepsilon}}(\hat{w}) \bar{\chi}_{\varepsilon}, \psi\right)-\int_{K_{\varepsilon} \times \hat{\mathcal{C}}_{\varepsilon}} B\left(\mathcal{T}_{\bar{\mu}_{\varepsilon}, \bar{\Phi}_{\varepsilon}}(\hat{w}) \bar{\chi}_{\varepsilon}, \psi\right) .
$$

We now compute $\mathfrak{a}(t)$ with $\psi=\frac{\delta}{\bar{\mu}_{\varepsilon}} \mathcal{T}_{\mu_{\varepsilon}+t \delta, \Phi_{\varepsilon}}\left(Z_{0}\right) \bar{\chi}_{\varepsilon}$. Performing the change of variables $\bar{X}=\left(\bar{\mu}_{\varepsilon}+t \delta\right) \bar{\xi}+\Phi_{\varepsilon}, X_{N}=\left(\mu_{\varepsilon}+t \delta\right) \xi_{N}$ in the integral $\mathfrak{a}(t)$ and using (3.9) together with the definition of $\bar{\chi}_{\varepsilon}$ in (5.21), we get

$$
\begin{aligned}
\mathfrak{a}(t) & =\left[-\int \frac{\delta}{\mu_{\varepsilon}}\left[\nabla \hat{w} \nabla Z_{0}+\varepsilon\left(\mu_{\varepsilon}+t \delta\right)^{2} \hat{w} Z_{0}-\hat{w}^{p} Z_{0}\right]\left(1+\varepsilon\left(\mu_{\varepsilon}+t \delta\right) \xi_{N} H_{\alpha \alpha}+\varepsilon^{2} O\left(|\xi|^{2}\right)\right)\right. \\
& \left.+-\int \frac{\delta}{\mu_{\varepsilon}}\left[-2 \varepsilon\left(\mu_{\varepsilon}+t \delta\right) \xi_{N} H_{i j}+\varepsilon^{2} O\left(|\xi|^{2}\right)\right] \partial_{i} \hat{w} \partial_{j} Z_{0}\right] \times \\
& \times(1+O(t))\left(1+O(\varepsilon)+O\left(\varepsilon^{\gamma(N-4)}\right)\right) .
\end{aligned}
$$


Thus we see immediately from (9.9) that

$$
\begin{aligned}
t^{-1}[\mathfrak{a}(t)-\mathfrak{a}(0)] & =\left[2 \varepsilon \int \delta^{2} \hat{w} Z_{0}-\int \varepsilon \frac{\delta^{2}}{\mu_{\varepsilon}}\left[\nabla \hat{w} \nabla Z_{0}+\varepsilon \mu_{\varepsilon}^{2} \hat{w} Z_{0}-\hat{w}^{p} Z_{0}\right] \xi_{N} H_{\alpha \alpha}\right. \\
& \left.+2 \varepsilon \int \frac{\delta^{2}}{\mu_{\varepsilon}} \xi_{N} H_{i j} \partial_{i} \hat{w} \partial_{j} Z_{0}\right](1+O(t))\left(1+O(\varepsilon)+O\left(\varepsilon^{\gamma(N-4)}\right)\right) .
\end{aligned}
$$

Integrating by parts in the $\xi$ variables and using the fact that $\hat{\mathcal{C}}_{\varepsilon} \rightarrow \mathbb{R}_{+}^{N}$ as $\varepsilon \rightarrow 0$ one can write

$$
\begin{gathered}
t^{-1}[\mathfrak{a}(t)-\mathfrak{a}(0)]=\left[2 \varepsilon \int \delta^{2} \hat{w} Z_{0}-\int \varepsilon \frac{\delta^{2}}{\mu_{\varepsilon}}\left[-\Delta \hat{w}+\varepsilon \mu_{\varepsilon}^{2} \hat{w}-\hat{w}^{p}\right] Z_{0} \xi_{N} H_{\alpha \alpha}\right. \\
\left.+\int \varepsilon \frac{\delta^{2}}{\mu_{\varepsilon}} \hat{w} Z_{0} H_{\alpha \alpha}-2 \varepsilon \int \frac{\delta^{2}}{\mu_{\varepsilon}} \xi_{N} H_{i j} \partial_{i j} \hat{w} Z_{0}\right](1+O(t))\left(1+O(\varepsilon)+O\left(\varepsilon^{\gamma(N-4)}\right)\right) .
\end{gathered}
$$

Now using the fact that $\left\|-\Delta \hat{w}+\varepsilon \mu_{\varepsilon}^{2} \hat{w}-\hat{w}^{p}\right\|_{\varepsilon, N-2} \leq C \varepsilon^{3}$ we get

$$
\begin{aligned}
t^{-1}[\mathfrak{a}(t)-\mathfrak{a}(0)]= & {\left[2 \varepsilon \int \delta^{2} \hat{w} Z_{0}+\varepsilon\left[\int \frac{\delta^{2}}{\mu_{0}}\left(\int_{\mathbb{R}_{+}^{N}} H_{\alpha \alpha} \hat{w} Z_{0} \xi_{N}-2 \int_{\mathbb{R}_{+}^{N}} H_{i j} \partial_{i j} \hat{w} Z_{0}\right)\right]\right.} \\
& \left.+\varepsilon^{2} Q(\delta)\right](1+O(t))\left(1+O(\varepsilon)+O\left(\varepsilon^{\gamma(N-4)}\right)\right) .
\end{aligned}
$$

Since $N>6$, we can choose $\gamma=1-\sigma, \sigma>0$ so that $\gamma(N-4)>2$. Thanks to the definition of $\mu_{0}$ given in (4.15), we conclude that

$$
\mathfrak{a}(t)-\mathfrak{a}(0)=t \varepsilon^{-k}\left[-B \varepsilon \int_{K} \delta^{2}+O\left(\varepsilon^{2}\right) Q(\delta)\right](1+O(t))(1+O(\varepsilon))
$$

where

$$
-B=\left(\int_{\mathbb{R}_{+}^{N}} w Z_{0}\right)<0 \quad \text { and } \quad Q(\delta)=\int_{K} \kappa(y) \delta^{2}
$$

for some smooth and uniformly bounded (as $\varepsilon \rightarrow 0$ ) function $\kappa$ defined on $K$.

Observe that $\partial_{\mu} \mathcal{T}_{\mu, \Phi}(\hat{w})=-\frac{1}{\mu} \mathcal{T}_{\mu, \Phi}\left(Z_{0}\right)\left(1+\varepsilon R_{0}(z, \xi)\right)$, where $R_{0}$ is a smooth function of the variables $(z, \xi)$, uniformly bounded in $z$ and satisfying

$$
\left|R_{0}(y, \xi)\right| \leq \frac{C \vartheta(y)}{\left(1+|\xi|^{N-2}\right)}
$$

for some positive constant $C$ independent of $\varepsilon$, and some generic function $\vartheta(y)$ defined on $K$, smooth and uniformly bounded as $\varepsilon \rightarrow 0$. Hence, recalling the definition of the function $\mathfrak{b}$ above, a Taylor expansion gives

$$
\mathfrak{b}(t)=-t \int_{K_{\varepsilon} \times \hat{\mathcal{C}}_{\varepsilon}}\left|\partial_{\bar{a}}\left(\frac{\delta}{\mu_{\varepsilon}} \mathcal{T}_{\mu_{\varepsilon}, \bar{\Phi}_{\varepsilon}}\left(Z_{0}\right) \bar{\chi}_{\varepsilon}\right)\right|^{2}(1+O(t)) .
$$


Observe now that

$$
\begin{aligned}
\partial_{\bar{a}}\left(\frac{\delta}{\mu_{\varepsilon}} \mathcal{T}_{\mu_{\varepsilon}, \Phi_{\varepsilon}}\left(Z_{0}\right) \bar{\chi}_{\varepsilon}\right) & =\left(\partial_{\bar{a}} \delta\right) \frac{1}{\mu_{\varepsilon}} \mathcal{T}_{\mu_{\varepsilon}, \Phi_{\varepsilon}}\left(Z_{0}\right) \bar{\chi}_{\varepsilon}+\delta \partial_{\bar{a}}\left(\frac{1}{\mu_{\varepsilon}} \mathcal{T}_{\mu_{\varepsilon}, \Phi_{\varepsilon}}\left(Z_{0}\right) \bar{\chi}_{\varepsilon}\right) \\
& =\varepsilon\left(\partial_{a} \delta\right) \frac{1}{\mu_{\varepsilon}} \mathcal{T}_{\mu_{\varepsilon}, \Phi_{\varepsilon}}\left(Z_{0}\right) \bar{\chi}_{\varepsilon}+\varepsilon \delta\left(\partial_{a} \mu_{\varepsilon}\right) \partial_{\mu_{\varepsilon}}\left(\frac{1}{\bar{\mu}_{\varepsilon}} \mathcal{T}_{\mu_{\varepsilon}, \Phi_{\varepsilon}}\left(Z_{0}\right) \bar{\chi}_{\varepsilon}\right) \\
& +\varepsilon \delta\left(\partial_{a} \Phi_{\varepsilon}\right) \partial_{\bar{\Phi}_{\varepsilon}}\left(\frac{1}{\mu_{\varepsilon}} \mathcal{T}_{\mu_{\varepsilon}, \bar{\Phi}_{\varepsilon}}\left(Z_{0}\right) \bar{\chi}_{\varepsilon}\right)
\end{aligned}
$$

Since $\int\left(\frac{1}{\mu_{\varepsilon}} \mathcal{T}_{\mu_{\varepsilon}, \Phi_{\varepsilon}}\left(Z_{0}\right) \bar{\chi}_{\varepsilon}\right)^{2} d X=A(1+o(\varepsilon))$, we conclude that

$$
\mathfrak{b}(t)=-t \varepsilon^{-k}\left[A_{\varepsilon} \varepsilon^{2} \int_{K}\left|\partial_{a}\left(\delta\left(1+o\left(\varepsilon^{2}\right) \beta_{1}^{\varepsilon}(y)\right)\right)\right|^{2}\right]
$$

where $A_{\varepsilon} \in \mathbb{R}, \lim _{\varepsilon \rightarrow 0} A_{\varepsilon}=A=\int_{\mathbb{R}_{+}^{N}} Z_{0}^{2}$ and $\beta_{1}^{\varepsilon}$ is an explicit smooth function in $K$, which is uniformly bounded as $\varepsilon \rightarrow 0$. Finally we observe that the last term $\mathfrak{c}(t)$ defined above is of lower order, and can be absorbed in the terms described in (9.10) and (9.11).

The expansion (9.1) clearly holds from (9.5)-(9.8)-(9.10) and (9.11).

Step 2: Proof of (9.2). To get the expansion in (9.2) we argue in the same spirit as before. Let $d$ be the vector field along $K$ defined by $d(\varepsilon z)=\left(d^{1}(\varepsilon z), \ldots, d^{N-1}(\varepsilon z)\right)$. For any $t$ small and $t \neq 0$, we have (see (9.4))

$$
\begin{aligned}
& {\left[D F\left(\mathcal{T}_{\mu_{\varepsilon}, \Phi_{\varepsilon}+t d}(\hat{w}) \bar{\chi}_{\varepsilon}\right)-D F\left(\mathcal{T}_{\mu_{\varepsilon}, \Phi_{\varepsilon}}(\hat{w}) \bar{\chi}_{\varepsilon}\right)\right][\varphi]=} \\
& t D^{2} F\left(\mathcal{T}_{\mu_{\varepsilon}, \Phi_{\varepsilon}}(\hat{w}) \bar{\chi}_{\varepsilon}\right)\left[\sum_{l} \frac{d^{l}}{\mu_{\varepsilon}} \mathcal{T}_{\mu_{\varepsilon}, \Phi_{\varepsilon}}\left(Z_{l}\right) \bar{\chi}_{\varepsilon}\right][\varphi](1+O(t))(1+O(\varepsilon))
\end{aligned}
$$

for any function $\varphi \in H_{\varepsilon}^{1}$. In particular, choosing $\varphi=\frac{d^{j}}{\mu_{\varepsilon}} \mathcal{T}_{\mu_{\varepsilon}, \Phi_{\varepsilon}}\left(Z_{j}\right) \bar{\chi}_{\varepsilon}$ we get (using the fact that $\int_{\mathbb{R}_{+}^{N}} Z_{j} Z_{l}=C_{0} \delta_{j l}$ ) that

$$
\begin{aligned}
& {\left[D F\left(\mathcal{T}_{\mu_{\varepsilon}, \Phi_{\varepsilon}+t d}(\hat{w}) \bar{\chi}_{\varepsilon}\right)-D F\left(\mathcal{T}_{\mu_{\varepsilon}, \Phi_{\varepsilon}}(\hat{w}) \bar{\chi}_{\varepsilon}\right)\right]\left[\frac{d^{j}}{\mu_{\varepsilon}} \mathcal{T}_{\mu_{\varepsilon}, \Phi_{\varepsilon}}\left(Z_{j}\right) \bar{\chi}_{\varepsilon}\right]=} \\
& 2 t E\left(\frac{d^{j}}{\mu_{\varepsilon}} \mathcal{T}_{\mu_{\varepsilon}, \Phi_{\varepsilon}}\left(Z_{j}\right) \bar{\chi}_{\varepsilon}\right)(1+O(t))(1+O(\varepsilon)) .
\end{aligned}
$$

On the other hand, as in the previous step, we write

$$
\begin{aligned}
& {\left[D F\left(\mathcal{T}_{\mu_{\varepsilon}, \Phi_{\varepsilon}+t d}(\hat{w}) \bar{\chi}_{\varepsilon}\right)-D F\left(\mathcal{T}_{\mu_{\varepsilon}, \Phi_{\varepsilon}}(\hat{w}) \bar{\chi}_{\varepsilon}\right)\right]\left[\frac{d^{j}}{\bar{\mu}_{\varepsilon}} \mathcal{T}_{\mu_{\varepsilon}, \Phi_{\varepsilon}}\left(Z_{j}\right) \bar{\chi}_{\varepsilon}\right]=} \\
& \mathfrak{a}_{2}(t)-\mathfrak{a}_{2}(0)+\mathfrak{b}_{2}(t)+\mathfrak{c}_{2}(t)
\end{aligned}
$$

where we have set, for $\psi=\frac{d^{j}}{\mu_{\varepsilon}} \mathcal{T}_{\mu_{\varepsilon}, \Phi_{\varepsilon}}\left(Z_{j}\right) \bar{\chi}_{\varepsilon}$,

$$
\begin{aligned}
\mathfrak{a}_{2}(t) & =\int\left(\nabla_{X} \mathcal{T}_{\mu_{\varepsilon}, \Phi_{\varepsilon}+t d}(\hat{w}) \bar{\chi}_{\varepsilon}\right) \nabla_{X} \psi+\varepsilon \mathcal{T}_{\mu_{\varepsilon}, \Phi_{\varepsilon}+t d}(\hat{w}) \bar{\chi}_{\varepsilon} \psi-\left(\mathcal{T}_{\mu_{\varepsilon}, \Phi_{\varepsilon}+t d}\left(\hat{w} \bar{\chi}_{\varepsilon}\right)\right)^{p} \psi \\
& +\int \Xi_{i j}(\varepsilon z, X) \partial_{i}\left(\mathcal{T}_{\mu_{\varepsilon}, \Phi_{\varepsilon}+t d}(\hat{w}) \bar{\chi}_{\varepsilon}\right) \partial_{j} \psi
\end{aligned}
$$


$\mathfrak{b}_{2}(t)=\int \partial_{\bar{a}}\left(\mathcal{T}_{\bar{\mu}_{\varepsilon}, \Phi_{\varepsilon}+t d}(\hat{w}) \bar{\chi}_{\varepsilon}\right) \partial_{\bar{a}}\left(\mathcal{T}_{\mu_{\varepsilon}, \Phi_{\varepsilon}+t d}(\hat{w}) \bar{\chi}_{\varepsilon}\right)-\int \partial_{\bar{a}}\left(\mathcal{T}_{\mu_{\varepsilon}, \Phi_{\varepsilon}}(\hat{w}) \bar{\chi}_{\varepsilon}\right) \partial_{\bar{a}}\left(\mathcal{T}_{\mu_{\varepsilon}, \Phi_{\varepsilon}}(\hat{w}) \bar{\chi}_{\varepsilon}\right)$

and

$$
\mathfrak{c}_{2}(t)=\int B\left(\mathcal{T}_{\bar{\mu}_{\varepsilon}, \bar{\Phi}_{\varepsilon}+t d}(\hat{w}) \bar{\chi}_{\varepsilon}, \psi\right)-\int B\left(\mathcal{T}_{\bar{\mu}_{\varepsilon}, \Phi_{\varepsilon}}(\hat{w}) \bar{\chi}_{\varepsilon}, \psi\right)
$$

We now compute $\mathfrak{a}_{2}(t)$ with $\psi=\frac{\delta}{\bar{\mu}_{\varepsilon}} \mathcal{T}_{\mu_{\varepsilon}, \Phi_{\varepsilon}}\left(Z_{j}\right)$. Defining the tensor $\mathcal{R}_{m l}$ by

$$
\mathcal{R}_{m l}=\left(\left(\tilde{g}^{\varepsilon}\right)^{a b} R_{m a b l}-\Gamma_{a}^{c}\left(E_{m}\right) \Gamma_{c}^{a}\left(E_{l}\right)\right)
$$

performing the change of variables $\bar{X}=\left(\mu_{\varepsilon}+t \delta\right) \bar{\xi}+\Phi_{\varepsilon}, X_{N}=\left(\bar{\mu}_{\varepsilon}+t \delta\right) \xi_{N}$ in the integral $\mathfrak{a}_{2}(t)$, using (3.9), (5.16) and recalling the definition of the cut-off function $\bar{\chi}_{\varepsilon}$, we get

$$
\begin{aligned}
& \mathfrak{a}_{2}(t)=\left\{\int \frac{d^{j}}{\mu_{\varepsilon}}\left[\nabla \hat{w} \nabla Z_{j}+\varepsilon \mu_{\varepsilon}^{2} \hat{w} Z_{j}-p \hat{w}^{p} Z_{j}\right] \times\left[1-\varepsilon \mu_{\varepsilon} \xi_{N} H_{\alpha \alpha}\right.\right. \\
& \left.+\varepsilon^{2}\left(\frac{R_{m i i l}}{6}+\frac{\mathcal{R}_{m l}}{2}\right)\left(\varepsilon \mu_{\varepsilon} \xi_{m}+\Phi_{\varepsilon m}+t d^{m}\right)\left(\varepsilon \bar{\mu}_{\varepsilon} \xi_{l}+\Phi_{\varepsilon l}+t d^{l}\right)+O\left(\varepsilon^{3}|\xi|^{3}\right)\right] \\
& +\int \frac{d^{j}}{\mu_{\varepsilon}}\left[2 \varepsilon \xi_{N} H_{i r}-\frac{\varepsilon^{2}}{3} R_{i m l r}\left(\mu_{\varepsilon} \xi_{m}+\Phi_{\varepsilon m}+t d^{m}\right)\left(\mu_{\varepsilon} \xi_{l}+\Phi_{\varepsilon l}+t d^{l}\right)\right. \\
& \left.\left.\quad+O\left(\varepsilon^{3}|\xi|^{3}\right)\right] \partial_{i} \hat{w} \partial_{r} Z_{j}\right\} \times \\
& \times(1+O(t))\left(1+O(\varepsilon)+O\left(\varepsilon^{\gamma(N-3)}\right)\right) .
\end{aligned}
$$

Thus we immediately get (using the fact that $\gamma(N-3)>1$ )

$$
\begin{aligned}
& t^{-1}\left[\mathfrak{a}_{2}(t)-\mathfrak{a}_{2}(0)\right]=\varepsilon^{2}\left\{\int \frac{d^{j}}{\mu_{\varepsilon}}\left[\nabla \hat{w} \nabla Z_{j}+\varepsilon \bar{\mu}_{\varepsilon}^{2} \hat{w} Z_{j}-p \hat{w}^{p} Z_{j}\right] \times\right. \\
& \times\left(\frac{R_{m i j l}}{6}+\frac{\mathcal{R}_{l m}}{2}\right)\left[\left(\bar{\mu}_{\varepsilon} \xi_{m}+\Phi_{\varepsilon m}\right) d^{l}+\left(\mu_{\varepsilon} \xi_{l}+\bar{\Phi}_{\varepsilon l}\right) d^{m}\right] \\
& \left.-\int \frac{d^{j}}{\mu_{\varepsilon}} \frac{R_{i l m r}}{3}\left[\left(\bar{\mu}_{\varepsilon} \xi_{m}+\Phi_{\varepsilon m}\right) d^{l}+\left(\mu_{\varepsilon} \xi_{l}+\Phi_{\varepsilon l}\right) d^{m}\right] \partial_{i} \hat{w} \partial_{r} Z_{j}\right\}(1+O(\varepsilon))(1+O(t)) .
\end{aligned}
$$

Integration by parts in the $\xi$ variables and using the fact that $\hat{\mathcal{C}}_{\varepsilon} \rightarrow \mathbb{R}_{+}^{N}$ as $\varepsilon \rightarrow 0$, we get

$$
\begin{aligned}
& t^{-1}\left[\mathfrak{a}_{2}(t)-\mathfrak{a}_{2}(0)\right]=\varepsilon^{2}\left\{\int \frac{d^{j}}{\mu_{\varepsilon}}\left[-\Delta \hat{w}+\varepsilon \mu_{\varepsilon} \hat{w}-p \hat{w}\right] Z_{j} \times\right. \\
& \times\left(\frac{R_{m i j l}}{6}+\frac{\mathcal{R}_{l m}}{2}\right)\left[\left(\mu_{\varepsilon} \xi_{m}+\Phi_{\varepsilon m}\right) d^{l}+\left(\mu_{\varepsilon} \xi_{l}+\Phi_{\varepsilon l}\right) d^{m}\right] \\
& \left.-\int\left(\frac{R_{m i j l}}{6}+\frac{\mathcal{R}_{l m}}{2}\right) d^{j}\left[\partial_{l} \hat{w} d^{m}+\partial_{m} \hat{w} d^{l}\right] Z_{j}+\int d^{j}\left[\frac{R_{i l r r}}{3} d^{l}+\frac{R_{i r m r}}{3} d^{m}\right] Z_{i} Z_{j}\right\} \times \\
& \times(1+O(\varepsilon))(1+O(t)) .
\end{aligned}
$$


Now using the fact that $\left\|-\Delta \hat{w}+\varepsilon \mu_{\varepsilon}^{2} \hat{w}-\hat{w}^{p}\right\|_{\varepsilon, N-2} \leq C \varepsilon^{3}$ and that $R_{i l r r}=0$, we deduce that

$$
\begin{aligned}
t^{-1}\left[\mathfrak{a}_{2}(t)-\mathfrak{a}_{2}(0)\right] & =\varepsilon^{2}\left\{-C \int_{K_{e}}\left(\frac{R_{m i i j}}{3}+\frac{\mathcal{R}_{m j}}{2}\right) d^{j} d^{m}+C \int_{K_{\varepsilon}} \frac{R_{\text {jrrm }}}{3} d^{m} d^{j}\right\} \times \\
& \times(1+o(\varepsilon))(1+O(t)) \\
& =\varepsilon^{-k} \varepsilon^{2}\left[-C \int \frac{\mathcal{R}_{m j}}{2} d^{j} d^{m}+O(\varepsilon) Q(d)\right](1+O(t))
\end{aligned}
$$

where here we have set

$$
C=\int_{\mathbb{R}_{+}^{N}} Z_{1}^{2} \quad \text { and } \quad Q(d):=\int_{K} \pi(y) d^{i} d^{j}
$$

for some smooth and uniformly bounded (as $\varepsilon \rightarrow 0$ ) function $\pi(y)$. To estimate the term $\mathfrak{b}_{2}$ above we argue as in (9.11), we get that

$$
t^{-1} \mathfrak{b}_{2}(t)=-\varepsilon^{-k}\left[\varepsilon^{2} C_{\varepsilon} \int_{K}\left|\partial_{a}\left(d^{j}\left(1+\beta_{2}^{\varepsilon}(y) o\left(\varepsilon^{2}\right)\right)\right)\right|^{2}\right](1+O(t)) .
$$

Finally we observe that the last term $\mathfrak{c}_{2}(t)$ is of lower order, and can be absorbed in the terms described in (9.14) and (9.15). We get the expansion (9.2) from (9.12)-(9.13)-(9.14) and (9.15).

Step 3: Proof of (9.3). To get the expansion in (9.3), we compute

$$
E\left(\frac{e}{\mu_{\varepsilon}} \mathcal{T}_{\mu_{\varepsilon}, \Phi_{\varepsilon}}(Z)\right)=I+I I+I I I
$$

where

$$
\begin{aligned}
I & =\int_{K_{\varepsilon} \times \hat{\mathcal{C}}_{\varepsilon}} \frac{\delta^{2}}{\mu_{\varepsilon}^{2}}\left(\frac{1}{2}\left(\left|\nabla_{X} \mathcal{T}_{\mu_{\varepsilon}, \Phi_{\varepsilon}}(Z)\right|^{2}+\varepsilon \mathcal{T}_{\mu_{\varepsilon}, \Phi_{\varepsilon}}(Z)^{2}-p V_{\varepsilon}^{p-1} \mathcal{T}_{\mu_{\varepsilon}, \Phi_{\varepsilon}}(Z)^{2}\right)\right) \sqrt{\operatorname{det} g^{\varepsilon}} d z d X \\
& +\int_{K_{\varepsilon} \times \hat{\mathcal{C}}_{\varepsilon}} \frac{\delta^{2}}{\mu_{\varepsilon}^{2}} \frac{1}{2} \Xi_{i j}(\varepsilon z, X) \partial_{i} \mathcal{T}_{\bar{\mu}_{\varepsilon}, \Phi_{\varepsilon}}(Z) \partial_{j} \mathcal{T}_{\mu_{\varepsilon}, \Phi_{\varepsilon}}(Z) \sqrt{\operatorname{det} g^{\varepsilon}} d z d X, \\
I I & =\frac{1}{2} \int_{K_{\varepsilon} \times \hat{\mathcal{C}}_{\varepsilon}} \partial_{\bar{a}}\left(\frac{e}{\mu_{\varepsilon}} \mathcal{T}_{\mu_{\varepsilon}, \Phi_{\varepsilon}}(Z)\right) \partial_{\bar{a}}\left(\frac{e}{\mu_{\varepsilon}} \mathcal{T}_{\mu_{\varepsilon}, \Phi_{\varepsilon}}(Z)\right) \sqrt{\operatorname{det} g^{\varepsilon}} d z d X
\end{aligned}
$$

and

$I I I=\int_{K_{\varepsilon} \times \hat{\mathcal{C}}_{\varepsilon}} B\left(\frac{e}{\mu_{\varepsilon}} \mathcal{T}_{\mu_{\varepsilon}, \Phi_{\varepsilon}}(Z), \frac{e}{\mu_{\varepsilon}} \mathcal{T}_{\mu_{\varepsilon}, \Phi_{\varepsilon}}(Z)\right) \sqrt{\operatorname{det}\left(g^{\varepsilon}\right)} d z d X$.

Using the change of variables $\bar{X}=\mu_{\varepsilon} \bar{\xi}+\bar{\Phi}_{\varepsilon}, X_{N}=\mu_{\varepsilon} \xi_{N}$ in $I$, we can write

$$
I=\int \frac{1}{2} \frac{\delta^{2}}{\mu_{\varepsilon}^{2}}\left[|\nabla Z|^{2}-p \hat{w}^{p-1} Z^{2}+\varepsilon \mu_{\varepsilon}^{2} Z^{2}\right]\left(1+\varepsilon O\left(e^{-|\xi|}\right)\right) .
$$


Then, recalling the definition of $\lambda_{0}$ in (5.20), we get

$$
I=\varepsilon^{-k}\left[-\frac{\lambda_{0}}{2} D \int_{K} e^{2}+\varepsilon Q(e)\right]
$$

where we have set

$$
D=\int_{\mathbb{R}_{+}^{N}} Z^{2}(\xi) d \xi \quad \text { and } \quad Q(e):=\int_{K} \tau(y) e^{2} d y
$$

for some smooth and uniformly bounded, as $\varepsilon \rightarrow 0$, function $\tau$. On the other hand, using a direct computation and arguing as in (9.11), we get

$$
I I=\frac{D_{\varepsilon}}{2} \int_{K_{\varepsilon}}\left|\partial_{\bar{a}} e+e^{-\lambda_{0} \varepsilon^{-\gamma}} \beta_{3}^{\varepsilon}(\varepsilon z) e\right|^{2}=\varepsilon^{-k}\left[\frac{D_{\varepsilon}}{2} \varepsilon^{2} \int_{K}\left|\partial_{a}\left(e\left(1+e^{-\lambda^{\prime} \varepsilon^{-\gamma}} \beta_{3}^{\varepsilon}(y)\right)\right)\right|^{2}\right]
$$

where $\beta_{3}^{\varepsilon}$ is an explicit smooth function on $K$, which is uniformly bounded as $\varepsilon \rightarrow 0$, while $\lambda^{\prime}$ is a positive real number. Finally we observe that the last term $I I I$ is of lower order, and can be absorbed in the terms described in (9.17) and (9.18). This concludes the proof of (9.3).

We have now the elements to prove Theorem 2.

Proof of Theorem 2. Given the result in Lemma 9.1, we can write

$$
\begin{aligned}
\mathcal{M}\left(\phi^{\perp}, \delta, d, e\right) & =E(\phi)-E\left(\phi^{\perp}\right)-E\left(\frac{\delta}{\mu_{\varepsilon}} \mathcal{T}_{\mu_{\varepsilon}, \Phi_{\varepsilon}}\left(Z_{0}\right) \bar{\chi}_{\varepsilon}\right)-\sum_{j=1}^{N-1} E\left(\frac{d_{j}}{\mu_{\varepsilon}} \mathcal{T}_{\mu_{\varepsilon}, \Phi_{\varepsilon}}\left(Z_{j}\right) \bar{\chi}_{\varepsilon}\right) \\
& -E\left(\frac{e}{\mu_{\varepsilon}} \mathcal{T}_{\mu_{\varepsilon}, \Phi_{\varepsilon}}(Z) \bar{\chi}_{\varepsilon}\right) .
\end{aligned}
$$

Thus it is clear that the term $\mathcal{M}$ recollects all the mixed terms in the expansion of $E(\phi)$. Indeed, if we define

$$
\begin{aligned}
m(f, g) & =\int_{K_{\varepsilon} \times \hat{\mathcal{C}}_{\varepsilon}}\left(\nabla_{X} f \nabla_{X} g+\varepsilon f g-p V_{\varepsilon}^{p-1} f g\right) \sqrt{\operatorname{det}\left(g^{\varepsilon}\right)} d z d X \\
& +\int_{K_{\varepsilon} \times \hat{\mathcal{C}}_{\varepsilon}} \Xi_{i j}(\varepsilon z, X) \partial_{i} f \partial_{j} g \sqrt{\operatorname{det}\left(g^{\varepsilon}\right)} d z d X \\
& +\int_{K_{\varepsilon} \times \hat{\mathcal{C}}_{\varepsilon}} \partial_{\bar{a}} f \partial_{\bar{a}} g \sqrt{\operatorname{det}\left(g^{\varepsilon}\right)} d z d X+\int_{K_{\varepsilon} \times \hat{\mathcal{C}}_{\varepsilon}} B(f, g) \sqrt{\operatorname{det}\left(g^{\varepsilon}\right)} d z d X
\end{aligned}
$$


for $f$ and $g$ in $H_{\varepsilon}^{1}$, then

$$
\begin{aligned}
\mathcal{M}\left(\phi^{\perp}, \delta, d, e\right) & =m\left(\phi^{\perp}, \frac{\delta}{\mu_{\varepsilon}} \mathcal{T}_{\mu_{\varepsilon}, \Phi_{\varepsilon}}\left(Z_{0}\right) \bar{\chi}_{\varepsilon}\right)+\sum_{j} m\left(\phi^{\perp}, \frac{d_{j}}{\mu_{\varepsilon}} \mathcal{T}_{\mu_{\varepsilon}, \Phi_{\varepsilon}}\left(Z_{j}\right) \bar{\chi}_{\varepsilon}\right) \\
& +m\left(\phi^{\perp}, \frac{e}{\mu_{\varepsilon}} \mathcal{T}_{\mu_{\varepsilon}, \Phi_{\varepsilon}}(Z) \bar{\chi}_{\varepsilon}\right)+\sum_{j} m\left(\frac{\delta}{\bar{\mu}_{\varepsilon}} \mathcal{T}_{\mu_{\varepsilon}, \Phi_{\varepsilon}}\left(Z_{0}\right) \bar{\chi}_{\varepsilon}, \frac{d^{j}}{\mu_{\varepsilon}} \mathcal{T}_{\mu_{\varepsilon}, \bar{\Phi}_{\varepsilon}}\left(Z_{j}\right) \bar{\chi}_{\varepsilon}\right) \\
& +\sum_{i \neq j} m\left(\frac{d^{j}}{\mu_{\varepsilon}} \mathcal{T}_{\bar{\mu}_{\varepsilon}, \Phi_{\varepsilon}}\left(Z_{j}\right) \bar{\chi}_{\varepsilon}, \frac{d_{i}}{\bar{\mu}_{\varepsilon}} \mathcal{T}_{\mu_{\varepsilon}, \Phi_{\varepsilon}}\left(Z_{i}\right) \bar{\chi}_{\varepsilon}\right) \\
& +m\left(\frac{\delta}{\mu_{\varepsilon}} \mathcal{T}_{\mu_{\varepsilon}, \bar{\Phi}_{\varepsilon}}\left(Z_{0}\right) \bar{\chi}_{\varepsilon}, \frac{e}{\mu_{\varepsilon}} \mathcal{T}_{\mu_{\varepsilon}, \Phi_{\varepsilon}}(Z) \bar{\chi}_{\varepsilon}\right) \\
& +\sum_{j} m\left(\frac{d^{j}}{\mu_{\varepsilon}} \mathcal{T}_{\mu_{\varepsilon}, \Phi_{\varepsilon}}\left(Z_{j}\right) \bar{\chi}_{\varepsilon}, \frac{e}{\mu_{\varepsilon}} \mathcal{T}_{\mu_{\varepsilon}, \Phi_{\varepsilon}}(Z) \bar{\chi}_{\varepsilon}\right) .
\end{aligned}
$$

One can see clearly that $\mathcal{M}$ is homogeneous of degree 2 and that its first derivatives with respect to its variables is a linear operator in $\left(\phi^{\perp}, \delta, d, e\right)$. We will then show the validity of estimate (5.32). In a very similar way one shows the validity of (5.31). To prove (5.32), we should treat each one of the above terms. Since the computations are very similar, we will limit ourselves to treat the term

$$
m:=m\left(\frac{\delta}{\mu_{\varepsilon}} \mathcal{T}_{\mu_{\varepsilon}, \Phi_{\varepsilon}}\left(Z_{0}\right) \bar{\chi}_{\varepsilon}, \frac{d^{j}}{\mu_{\varepsilon}} \mathcal{T}_{\mu_{\varepsilon}, \Phi_{\varepsilon}}\left(Z_{j}\right) \bar{\chi}_{\varepsilon}\right)
$$

This term can be written as

$$
m=\sum_{i=1}^{5} m_{i}
$$

where

$$
\begin{gathered}
m_{1}=\int_{K_{\varepsilon} \times \hat{\mathcal{C}}_{\varepsilon}}\left(\nabla_{X} f \nabla_{X} g-p V_{\varepsilon}^{p-1} f g\right) \sqrt{\operatorname{det}\left(g^{\varepsilon}\right)} d z d X \\
m_{2}=\int_{K_{\varepsilon} \times \hat{\mathcal{C}}_{\varepsilon}} \varepsilon f g \sqrt{\operatorname{det}\left(g^{\varepsilon}\right)} d z d X, \quad m_{3}=\int_{K_{\varepsilon} \times \hat{\mathcal{C}}_{\varepsilon}} \Xi_{i j}(\varepsilon z, X) \partial_{i} f \partial_{j} g \sqrt{\operatorname{det}\left(g^{\varepsilon}\right)} d z d X \\
m_{4}=\int_{K_{\varepsilon} \times \hat{\mathcal{C}}_{\varepsilon}} \partial_{\bar{a}} f \partial_{\bar{a}} g \sqrt{\operatorname{det}\left(g^{\varepsilon}\right)} d z d X \quad \text { and } m_{5}=\int_{K_{\varepsilon} \times \hat{\mathcal{C}}_{\varepsilon}} B(f, g) \sqrt{\operatorname{det}\left(g^{\varepsilon}\right)} d z d X
\end{gathered}
$$

with $f=\frac{\delta}{\mu_{\varepsilon}} \mathcal{T}_{\mu_{\varepsilon}, \bar{\Phi}_{\varepsilon}}\left(Z_{0}\right) \bar{\chi}_{\varepsilon}$ and $g=\frac{d^{j}}{\mu_{\varepsilon}} \mathcal{T}_{\mu_{\varepsilon}, \Phi_{\varepsilon}}\left(Z_{j}\right) \bar{\chi}_{\varepsilon}$. Using the fact that the function $Z_{0}$ solves

$$
\Delta Z_{0}+p w_{0}^{p-1} Z_{0}=0 \text { in } \quad \mathbb{R}^{N},
$$

with $\int_{\mathbb{R}_{+}^{N}} \partial_{\xi_{N}} Z_{0} Z_{j}=0$ and integrating by parts in the $X$ variable (recalling the expansion of $\sqrt{\operatorname{det} g^{\varepsilon}}$ ), one gets

$$
\begin{aligned}
m_{1} & =\left\{\int \frac{\delta d^{j}}{\mu_{\varepsilon}^{2}}\left[-\Delta \mathcal{T}_{\mu_{\varepsilon}, \Phi_{\varepsilon}}\left(Z_{0}\right)-p V_{\varepsilon}^{p-1} \mathcal{T}_{\mu_{\varepsilon}, \Phi_{\varepsilon}}\left(Z_{0}\right)\right] \bar{\chi}_{\varepsilon}^{2} \mathcal{T}_{\mu_{\varepsilon}, \Phi_{\varepsilon}}\left(Z_{j}\right) \sqrt{\operatorname{det} g^{\varepsilon}}\right. \\
& \left.+\int \frac{\delta d^{j}}{\mu_{\varepsilon}^{2}} \partial_{\xi_{N}}\left(\mathcal{T}_{\mu_{\varepsilon}, \Phi_{\varepsilon}}\left(Z_{0}\right) \bar{\chi}_{\varepsilon}\right) \mathcal{T}_{\mu_{\varepsilon}, \Phi_{\varepsilon}}\left(Z_{j}\right) \frac{1}{\mu_{\varepsilon}}\left(\varepsilon \operatorname{tr}(H)+O\left(\varepsilon^{2}\right)\right) \bar{\chi}_{\varepsilon}\right\}(1+o(1))
\end{aligned}
$$


where $o(1) \rightarrow 0$ as $\varepsilon \rightarrow 0$. Thus, a Hölder inequality yields

$$
\left|m_{1}\right| \leq C \varepsilon^{-k} \varepsilon^{\gamma(N-2)}\|\delta\|_{\mathcal{L}^{2}(K)}\left\|d^{j}\right\|_{\mathcal{L}^{2}(K)}
$$

On the other hand, using the orthogonality condition $\int_{\mathbb{R}_{+}^{N}} Z_{0} Z_{j}=0$, we get

$$
\left|m_{2}\right| \leq C \varepsilon \varepsilon^{-k}\left(\int_{|\xi|>\varepsilon^{-\gamma}} Z_{0} Z_{j}\right)\|\delta\|_{\mathcal{L}^{2}(K)}\left\|d^{j}\right\|_{\mathcal{L}^{2}(K)} \leq C \varepsilon^{-k} \varepsilon^{1+\gamma(N-3)}\|\delta\|_{\mathcal{L}^{2}(K)}\left\|d^{j}\right\|_{\mathcal{L}^{2}(K)}
$$

Now, since $\int_{\mathbb{R}_{+}^{N}} \xi_{N} \partial_{i} Z_{0} \partial_{l} Z_{j}=0$, for any $i, j, l=1, \ldots, N-1$, one gets

$$
\begin{aligned}
\left|m_{3}\right| & \leq C \varepsilon \varepsilon^{-k}\left(\int_{|\xi|>\varepsilon^{-\gamma}} \xi_{N} \partial_{i} Z_{0} \partial_{l} Z_{j}\right)\|\delta\|_{\mathcal{L}^{2}(K)}\left\|d^{j}\right\|_{\mathcal{L}^{2}(K)} \\
& \leq C \varepsilon^{-k} \varepsilon^{1+\gamma(N-2)}\|\delta\|_{\mathcal{L}^{2}(K)}\left\|d^{j}\right\|_{\mathcal{L}^{2}(K)}
\end{aligned}
$$

A direct computation on the term $m_{4}$ gives

$$
\begin{aligned}
\left|m_{4}\right| & \leq C \varepsilon^{-k}\left\{\varepsilon^{2}\left(\int_{|\xi|>\varepsilon^{-\gamma}} Z_{0} Z_{j}\right)\left\|\partial_{a} \delta\right\|_{\mathcal{L}^{2}(K)}\left\|\partial_{a} d^{j}\right\|_{\mathcal{L}^{2}(K)}\right. \\
& +\varepsilon\left(\int_{|\xi|>\varepsilon^{-\gamma}} Z_{0} Z_{j}\right)\left(\|\delta\|_{\mathcal{L}^{2}(K)}\left\|\partial_{a} d^{j}\right\|_{\mathcal{L}^{2}(K)}+\left\|\partial_{a} \delta\right\|_{\mathcal{L}^{2}(K)}\left\|d^{j}\right\|_{\mathcal{L}^{2}(K)}\right) \\
& \left.+\left(\int_{|\xi|>\varepsilon^{-\gamma}} Z_{0} Z_{j}\right)\|\delta\|_{\mathcal{L}^{2}(K)}\left\|d^{j}\right\|_{\mathcal{L}^{2}(K)}\right\} \\
& \leq C \varepsilon^{-k} \varepsilon^{\gamma(N-3)}\left[\|\delta\|_{\mathcal{H}^{1}(K)}^{2}+\left\|d^{j}\right\|_{\mathcal{H}^{1}(K)}^{2}\right]
\end{aligned}
$$

Since $\left|m_{5}\right| \leq C \sum_{j=1}^{4}\left|m_{j}\right|$ we conclude that

$$
|m| \leq C \varepsilon^{-k} \varepsilon^{\gamma(N-3)}\left[\|\delta\|_{\mathcal{H}^{1}(K)}^{2}+\left\|d^{j}\right\|_{\mathcal{H}^{1}(K)}^{2}\right] .
$$

Each one of the terms appearing in (9.19) can be estimated to finally get the validity of (5.32). This conclude the proof of Theorem 2.

Acknowledgments. This work has been supported by grants Fondecyt 1110181, 1100164 and 1120151, and Fondo Basal CMM. We would like to thank the anonymous referees for a careful reading of the paper, which meant an important improvement of the original version of the paper. 


\section{References}

[1] Adimurthi, Mancini, G. The Neumann problem for elliptic equations with critical nonlinearity, A tribute in honour of G. Prodi. Scuola Norm. Sup. Pisa 9-25 (1991).

[2] Adimurthi, Mancini, G. Geometry and topology of the boundary in the critical Neumann problem. J. Reine Angew. Math. 456, 1-18 (1994).

[3] Adimurthi, Mancini G., Yadava S.L., The role of the mean curvature in semilinear Neumann problem involving critical exponent, Comm. Partial Differential Equations 20 (3-4) (1995) 591-631.

[4] Adimurthi, Pacella, F., Yadava, S.L. Interaction between the geometry of the boundary and positive solutions of a semilinear Neumann problem with critical nonlinearity. J. Funct. Anal. 113, 318-350 (1993).

[5] Adimurthi, Pacella F., Yadava S. L., Characterization of concentration points and L1- estimates for solutions of a semilinear Neumann problem involving the critical Sobolev exponent, Diff. Integ. Equ. 8 (1995), 42-68.

[6] Ao W., Musso M., Wei J. On Spikes Concentrating on Line-Segments to a Semilinear Neumann Problem. J. Differential Equations 251 (2011), no. 4-5, 881-901.

[7] Ao W., Musso M., Wei J. Triple Junction Solutions for a Singularly Perturbed Neumann Problem. SIAM J. Math. Anal. 43 (2011), no. 6, 2519-2541.

[8] Byeon, J. Singularly perturbed nonlinear Neumann problems with a general nonlinearity. J. Differential Equations 244 (2008), no. 10, 2473-2497.

[9] Caffarelli, L., Gidas, B., Spruck, J. Asymptotic symmetry and local behavior of semilinear elliptic equations with critical Sobolev growth. Comm. Pure Appl. Math. 42, 271-297 (1989).

[10] Cao, D., Kupper, T. On the existence of multipeaked solutions to a semilinear Neumann problem. Duke Math. J. 97 (1999), no. 2, 261-300.

[11] I. Chavel, Riemannian Geometry, a Modern Introduction, Cambridge Tracts in Math., vol. 108, Cambridge Univ. Press, Cambridge, 1993.

[12] Dancer, E. N., Yan, S. Multipeak solutions for a singularly perturbed Neumann problem. Pacific J. Math. 189, 241-262 (1999). 
[13] del Pino, M., Felmer, P. Spike-layered solutions of singularly perturbed elliptic problems in a degenerate setting. Indiana Univ. Math. J. 48 no. 3, 883-898 (1999).

[14] del Pino, M., Felmer, P., Wei, J. On the role of mean curvature in some singularly perturbed Neumann problems. SIAM J. Math. Anal. 31, 63-79 (2000).

[15] del Pino, M., Kowalczyk, M., Wei J., Yang J., Interface foliations near minimal submanifolds in Riemannian manifolds with positive Ricci curvature. Geom. Functional Analysis No. 20 (2010) 918-957.

[16] del Pino, M., Musso, M., Pistoia, A. Supercritical boundary bubbling in a semilinear Neumann problem. Ann. Inst. H. Poincare Anal. Non-Linearie 22 (1), 45-82 (2005).

[17] del Pino, M., Musso M., Pacard F., Bubbling along geodesics near the second critical exponent. J. Eur. Math. Soc. (JEMS) 12 (2010), no. 6, 1553-1605.

[18] Ghoussoub, N., Gui, C. Multi-peak solutions for a semilinear Neumann problem involving the critical Sobolev exponent. Math. Z. 229, 443-474 (1998).

[19] Ghoussoub, N., Gui, C., Zhu, M. On a singularly perturbed Neumann problem with the critical exponent. Comm. Partial Differential Equations 26, 1929-1946 (2001).

[20] Gierer, A., Meinhardt, H. A theory of biological pattern formation. Kybernetik (Berlin) 12, 30-39 (1972).

[21] Grossi, M., Pistoia, A., Wei, J. Existence of multipeak solutions for a semilinear elliptic problem via nonsmooth critical point theory. Calc. Var. Partial Differential Equations 11, 143-175 (2000).

[22] Gui, C. Multi-peak solutions for a semilinear Neumann problem. Duke Math. J. 84, 739-769 (1996).

[23] Gui, C., Lin, C.-S. Estimates for boundary-bubbling solutions to an elliptic Neumann problem. J. Reine Angew. Math. 546, 201-235 (2002).

[24] Gui, C., Wei, J. Multiple interior peak solutions for some singularly perturbed Neumann problems. J. Differential Equations 158, 1-27 (1999).

[25] Li, Y.Y. On a singularly perturbed equation with Neumann boundary condition. Comm. Partial Differential Equations 23, 487-545 (1998). 
[26] Lin, C.-S. Locating the peaks of solutions via the maximum principle, I. Neumann problem. Comm. Pure Appl. Math. 54, 1065-1095 (2001).

[27] Lin, C.-S., Ni,W.-M., Takagi, I.Large amplitude stationary solutions to a chemotaxis system. J. Differential Equations 72, 1-27 (1988).

[28] Lin, C.-S. Wang, L., Wei, J. Bubble accumulations in an elliptic Neumann problem with critical Sobolev exponent. Calc. Var. Partial Differential Equations 30 (2007), no. 2, 153-182.

[29] Lin, F.-H. , Ni, W.-M., Wei, J. On the number of interior peak solutions for a singularly perturbed Neumann problem. Comm. Pure Appl. Math. 60 (2007), no. 2, 252-281.

[30] Maier-Paape, S., Schmitt, K.,Wang, Z.Q. On Neumann problems for semilinear elliptic equations with critical nonlinearity existence and symmetry of multipeaked solutions. Comm. Partial Differential Equations 22, 1493-1527 (1997).

[31] Mahmoudi F., Malchiodi A., Concentration on minimal submanifolds for a singularly perturbed Neumann problem. Adv. Math. 209 (2007), no. 2, 460-525.

[32] Mahmoudi, F., Mazzeo, R., Pacard, F. Constant mean curvature hypersurfaces condensing along a submanifold. Geom. funct. anal. Vol. 16 (2006) 924-958.

[33] Malchiodi A., Montenegro M., Boundary concentration phenomena for a singularly perturbed elliptic problem, Comm. Pure Appl. Math, 15 (2002), 1507-1568.

[34] Malchiodi A., Montenegro M., Multidimensional Boundary-layers for a singularly perturbed Neumann problem, Duke Math. J. 124:1 (2004), 105-143.

[35] Malchiodi A., Concentration at curves for a singularly perturbed Neumann problem in three-dimensional domains. Geom. Funct. Anal. 15 (2005), no. 6, $1162-$ 1222.

[36] Ni, W.-M. Diffusion, cross-diffusion, and their spike-layer steady states. Notices Amer. Math. Soc. 45 (1998), no. 1, 9-18.

[37] Ni, W.-M. Qualitative properties of solutions to elliptic problems. In Stationary Partial Differential Equations. Handbook Differential Equations, vol. I, pp. 157233. North-Holland, Amsterdam (2004).

[38] Ni, W.-M., Pan, X.-B., Takagi, I. Singular behavior of least-energy solutions of a semi-linear Neumann problem involving critical Sobolev exponents. Duke Math. J. 67, 1-20 (1992). 
[39] Ni, W.-M., Takagi, I. On the shape of least-energy solutions to a semi-linear problem Neumann problem. Comm. Pure Appl. Math. 44, 819-851 (1991).

[40] Ni, W.-M., Takagi, I. Locating the peaks of least-energy solutions to a semi-linear Neumann problem. Duke Math. J. 70, 247-281 (1993).

[41] S. Pohozaev, Eigenfunctions of the equation $\Delta u+\lambda f(u)=0$, Soviet. Math. Dokl. 6, (1965), 1408-1411.

[42] Rey, O. Boundary effect for an elliptic Neumann problem with critical nonlinearity, Comm. Part. Diff. Equ. 22 (1997), 1055-1139.

[43] Rey, O. An elliptic Neumann problem with critical nonlinearity in three dimensional domains. Comm. Contemp. Math. 1, 405-449 (1999).

[44] Rey, O. The question of interior blow-up points for an elliptic Neumann problem: the critical case. J. Math. Pures Appl. 81, 655-696 (2002).

[45] Rey, O. The role of the Green's function in a nonlinear elliptic equation involving the critical Sobolev exponent. J. Funct. Anal. 89 (1990), no. 1, 1-52.

[46] Rey, O., Wei, J. Arbitrary number of positive solutions for an elliptic problem with critical nonlinearity. J. Eur. Math. Soc. (JEMS) 7, 449-476 (2005).

[47] R. Schoen and S.T. Yau, Lectures on Differential Geometry, International Press (1994).

[48] Wang, X.-J. Neumann problem of semilinear elliptic equations involving critical Sobolev exponents. J. Differential Equations 93, 283-310 (1991).

[49] Wang, Z.Q. High energy and multi-peaked solutions for a nonlinear Neumann problem with critical exponent. Proc. Roy. Soc. Edimburgh 125A, 1003-1029 (1995).

[50] Wang, L., Wei, J., Yan, S. A Neumann problem with critical exponent in nonconvex domains and Lin-Ni's conjecture. Trans. Amer. Math. Soc. 362 (2010), no. 9, 4581-4615.

[51] Wei J., On the boundary spike layer solutions of a singularly perturbed semilinear Neumann problem, J. Differential Equations 134 (1997) 104-133.

[52] Wei, J., Xu, X. Uniqueness and a priori estimates for some nonlinear elliptic Neumann equations in R3. Pacific J. Math. 221, 159-165 (2005). 